\title{
Hosing Instability of the Drive Electron Beam in the E157 Plasma-Wakefield Acceleration Experiment at the Stanford Linear Accelerator
}

By Brent Edward Blue 


\title{
UNIVERSITY OF CALIFORNIA
}

\author{
Los Angeles
}

\author{
Hosing Instability of the Drive Electron Beam in the \\ E157 Plasma-Wakefield Acceleration Experiment \\ at the Stanford Linear Accelerator
}

\author{
A thesis submitted in partial satisfaction \\ of the requirements for the degree Master of Science \\ in Electrical Engineering
}

by

Brent Edward Blue 


\section{Contents}

List of Figures $\quad$ iv

Acknowledgements $\quad$ vi

Abstract viii

1 Introduction 1

1.1 What is the PWFA? $\quad$........................................ 1

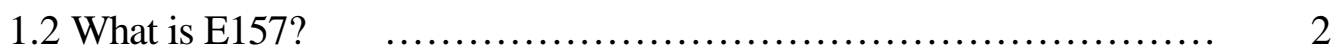

1.3 What is the Hosing Instability? $\quad$............................... 7

1.4 How Might One Look for the Hosing Instability? $\quad$............. 9

2 Theory of the Hosing Instability 11

2.1 Theory of the Hosing Instability - Offset Oscillations $\quad \ldots \ldots \ldots \ldots \ldots . \quad 11$

2.2 Theory of Hosing II $\quad$....................................... 14

2.3 Limitations to the Theory of Hosing $\quad$........................ 21

3 Experimental Setup $\quad \mathbf{2 3}$

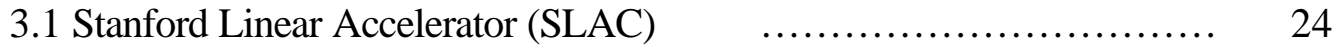

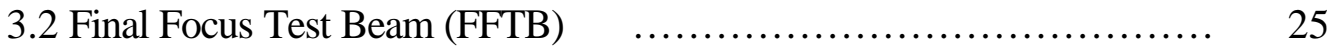

3.3 Lithium plasma source $\quad$........................................ 27

3.4 Optical Transition Radiators (OTR) $\quad$.......................... 28

3.5 Beam Position Monitors (BPMs) .......................... 32

3.6 Aerogel Cherenkov Radiator $\quad$.............................. 33

4 Results $\quad 37$

4.1 Initial Beam Condition $\quad$....................................... 37

4.2 Centroid Oscillations $\quad$..................................... 42

4.3 Tail Flipping $\quad$.............................................. 47

4.4 Tail Growth on Streak Camera Diagnostic $\quad$.................. 53

4.5 BPM Data for a High Density Run $\quad$........................ 56

5 Conclusion $\quad \mathbf{6 2}$

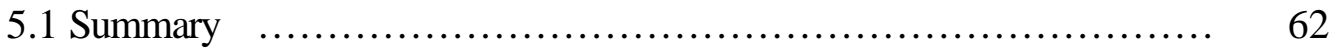

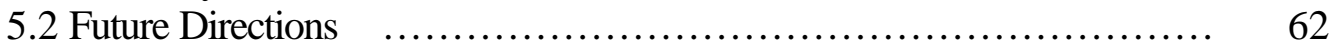

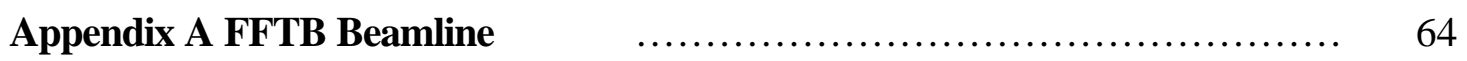

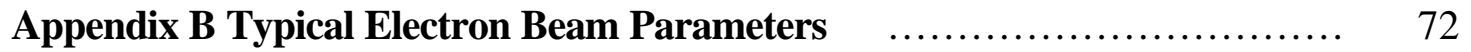

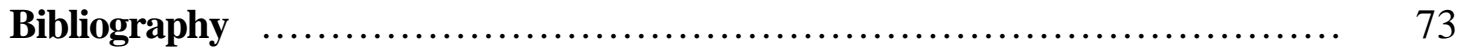




\section{List of Figures}

1.1.1 Cartoon of the electron beam displacing electrons and generating

a plasma wave

1.2.1 Energy vs. Year For High Energy Accelerators $\quad \ldots \ldots \ldots \ldots \ldots \ldots \ldots . . . \ldots$

1.2.2 Schematic for the NLC with SLAC drawn for size comparison $\quad \ldots \ldots . .5$

1.3.1 Cartoon of tilted beam before plasma (A), inside the plasma (B), and after the plasma $(\mathrm{C})$. The nomenclature for our tilted beam $(\mathrm{A})$ is the head defines the axis and the centroid is displaced from the axis by $\mathrm{r}_{\mathrm{o}}$. In the plasma, the head goes straight while the body oscillates. As the beam exists the plasma, the head continues straight while the body, with some perpendicular momentum, travels off at some angle.

2.2.1 Parameter Space of Growth Factor for an Offset Beam in a $140 \mathrm{~cm}$ long plasma

2.2.2 Parameter Space of Growth Factor for a Tilted Beam in a $140 \mathrm{~cm}$ long plasma

2.2.3 Oscillation of a tilted beam in a $2 \times 10^{14} \mathrm{~cm}^{-3}$ plasma for a channel which is formed $2 \mathrm{ps}$ before the centroid

2.2.4 Oscillation of a tilted beam at the end of a $140 \mathrm{~cm}$ long plasma for a channel which is formed 2ps before the centroid

2.3.1 Dynamics of Ion Channel Formation

3.0.1 Experimental Setup and Diagnostic Locations

3.2.1 Diagram of the FFTB near IP1 with relevant diagnostic locations marked .

3.3.1 Diagram of Lithium Oven Setup and Depiction of Lithium Column $\quad \ldots . . . \quad 28$

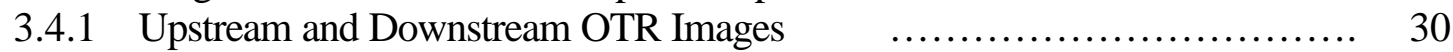

3.4.2 Image analysis of upstream and downstream OTR images............... 31

3.5.1 Typical Stripline BPM $\quad$................................... 32

3.6.1 Time Integrated Cherenkov Image depicting the parts of the beam seen by the horizontal and vertical slits on the streak camera

3.6.2 Typical Streak Camera image showing both the horizontal and vertical streaks $\quad$.............................................. 35

3.6.3 Diagram of Cherenkov radiation detector setup $\quad \ldots \ldots \ldots \ldots \ldots \ldots \ldots . . \ldots \ldots$

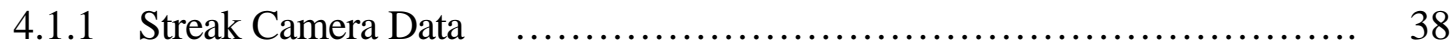

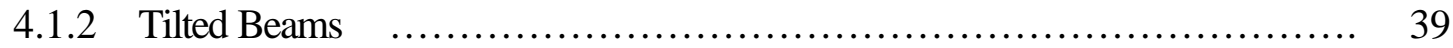

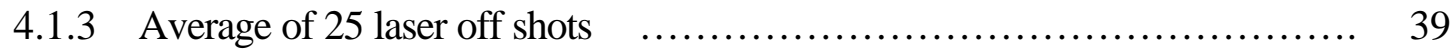




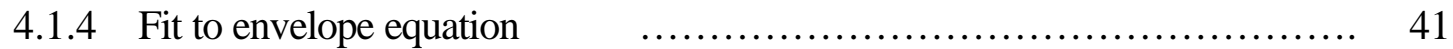

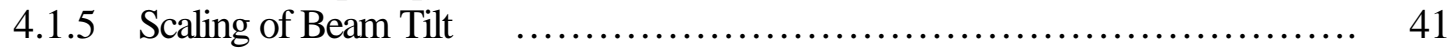

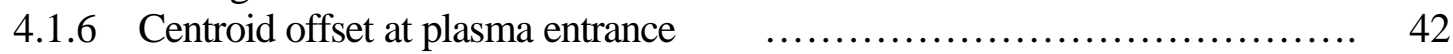

4.2.1 Upstream OTR images showing tails in $\mathrm{x}$-y plane $\quad \ldots \ldots \ldots \ldots \ldots \ldots \ldots \ldots . \ldots \ldots$

4.2.2 Centroid Oscillations downstream from the plasma in both

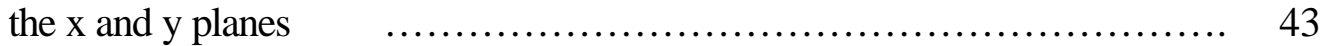

4.2.3 Centroid Oscillations on downstream OTR $\quad$........................... 44

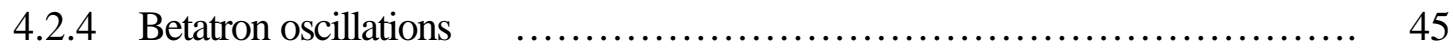

4.2.5 Single electron motion within the beam $\quad$............................ 46

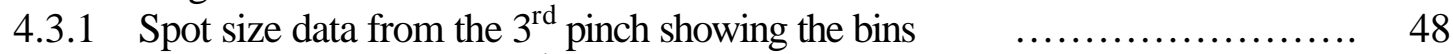

4.3.2 Spot size data from the $4^{\text {th }}$ pinch showing the bins $\quad \ldots \ldots \ldots \ldots \ldots \ldots \ldots \ldots \ldots . \ldots \ldots$

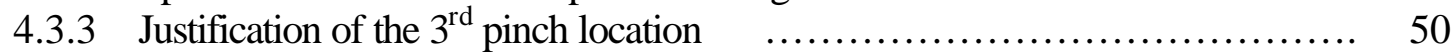

4.3.4 Correspondence between horizontal and vertical pinch energy at the $4^{\text {th }}$ pinch .................................................. 50

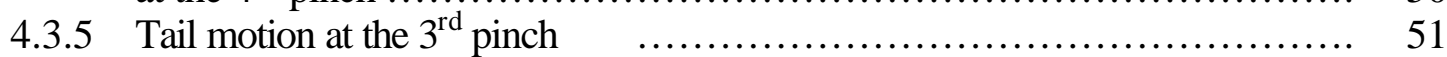

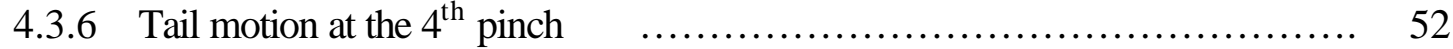

4.4.1 Time slice analysis at laser off, second, and fourth pinch $\quad \ldots \ldots \ldots \ldots \ldots . \quad 54$

4.4.2 Growth of normalized slice offsets vs. theoretical hosing curve $\quad \ldots \ldots . \quad 55$

4.5.1 Oscillations of beam centroid after plasma exit as measured on downstream OTR and on BPMs 6130, 6160, 6167, and $6170 \quad \ldots \ldots . \quad 57$

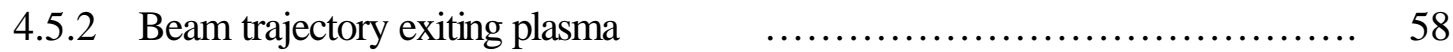

4.5.3 Slope of the beams exit trajectory as a function of laser energy $\quad \ldots \ldots . \quad 58$

4.5.4 Plot of envelope equation (shown in blue) versus plasma density superimposed on a plot of the beams spot size (red diamonds) versus incident UV laser energy. This fit is used to convert the incident UV laser energy into plasma density. $\quad$.................... $\quad 59$

4.5.5 Perpendicular Energy of Beam in a High Density Plasma $\quad \ldots \ldots \ldots \ldots \ldots . . .61$ 


\section{Acknowledgements}

It has been a great pleasure to work in the fields of plasma physics at UCLA and USC and the field of high-energy physics at Stanford. I would like to thank all of the faculty, staff, and students that I have worked and collaborated with at these first-rate institutions.

In particular I would like to first thank Dean Katsouleas for his support and guidance. This work would not have been possible if it were not for the many long hours in the E157 trailer Tom spent teaching me the dynamics of an electron beam in a plasma. I would like to also thank Prof. Muggli for not only working on the E157 collaboration, but also for his guidance on plasma physics in general.

I would like to also thank my colleagues at Stanford. Prof. Siemann has helped me out considerably in the fields of high-energy physics. Special thanks needs to be given for all of the Matlab code he has written which made my data analysis possible. I am eternally grateful to all of the work Dr. Hogan has contributed to the El57 experiment. If it were not for his work, none of my work would have been possible.

Special thanks need to be given to my colleagues at UCLA. Dr. Chris Clayton has taught me about physics and opened my eyes on seeing problems in a whole new light. 
Ken Marsh deserves special credit for bringing me on to the E157 collaboration. I would also like to thank Sho Wang for his "can-do-it" attitude and his support during the many hours we spent working in the trailer.

Finally, I would like to thank Prof. Chan Joshi, my advisor, who has been working with me on every detail of this research. If it were not for his guidance and support, this thesis would not be possible. 


\author{
ABSTRACT OF THE THESIS \\ Hosing Instability of the Drive Electron Beam in the \\ E157 Plasma-Wakefield Acceleration Experiment \\ at the Stanford Linear Accelerator \\ by \\ Brent Edward Blue

\begin{abstract}
Master of Science in Electrical Engineering
University of California, Los Angeles, 2000

Professor Chandrasekhar J. Joshi, Chair
\end{abstract}

In the plasma-wakefield experiment at SLAC, known as E157, an ultra-relativistic electron beam is used to both excite and witness a plasma wave for advanced accelerator applications. If the beam is tilted, then it will undergo transverse oscillations inside of the plasma. These oscillations can grow exponentially via an instability know as the electron hose instability. The linear theory of electron-hose instability in a uniform ion column predicts that for the parameters of the E157 experiment (beam charge, bunch length, and plasma density) a growth of the centroid offset should occur. Analysis of the E157 data has provided four critical results. The 
first was that the incoming beam did have a tilt. The tilt was much smaller than the radius and was measured to be $5.3 \mu \mathrm{m} / \sigma_{\mathrm{z}}$ at the entrance of the plasma (IP1.) The second was the beam centroid oscillates in the ion channel at half the frequency of the beam radius (betatron beam oscillations), and these oscillations can be predicted by the envelope equation. Third, up to the maximum operating plasma density of E157 $\left(\sim 2 \times 10^{14} \mathrm{~cm}^{-3}\right)$, no growth of the centroid offset was measured. Finally, time-resolved data of the beam shows that up to this density, no significant growth of the tail of the beam (up to 8ps from the centroid) occurred even though the beam had an initial tilt. 


\section{Introduction}

\subsection{What is the PWFA?}

Plasma based accelerators are of great interest because of their potential to accelerate charged particles at high gradients. Conventional radio frequency accelerators are limited to approximately $100 \mathrm{MV} / \mathrm{m}$. This limit is partly due to breakdown in the walls of the structure. On the other hand, a plasma can sustain electron plasma waves with electric fields on the order of the nonrelativistic wave breaking field [1], $E_{o}=c m_{e} \omega_{p} / e$, where $\omega_{p}=\left(4 \pi n_{o} e^{2} / m_{e}\right)^{1 / 2}$ is the electron plasma frequency and $n_{o}$ is the electron plasma density. Since the electric field scales as the square root of density, electric fields are on the order of 1-100 $\mathrm{GV} / \mathrm{m}$ for plasma densities in the range of $10^{14}-10^{18} \mathrm{~cm}^{-3}$.

Plasma based accelerators utilize a laser or a charged particle beam to drive a relativistically propagating plasma wave [2]. Laser driven accelerator schemes include the beat-wave accelerator [3], the laser-wake field accelerator [4], and the selfmodulated wake field accelerator [5]. These schemes possess the ability to generate high density plasma waves $\left(\sim 10^{18} \mathrm{~cm}^{-3}\right)$, and therefore, high electric field gradients $(\sim 100 \mathrm{GV} / \mathrm{m}$.) The shortcoming of these schemes is that the length of the accelerator is limited to a few Rayleigh lengths of the laser beam $(<1 \mathrm{~cm}$. $)$ Charged particle beam driven plasma wake field accelerators (PWFA) are capable of long interaction 
lengths (on the order of meters), at the expense of a decreased accelerating gradient ( $10-1000 \mathrm{MV} / \mathrm{m}$.)

In the PWFA, the space-charge force of the drive beam excites plasma waves (figure 1.1.1). For the case of an electron drive beam, the plasma electrons will be expelled radially. If the beam density $n$ is greater than the plasma density $n$ ("blowout" regime), then all plasma electrons will be expelled, leaving behind an ion column. After passage of the beam, the ion column space charge will pull back the expelled electrons. These electrons rush back in to the ion column and create a large density spike. The strong fields in front of the density spike are used to accelerate the electron beam.

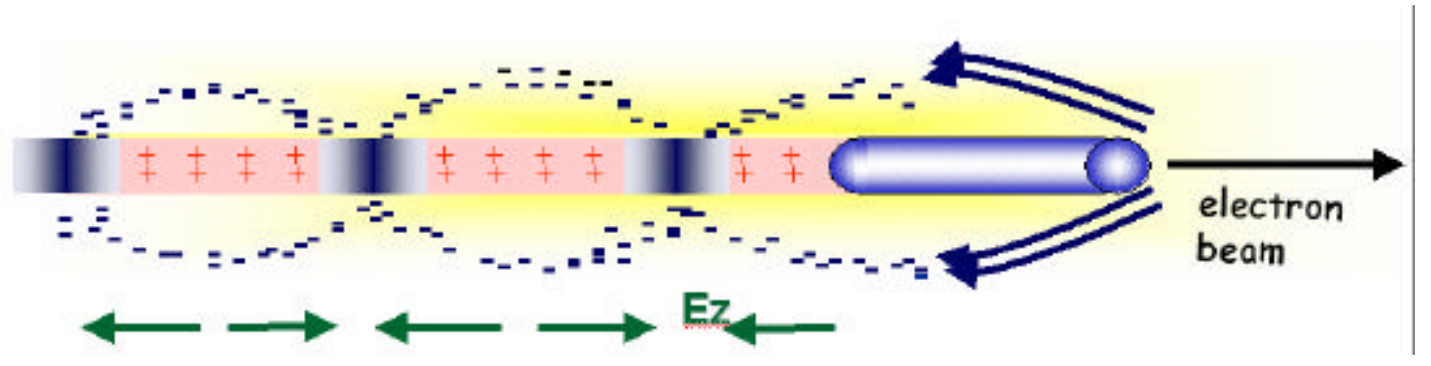

Figure 1.1.1 Cartoon of the electron beam displacing electrons and generating a plasma wave

\subsection{What is E157?}

E157 was an experiment to study acceleration of $30 \mathrm{GeV}$ electrons over 1.4 meters in a plasma wakefield accelerator [6]. The experiment was run at the Stanford Linear Accelerator (SLAC.) It was a collaboration between the University of 
California - Los Angeles, the University of Southern California, Lawrence Berkeley National Laboratory, and Stanford University. The goal of the experiment was to explore and develop techniques needed to implement a high-gradient PWFA in a large-scale accelerator. With respect to the electron beam, the goals were to study the longitudinal effects (acceleration of the tail and deceleration of the beam centroid) and the transverse effects (betatron oscillations, emission of betatron radiation, and hosing).

The motivation for this experiment is to not only explore new physics, but to also investigate technology for future high-energy colliders. High-energy colliders are used to study the fundamental building block of nature. The higher the energy of the beams, the more closely we can probe the structure of matter. The highest obtained collision energy was $104.5 \mathrm{GeV}$ electrons on $104.5 \mathrm{GeV}$ positrons in the Large Electron Positron Collider (LEP) at the European Organization for Nuclear Research (CERN.) At SLAC, the collision energies are on the order of $50 \mathrm{GeV}$ on $50 \mathrm{CeV}$. The next generation accelerators are designed to be $500 \mathrm{GeV}$ on $500 \mathrm{GeV}$. One design in development is the Next Linear Collider (NLC) [7]. The NLC is over $30 \mathrm{~km}$ long (SLAC is $4 \mathrm{~km}$ long, see figure 1.2.2) since it relies on conventional RF accelerator technology. Clearly new technology must be developed which increases accelerating gradients into the $\mathrm{GeV}$ per meter range. Beam driven plasma wakefield accelerators are the most attractive advanced accelerator concept because they can support $\mathrm{GeV} / \mathrm{m}$ accelerating gradients over meter lengths. 


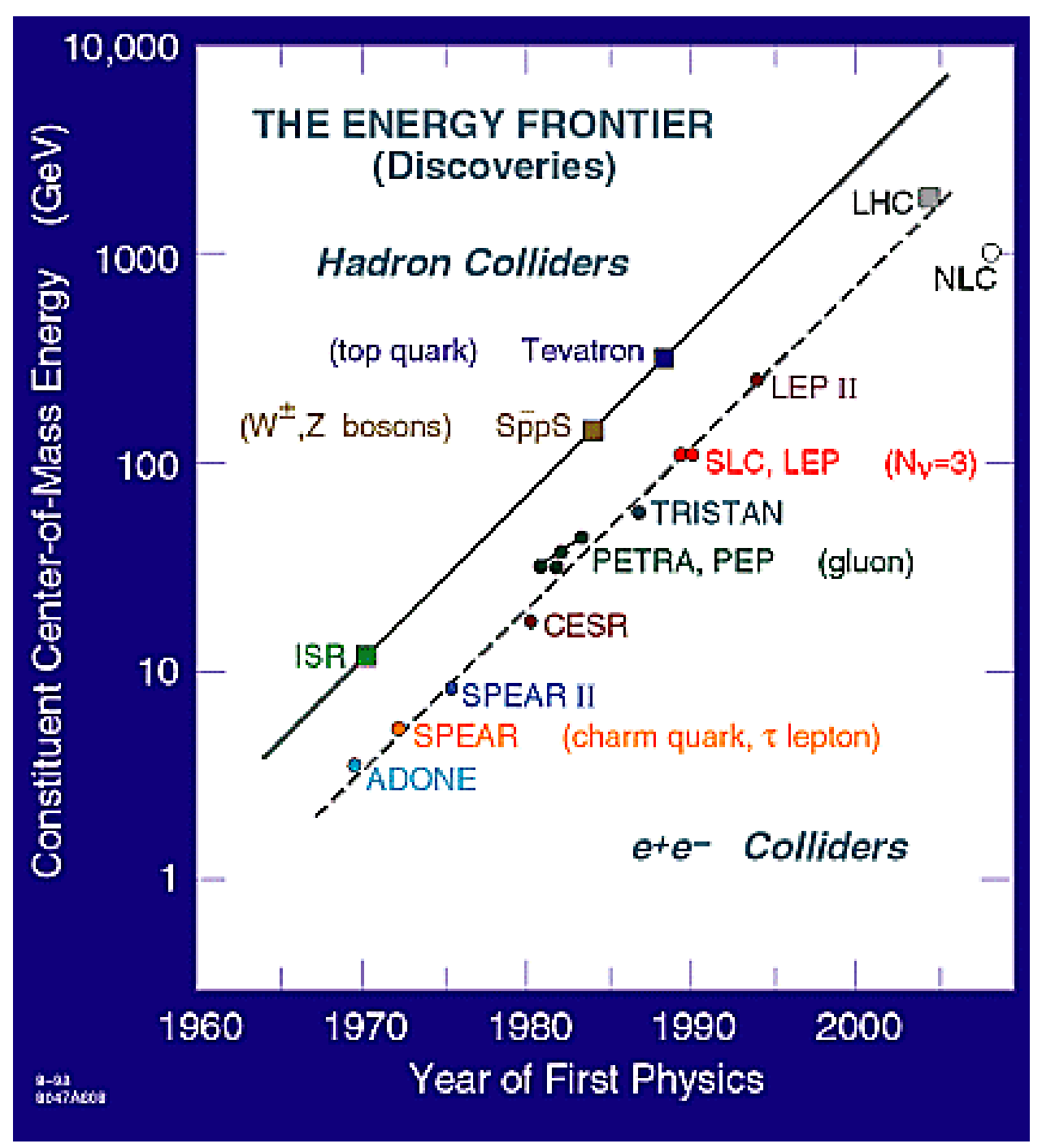

Figure 1.2.1 Energy vs. Year For High Energy Accelerators 


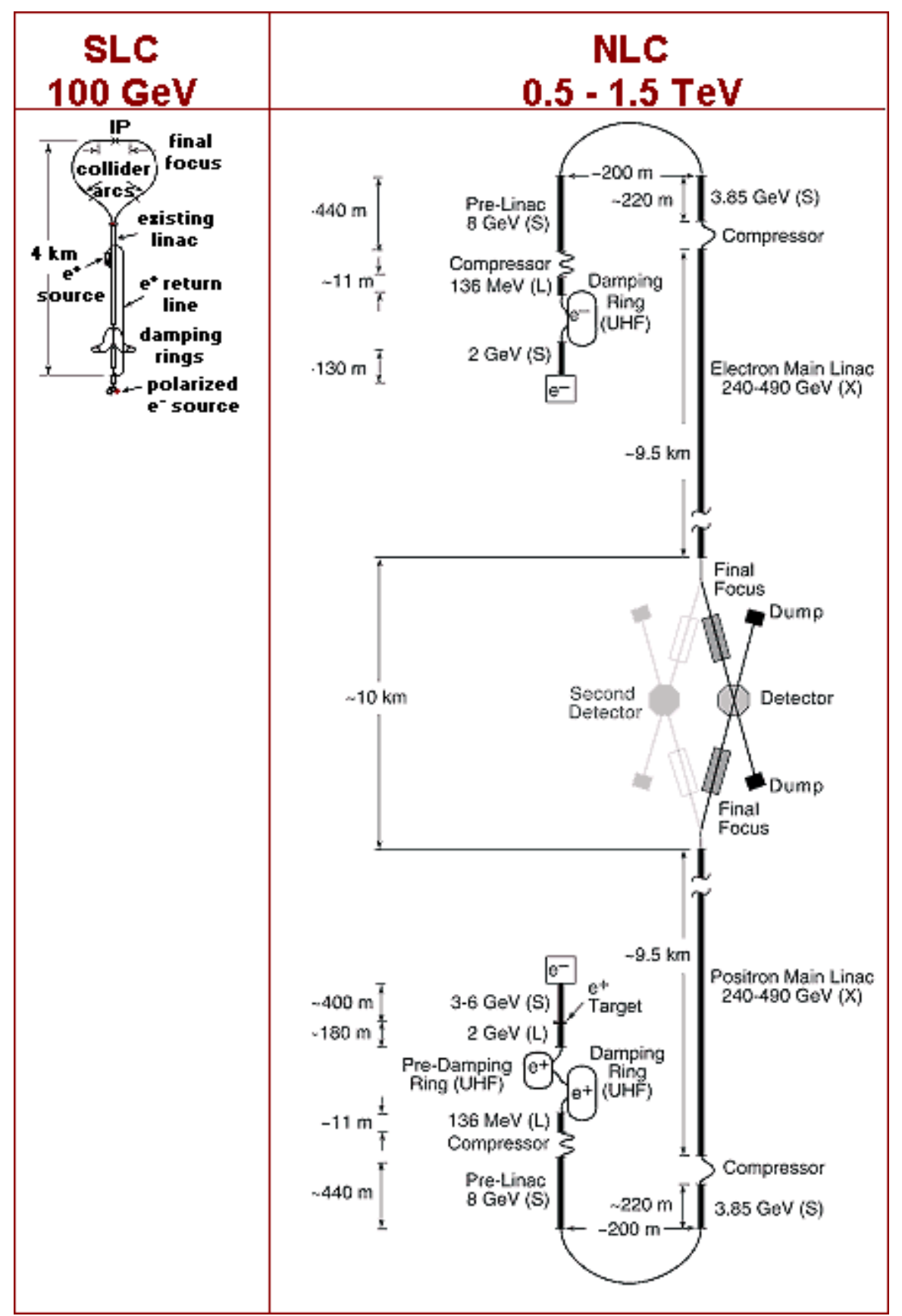

Figure 1.2.2 Schematic for the NLC with SLAC drawn for size comparison 
The basic idea of the E157 experiment is to use a single electron bunch where the front of the beam excites a plasma wave and the tail of the beam witnesses the resulting accelerating field. The nominal beam parameters were $2 \times 10^{10}, 30 \mathrm{GeV}$ electrons, a bunch length of $0.7 \mathrm{~mm}$, and a transverse spot size of $40 \mu \mathrm{m}$. This beam was propagated through a $1.4 \mathrm{~m}$ long Lithium plasma of a density up to $2 \times 10^{14} \mathrm{e}^{-} / \mathrm{cm}^{-3}$. The plasma source is positioned at interaction point 1 in the Final Focus Test Beam (FFTB) at SLAC. The FFTB is a straight shot down the $3 \mathrm{~km}$ long accelerator and was designed to investigate the factors that limit the size and stability of the beam at the collision point of a linear collider. The importance of the FFTB design to our experiment is that the beam optics and diagnostics are capable of delivering a high quality beam to our experiment. In addition to the diagnostics built into the FFTP (beam position monitors, current measuring torroids, and wire scanners), the experiment required single shot beam profile measurements before and after the plasma and time resolved measurements after an energy dispersive bend magnet. These diagnostics allowed the E157 collaboration to study the transverse and longitudinal dynamics of a high peak current (>100A), ultra-relativistic electron bunch in a $1.4 \mathrm{~m}$ of $0-2 \times 10^{14} \mathrm{~cm}^{-3}$ underdense plasma. 


\subsection{What is the hosing instability?}

Propagation of intense charged beams in an under-dense plasma (also known as the ion-focused regime, IFR) has drawn considerable interest from both the accelerator and radiation research communities. Some of the accelerator applications are, in addition to the PWFA, the plasma lens [8], the continuous plasma focus [9], and the plasma emittance damper [10]. In addition, both theoretical [11] and experimental work [12] have looked at the generation of coherent radiation from beams in the IFR. Both theoretical [13] and experimental [14] work have shown that a transverse instability exists.

These transverse instabilities arise when a non-uniform transverse force acts upon the beam. Such a situation can occur when an electron beam is offset in a uniform ion column. The term hose instability is used whenever a flexible confinement system is used. Hose instabilities were first observed with long pulse electron beams $\left(\tau_{b} \geq 1 \mu s\right.$.) Stable beam transport was shown to be disrupted by the ion-hose instability [15]. For such long pulses, the ions are mobile and a feedback occurs between the displaced electrons and ions. If the electron pulse length is short enough so that the ions can be considered immobile, another hose instability is predicted to occur, the electron hose instability. In this instability the relativistic electron beam exhibits a transverse instability due to the coupling of the beam centroid to the plasma electrons at the ion-channel edge. 


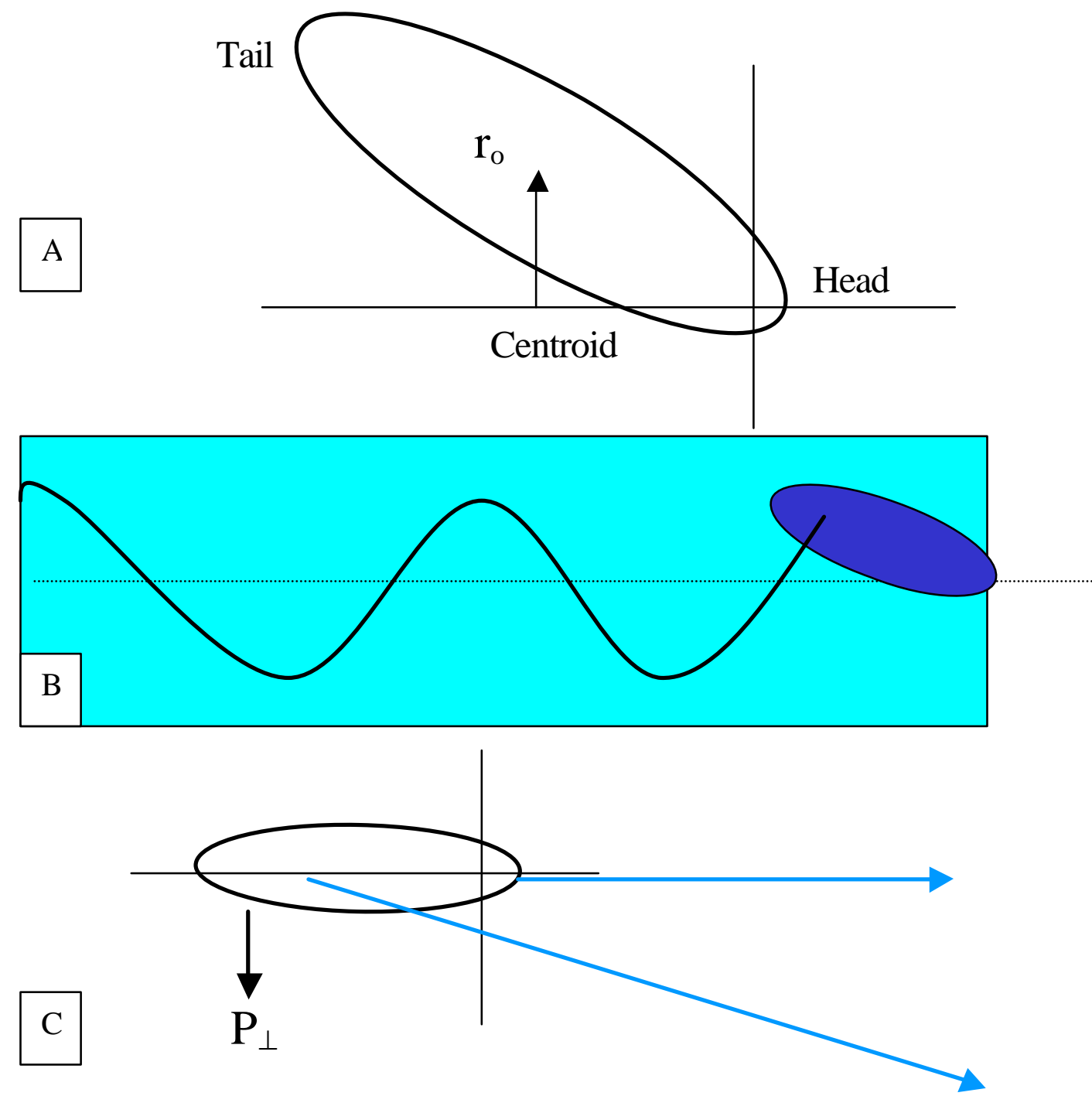

Figure 1.3.1 Cartoon of tilted beam before plasma (A), inside the plasma (B), and after the plasma $(\mathrm{C})$. The nomenclature for our tilted beam (A) is the head defines the axis and the centroid is displaced from the axis by $\mathfrak{b}$. In the plasma, the head goes straight while the body oscillates. As the beam exists the plasma, the head continues straight while the body, with some perpendicular momentum, travels off at some angle. 
Growth rates for he electron hose instability have been calculated based on a simplified model [16]. For the E157 beam parameters, the growth rate of the instability was predicted to be very rapid and therefore deleterious to the experiment. The beam centroid offset was predicted to witness an amplification of $10^{0}-10^{2} \mathrm{x}[17]$. If these predictions were correct, the electron hose instability would not only increase the difficulty of interpreting our experimental results, but it would also hinder the use of PWFAs in future accelerators.

\subsection{How might one look for the hose instability?}

In the E157 experiment, the head of the beam blows out the plasma electrons to form the ion column. If the beam enters the plasma with a tilt, then the centroid of the beam will be offset in the ion column. If the electron hose instability exists, then this offset will grow inside of the plasma. There exist two diagnostics that can resolve the effects of the electron hose instability. They are the beam position monitors (BPMs) and the streak camera. The BPMs will track the location of the beam centroid, while the streak camera will temporally resolve the relative position of each beam segment. Each diagnostic has its own advantages and disadvantages.

The BPMs track the position of the beam centroid as it drifts out of the plasma. In order to look for hosing using this method, an energy scan data run must be taken. An energy scan data run is when the plasma density is varied from 0 to $2 \times 10^{14} \mathrm{~cm}^{-3}$ over 200 shots by varying the laser energy needed to ionize the Lithium vapor. By 
plotting the centroid displacement as a function of distance after the plasma, the trajectory of the centroid exiting the plasma can be calculated for each shot. Next you plot the slope of the centroid's trajectory vs. plasma density. If a hosing instability exists, the slope of the trajectory will increase nonlinearly with plasma density.

The streak camera can be used to measure the head-tail offset for every shot. If the laser is turned off (i.e. no plasma) then the initial tilt on the beam can be measured. With the plasma turned on, the resultant beam tilt can be measured. This will allow us to analyze how the plasma affects the entire beam, not just the centroid. One difficulty with this measurement is keeping the beam aligned to the slit of the streak camera. If the plasma imparts a large deflection to the beam, it can move the image off of the slit.

One limitation of the current experimental setup is that the streak camera diagnostic and four (out of five) BPMs are located after a large bending magnet. This large bending magnet adds dispersion to the vertical plane that is used for the experiment's energy gain diagnostic. However, this magnet hampers the hosing diagnostics because the analysis requires a pure drift space after the plasma. This limits the analysis to the horizontal plane (x-axis) which does not gain dispersion through the bending magnet. Results will show that a tail is in the $x y$ plane (section 4.1), but only the horizontal component of the tail will be quantified. 


\section{Theory of the Hosing Instability}

\subsection{Theory of the hose instability - Offset Oscillations.}

The first step towards looking for the hosing instability is to calculate the transverse motion of the beam in the absence of hosing. We will look at the special case of a relativistic electron beam confined by an ion channel. The initial conditions will be an electron beam and ion column with equal radii $r_{o}$ and uniform densities. The electron beam density is denoted by $n_{b}$ and the ion column density is

$$
\mathrm{n}_{\mathrm{p}}=f \mathrm{n}_{\mathrm{b}}
$$

The neutralization fraction, $f$, is less than unity and it accounts for the under-dense condition of the plasma density. We will assume that the ions are infinitely massive (i.e. they are rigid) and that the ion column is centered on the z-axis. The beam displacement, $\mathrm{y}(\mathrm{z}, \mathrm{t})$, will have an initial displacement

$$
\mathrm{y}_{\mathrm{o}}=\mathrm{y}(0, \mathrm{t})
$$

In the limit of small displacement, $\left|\mathrm{y}_{\mathrm{o}}\right| \ll \mathrm{r}_{0}$, the electric field of the displaced charge cylinders in the area region occupied by the cylinders is uniform with a magnitude linearly proportional to the displacement. The electric field is given by

$$
E_{y}(z, t)=-4 p \text { e } n_{b} y(z, t)
$$

This gives rise to a restoring force that is given by

$$
F_{y}(z, t)=4 p e^{2} n_{b} y(z, t)
$$


In addition to the force between the beam and the ion column, the beam position can also change by convection. The net change in the transverse velocity of the beam is

$$
\frac{\partial v_{y}}{\partial \tau}=-\beta c \frac{\partial v_{y}}{\partial z}-\left(\frac{4 \pi e^{2} n_{p}}{\gamma m_{e}}\right) y
$$

Rearranging terms and defining the convective derivative and beam plasma frequency

$$
\begin{gathered}
\frac{D}{D t}=\frac{\partial}{\partial \tau}+\beta c \frac{\partial}{\partial z} \\
\omega_{b}=\left(\frac{4 \pi e^{2} n_{b}}{\gamma m_{e}}\right)^{1 / 2}
\end{gathered}
$$

we get

$$
\frac{D v_{y}}{D t}=-\left(f \omega_{\mathrm{b}}^{2}\right) y
$$

We can similarly derive an expression for the change in y at a point

$$
\frac{\delta y}{\delta t}=-\beta c \frac{\delta y}{\delta z}+v_{y}
$$

or

$$
\frac{D y}{D t}=\mathrm{v}_{y}
$$

Combining equations (2.1.8) and (2.1.10) in order to solve for the displacement as a function of initial displacement gives

$$
\frac{D^{2} y}{D t^{2}}=-\left(f \omega_{\mathrm{b}}^{2}\right) y
$$


or

$$
\frac{\delta^{2} y}{\delta t^{2}}+2 \beta c \frac{\delta}{\delta t} \frac{\delta y}{\delta z}+\beta^{2} c^{2} \frac{\delta^{2} y}{\delta z^{2}}=-\left(f \omega_{\mathrm{b}}^{2}\right) y
$$

Calculating a steady state solution by setting the time derivative to zero yields

$$
\frac{\delta^{2} y}{\delta z^{2}}=-f\left(\frac{\omega_{b}}{\beta c}\right)^{2} y
$$

The solution to this equation is

$$
y=y_{o} \cos \left(k_{b} z\right)
$$

where

$$
k_{b}=\sqrt{f}\left(\omega_{b} / \beta c\right)
$$

This shows that if the electron beam enters the plasma with an offset, it will oscillate harmonically about the center of the ion channel.

The plasma column in the E157 experiment, L, was $140 \mathrm{~cm}$ long. The head of the beam blows out the electrons and defines the plasma channel. If the beam has a longitudinal-transverse correlation (i.e. tails or tilt), then the beam centroid is displaced from the center of the plasma column. If the beam enters the plasma with an offset of $y_{0}$, then it will exit the plasma with an offset of

$$
y_{L}=y_{0} \cos \left(k_{b} L\right)
$$


If $k_{b} L$ does not equal an integer number of pi, then the centroid will gain some perpendicular momentum as it exists the plasma. The slope of the centroid's exit trajectory is given by

$$
\dot{y}_{L}=y_{0} k_{b} \sin \left(k_{b} L\right)
$$

If an instability were present, we would expect to see a growth term in equation (2.1.14).

$$
y=y_{o} e^{A} \cos \left(k_{b} z\right)
$$

The basic form of the solution is correct, but now we will go into a more rigorous derivation for electron hosing.

\subsection{Theory of Hosing II [16]}

We will consider the relativistic beam propagation in an infinite, unmagnitized, preionized, and uniform plasma of density $n_{p}$. The plasma is under-dense in relation to the beam density, $n_{p}<n_{b}$. We assume that the Budker condition, $n_{p}>>n_{b} / ?^{2}$ is satisfied. This implies that all beam electrons will undergo transverse oscillations at a single "betatron frequency", $\omega_{\beta}=\omega_{p} /(2 \gamma)^{1 / 2}$. We will further assume a collisionless plasma and that the plasma skin depth, c/? , is much larger than the channel radius. By using the "rigid beam" model and the "frozen field" approximation, we find that the system response to a small perturbation, $?_{\mathrm{x}}(\mathrm{s}, \mathrm{t})$, is described by a beam breakup equation, where $\mathrm{s}$ is the longitudinal position of the beam in the laboratory frame and $\mathrm{t}$ 
can be thought of as an index that labels a given slice of the beam (i.e. the head of the beam is $\mathrm{t}=0 \mathrm{ps}$, and $\mathrm{t}=4 \mathrm{ps}$ refers to the beam slice $4 \mathrm{ps}$ behind the head).

$$
\left(\frac{\delta}{\delta s} \gamma \frac{\delta}{\delta s}+\gamma k_{\beta}^{2}\right) \xi_{x}(s, \tau)=\int_{0}^{\tau} W\left(\tau-\tau^{\prime}\right) \xi\left(s, \tau^{\prime}\right) \delta \tau^{\prime}
$$

where the wakefield, $\mathrm{W}$, is given by

$$
W(\tau)=\frac{\omega_{o}^{3}}{c^{2}} \sin \left(\omega_{o} \tau\right)
$$

$\mathrm{k}_{\beta}$ is the betatron wave number and $?_{\mathrm{o}}=?_{\mathrm{p}} /(2)^{1 / 2}$. An asymptotic form for the solution of equation (2.2.1) in the limit of strong focusing and a short bunch (i.e. picosecond pulses) is obtained by the method of steepest descents [18]

$$
\xi(s, \tau) \approx 0.263 \frac{\xi_{o}}{A^{1 / 2}} e^{A} \cos \left(k_{\beta} s-\frac{A}{\sqrt{3}}+\frac{\pi}{12}\right)
$$

where

$$
A(s, \tau)=\frac{3^{3 / 2}}{4}\left[\left(k_{\beta} s\right)\left(\omega_{o} \tau\right)^{2}\right]^{1 / 3}
$$

The solution in equation (2.2.3) is for the case when then entire beam is offset in the plasma column. If the beam is tilted, the solution becomes [18]

$$
\xi(s, \tau) \approx 0.341 \frac{\xi_{o}}{A^{3 / 2}} e^{A} \cos \left(k_{\beta} s-\frac{A}{\sqrt{3}}+\frac{\pi}{12}\right)
$$

Figures 2.2.1 and 2.2.2 show how the centroid offset grows for different plasma densities and how the instability grows for beam slices farther from the beam head (i.e. increasing t). Notice how the growth factor, $\xi(s=140 \mathrm{~cm}, \tau) / \xi_{o}$, is significantly 
reduced for the tilted case. Results from section 4.5 indicate that the beam centroid is about $2 \mathrm{ps}$ back from the point of channel formation. The electrons that were to be accelerated were about $46 \mathrm{ps}$ behind the beam centroid. Figure 2.2.3 shows how the centroid and tail oscillates $(\mathrm{t}=2,4$, and $6 \mathrm{ps})$ inside of the plasma column and figure 2.2.4 shows how the centroid and tail oscillations will look downstream of the plasma during a plasma density scan. 


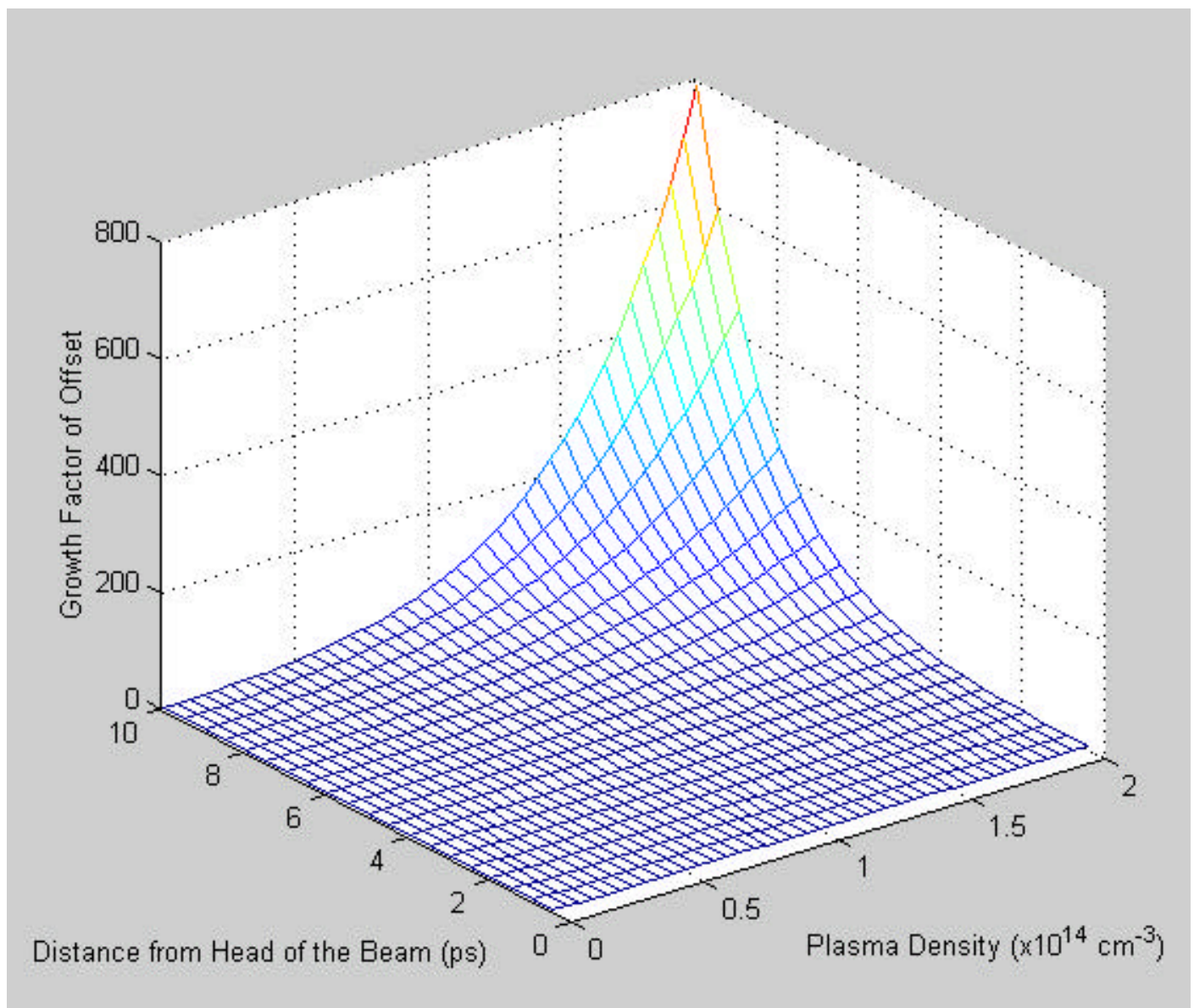

Figure 2.2.1 Parameter Space of Growth Factor for an Offset Beam in a $140 \mathrm{~cm}$ long plasma 


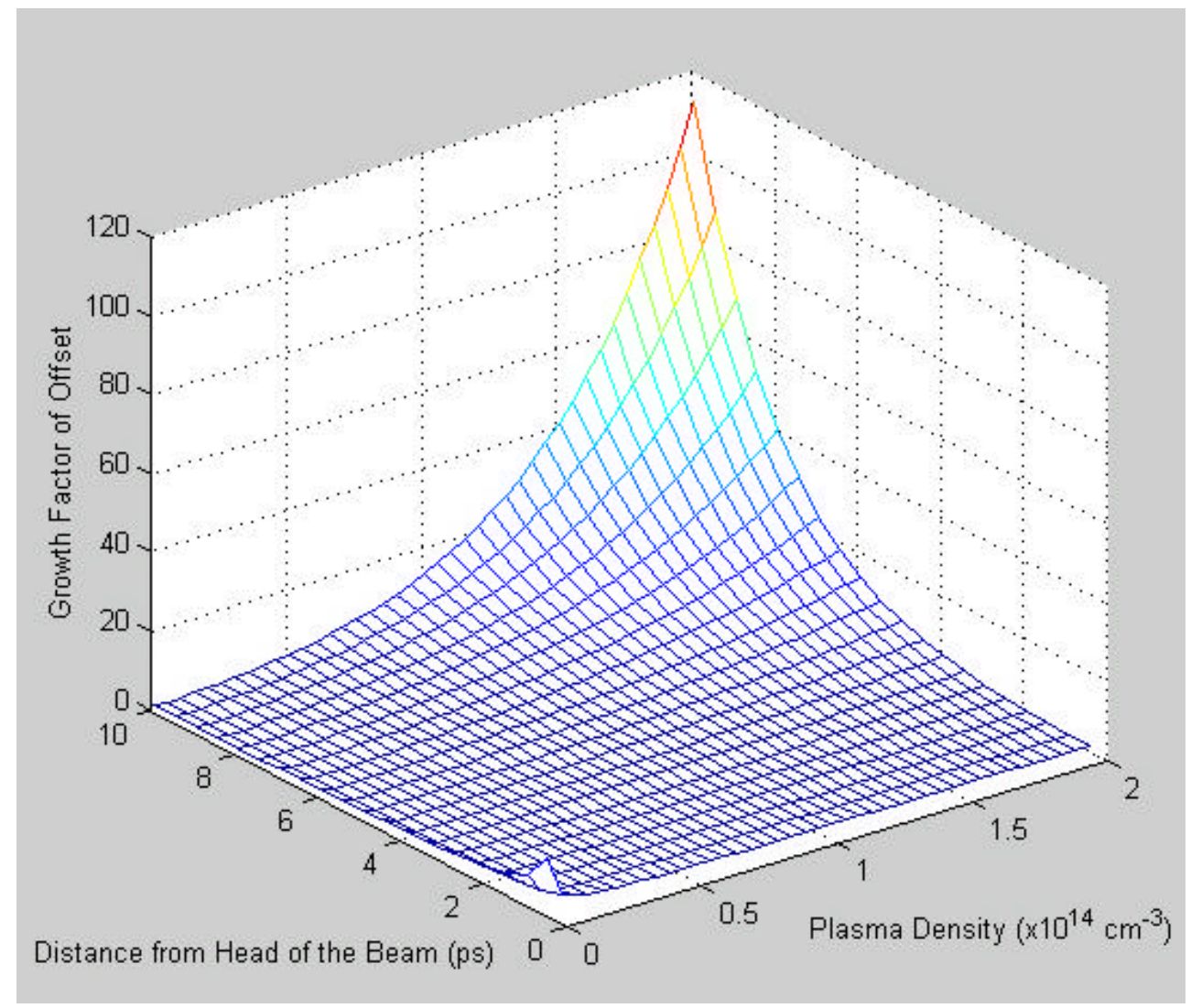

Figure 2.2.2 Parameter Space of Growth Factor for a Tilted Beam in

a $140 \mathrm{~cm}$ long plasma 


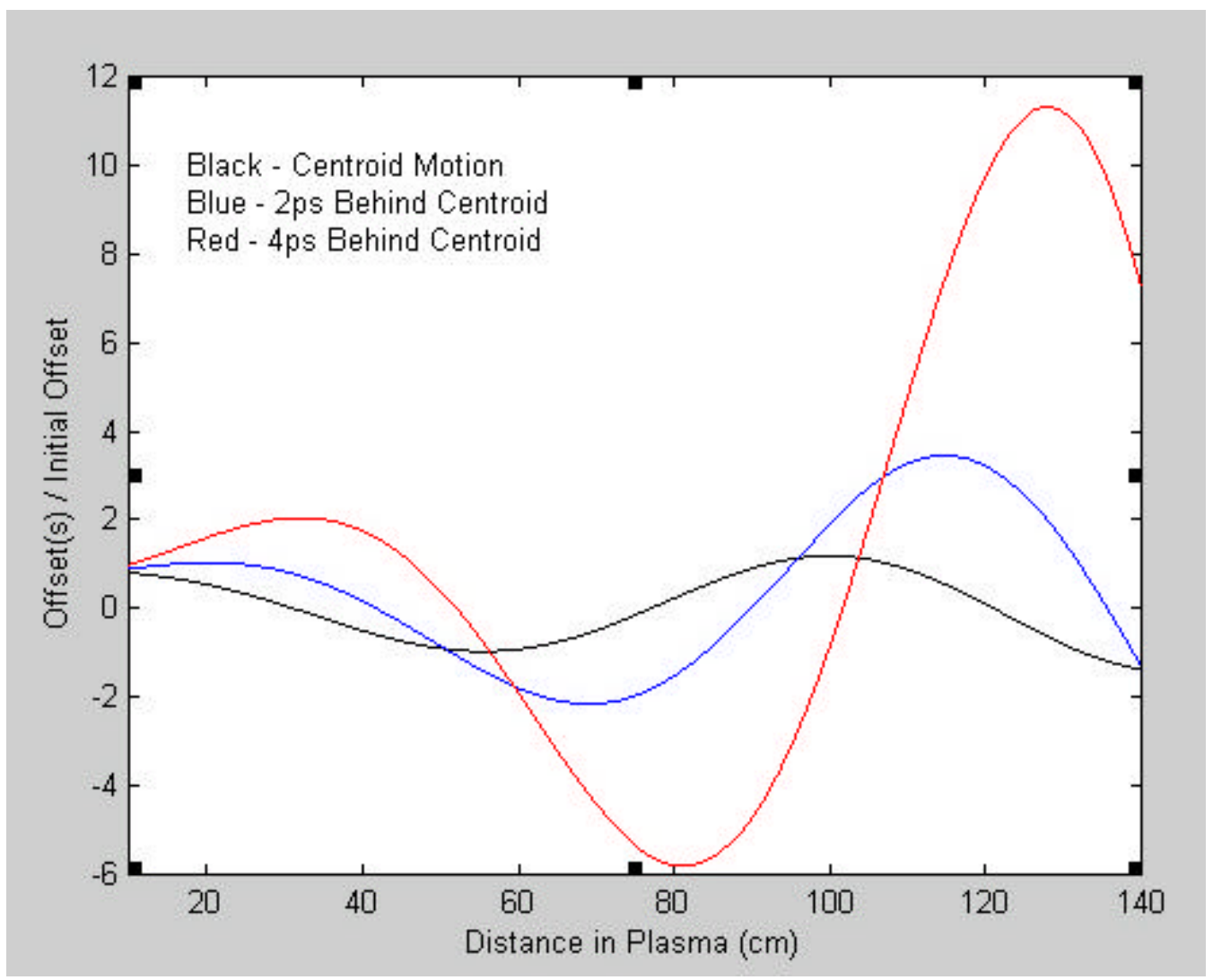

Figure 2.2.3 Oscillation of a tilted beam in a $2 \times 10^{14} \mathrm{~cm}^{-3}$ plasma for a channel which is formed $2 \mathrm{ps}$ before the centroid 


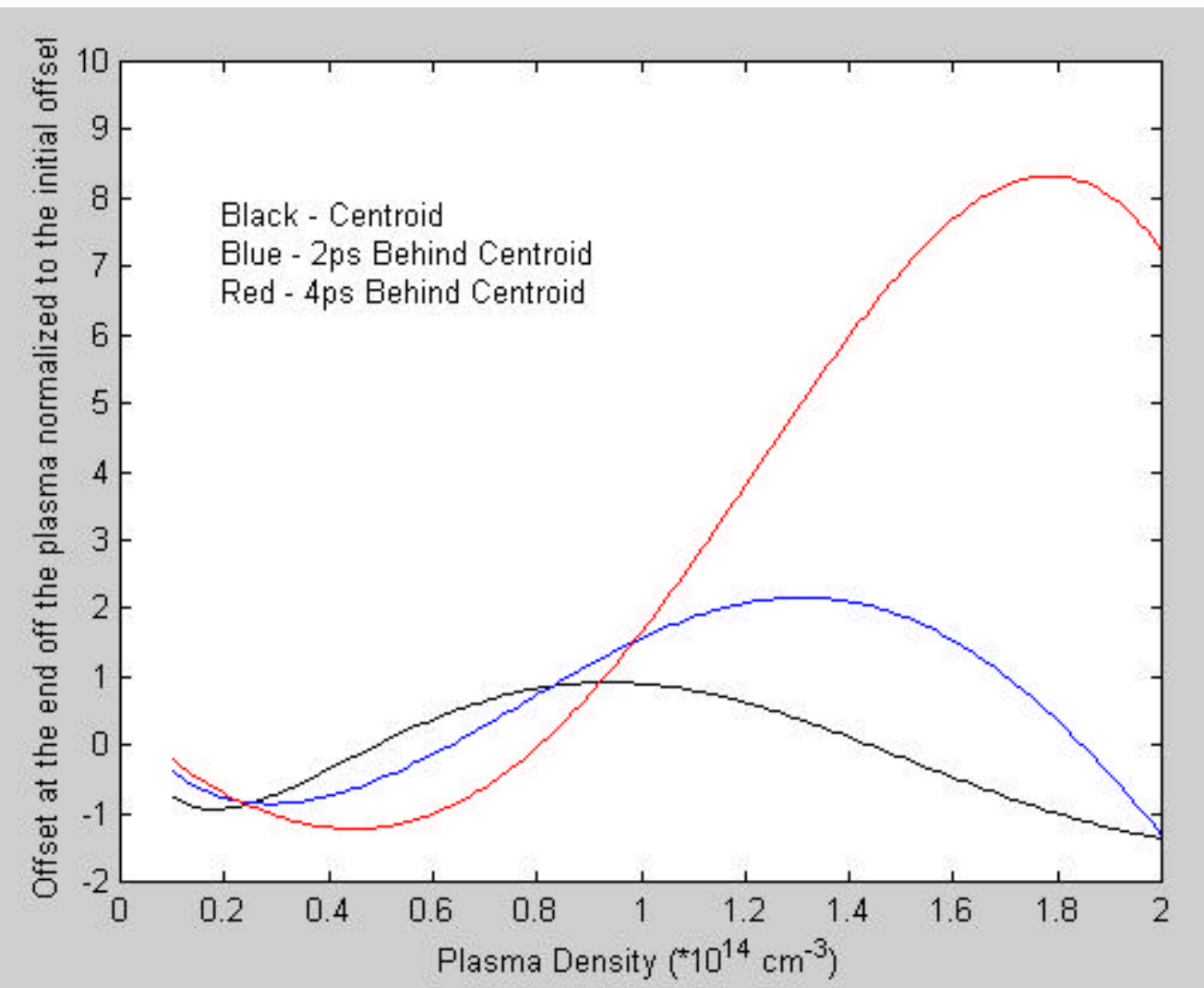

Figure 2.2.4 Oscillation of a tilted beam at the end of a $140 \mathrm{~cm}$ long plasma for a channel that is formed 2 ps before the centroid 


\subsection{Limitations to the Theory of Hosing}

In the E157 experiment, the beam propagates in an underdense plasma and both excites and witnesses the plasma wave. If two beams were used, one to drive the wave and a second to witness the acceleration, the second beam would be propagating in the ion-focused regime. The subtle difference is that a beam in under-dense plasma creates an ion channel, as opposed to the ion-focused regime that assumes a preformed ion channel. Although subtle, no hosing theory exists for the under-dense regime; all have assumed the ion-focused regime. In the past, this approximation was valid since the pulse length was greater than the plasma wavelength. In E157, the pulse length is on the order of the plasma wavelength, therefore the dynamics of the expelled electrons are important and the assumption of a preformed ion column is not a valid one.

Valuable insight can still be gained from the equations, but they need to be modified to take into account the formation of the ion column. A first approximation is to modify the beam position parameter, $\mathrm{t}$. $\mathrm{t}$ will no longer correspond to the distance from the head of the beam, rather the distance from channel formation. Since the channel can form near the beam centroid (figure 2.3.1) [19], the growth factor of hosing is significantly reduced (e.g. reduce $\mathrm{t}$ from $8 \mathrm{ps}$ to $2 \mathrm{ps}$ in figure 2.2.2). Therefore, one may not observe significant growth of the hosing instability in the centroid motion of the beam. However, the tail of the beam could still suffer a significant growth as seen in figures 2.2.3 and 2.2.4. 


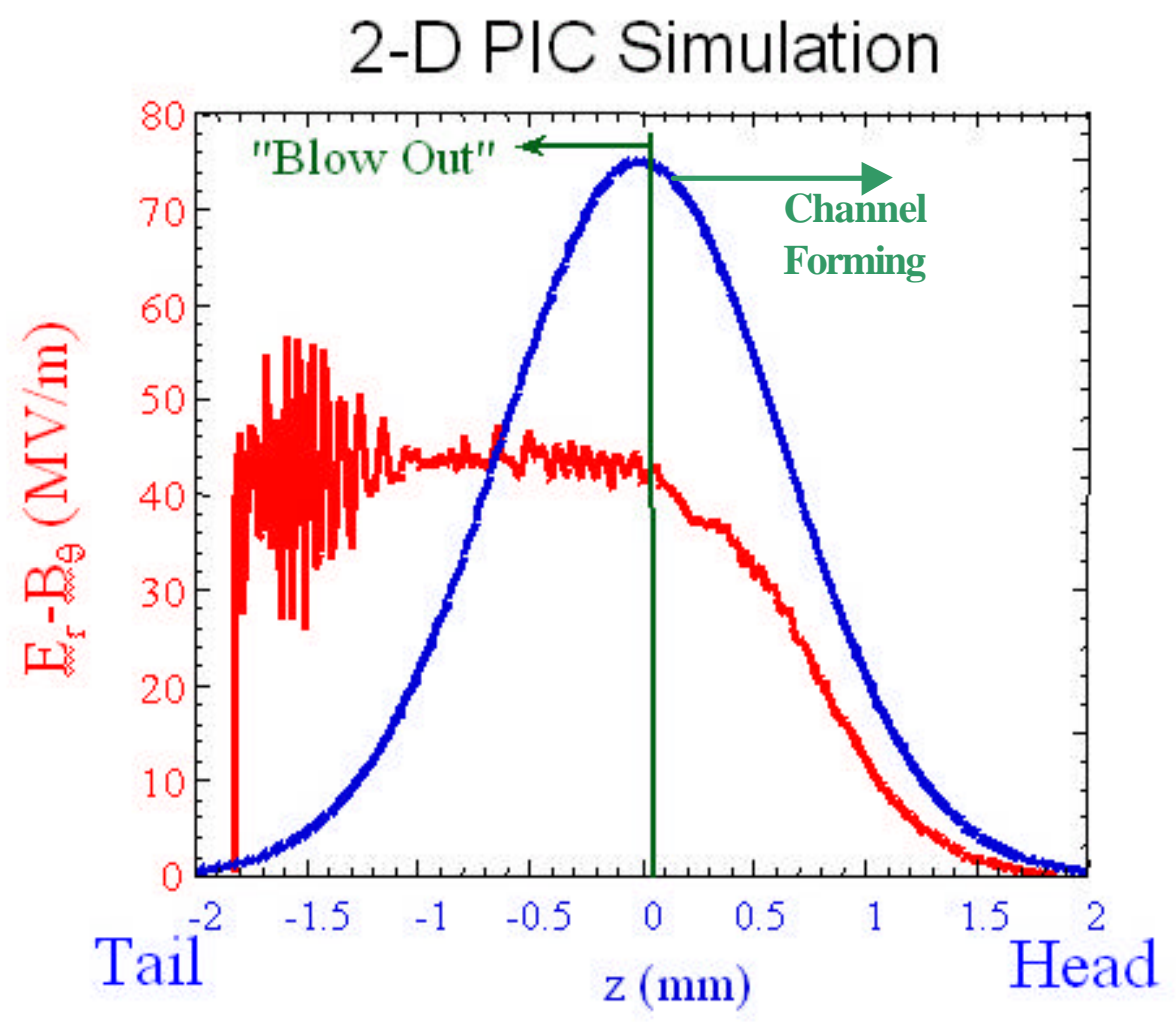

Figure 2.3.1 Dynamics of Ion Channel Formation. This is a 2-D Particle in Cell simulation of an electron beam (shown in blue) propagating in a plasma. The space charge of the beam expels plasma electrons radially to form an ion column. The radial electric field associated with the ion column is show in red. The magnitude of the field increases until all plasma electrons have been expelled and a pure ion column is left behind. At this point, known as "blow out", the magnitude of the radial field is constant. As one can see, the point of channel formation $(t=0)$ is not the head of the beam, rather it is located part way into the beam. 


\section{Experimental Setup}

The principle components of the experimental apparatus are the Lithium plasma source [20, 24], the optical transition radiators [21, 22, 24], the beam position monitors [23], and an aerogel Cherenkov radiator [24]. A brief description of each component will now be given. For a more detailed view of any specific component, refer to the references given above.

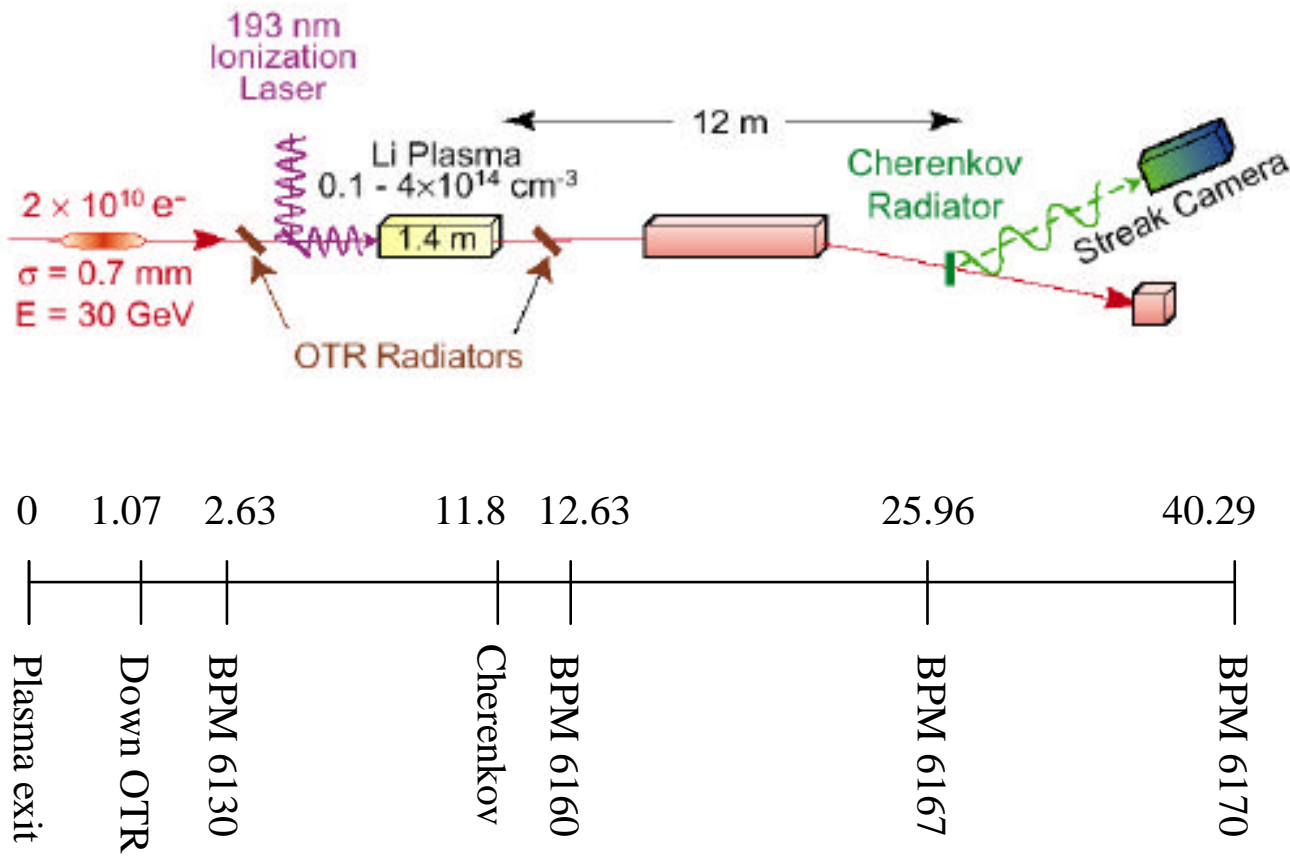

Figure 3.0.1 Experimental Setup and Diagnostic Locations 


\subsection{Stanford Linear Accelerator (SLAC)}

Before a description of the E157 experimental components is given, a brief introduction to SLAC is necessitated. SLAC is operated under contract from the United States Department of Energy (DOE) as a national basic research laboratory. Its function is to probe the structure of matter at the atomic scale with $x$-rays and at much smaller scales with electron and positron beams. The major facilities at SLAC are the linac, End Station A, SPEAR and SSRL, PEP II, SLC, and the FFTB. The linac is a three kilometer long accelerator capable of producing electron and positron beams with energies up to $50 \mathrm{GeV}$. End Station $\mathrm{A}$ is for fixed target experiments. Early work in End Station A showed that the constituents of the atomic nucleus, the proton and neutron, are themselves composed of smaller, more fundamental objects called quarks. The Stanford Synchrotron Radiation Laboratory (SSRL) uses the SPEAR storage ring to produce intense $\mathrm{x}$-ray and ultraviolet beams for probing matter on the atomic scale. PEP II is a storage ring for a B meson factory in which an experiment, BaBar, is seeking to answer why the universe is made of matter and not anti-matter. The Stanford Linear Collider (SLC) in conjunction with the Stanford Large Detector (SLD), analyzed collisions of $50 \mathrm{GeV}$ electrons on $50 \mathrm{GeV}$ positrons in order to determine the mass and other properties of the $Z^{0}$ particle, which is a carrier of the weak force of subatomic physics. The Final Focus Test Beam (FFTB) is a facility for research on future accelerator design. The E157 experiment is located inside the FFTB. 


\subsection{Final Focus Test Beam (FFTB)}

The FFTB was designed to be a facility to be used for the development and study of optical systems, instrumentation, and techniques needed to produce the small beam spot sizes required for future electron-positron colliders. The design consists of five key sections. The first part is a matching section to match the beam that appears at the end of the linac to the lattice of the FFTB beamline. This matching section also has lens to match the betatron space of the beam to the second section, the chromatic correction section. The second, third, and fourth sections are used to correct chromatic and geometric aberrations on the beam. The final section is a telescope that focus the beam down to a small spot size.

The optics of the FFTB consist of dipoles, quadrupoles, and sextapoles. In order to focus the beam down to nanometer spot sizes, the optics needed to be aligned to an accuracy on the order of a micron. The optics were mounted on 3-axis positioners that allowed the optics to be moved $\pm 1 \mathrm{~mm}$ with $300 \mathrm{~nm}$ resolution. To complement these optics, diagnostics were needed to determine the spot size and position of the beam along the beamline. These include torroids (to measure the beam's charge), wire scanners (to measure spot size), and BPMs (to measure the beam's posistion, Sec. 3.5). A diagram of the elements of the FFTB near interaction point 1 is given in figure 3.2.1 while a full diagram of the FFTB elements is given in appendix A. 


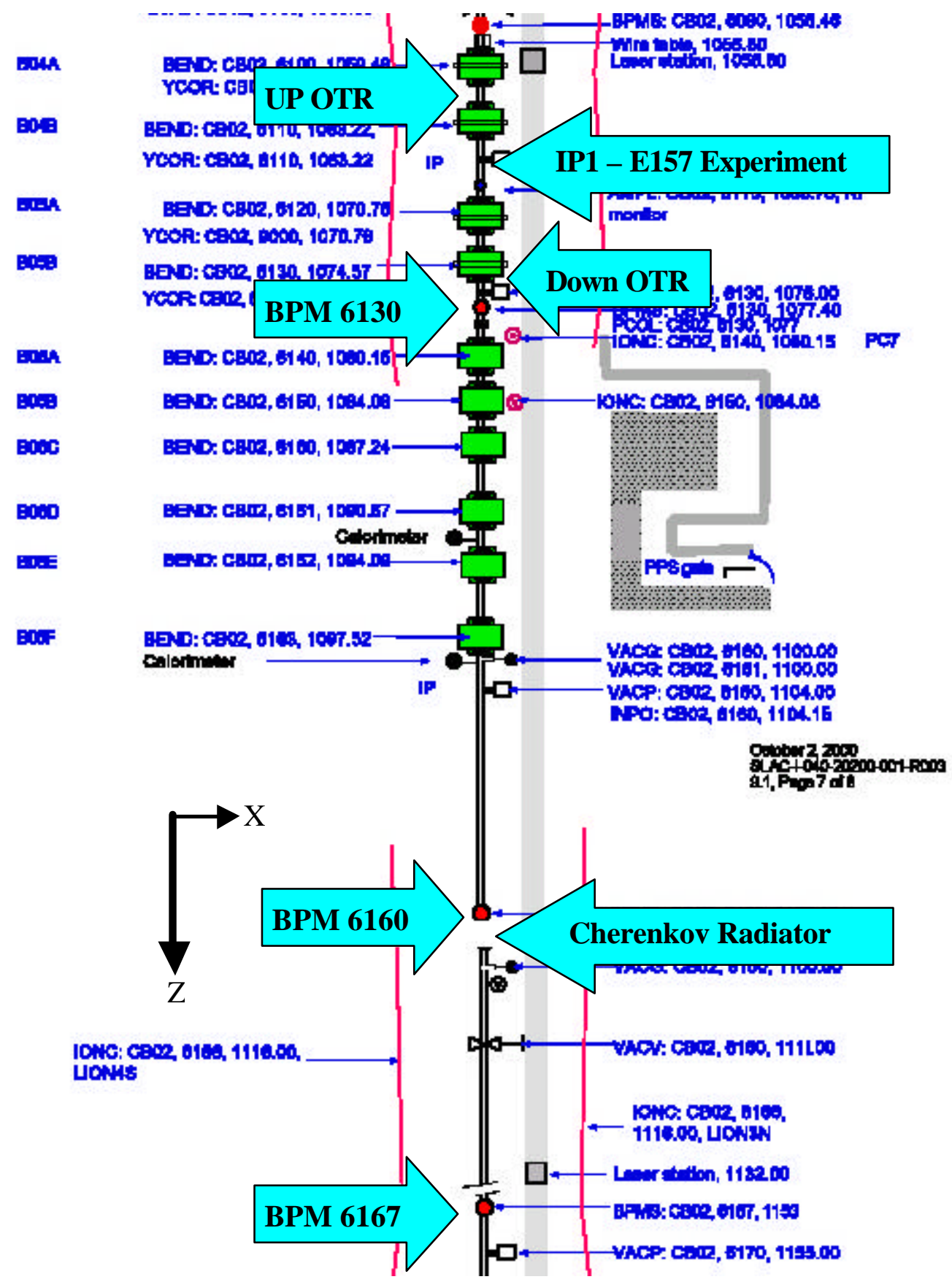

Figure 3.2.1 Diagram of the FFTB near IP1 with relevant diagnostic locations marked 


\section{The E157 Experiment}

\subsection{Lithium plasma source}

The two main components of the Li plasma source are the heat pipe oven and the ionizing laser. The heat pipe oven consists of a stainless steel tube wrapped in heater tapes. The inside is lined with a wire mesh and is partially filled with solid (at room temperature) Lithium. Water jackets are placed at each end of the oven. A helium buffer gas is used to constrain the $\mathrm{Li}$ vapor. The oven is heated to $\sim 750^{\circ} \mathrm{C}$ and a $\mathrm{Li}$ vapor is formed. The vapor flows from the center of the oven (where it is the hottest) to the water jackets (where it is the coolest). The mesh acts as a wick and transports the $\mathrm{Li}$ back towards the center of the oven. The Helium gas, in conjunction with the water jackets, constrains the $\mathrm{Li}$ vapor into a uniform column with sharp boundaries. The end product of this heat pipe is a $1.4 \mathrm{~m}$ long, uniform column of $\mathrm{Li}$ vapor at a density of $\sim 2 \times 10^{15} \mathrm{~cm}^{-3}$.

An argon-fluoride excimer laser provides an ultraviolet pulse (193nm, 6.45eV) to ionize the $\mathrm{Li}$ vapor via single photon absorption. The first ionization energy of $\mathrm{Li}$ is $5.392 \mathrm{eV}$ [25] with an ionization cross section of $1.8 \times 10^{-18} \mathrm{~cm}^{-2}$. A 10-20ns laser pulse is focused down the vapor column so that laser fluence is constant (photon absorption is counteracted by reduced spot size). At the entrance of the plasma the laser crosssection is approximately $16 \mathrm{~mm}^{2}$ whereas at the end it is approximately $8 \mathrm{~mm}^{2}$. Because the laser fluence determines the plasma density, simply changing the output pulse energy on the laser can vary the oven's plasma density. This results in a $1.4 \mathrm{~m}$ 
long plasma column of with a variable density up to $6 \times 10^{14} \mathrm{~cm}^{-3}$ as the laser energy is varied from 0-40 $\mathrm{mJ}$.

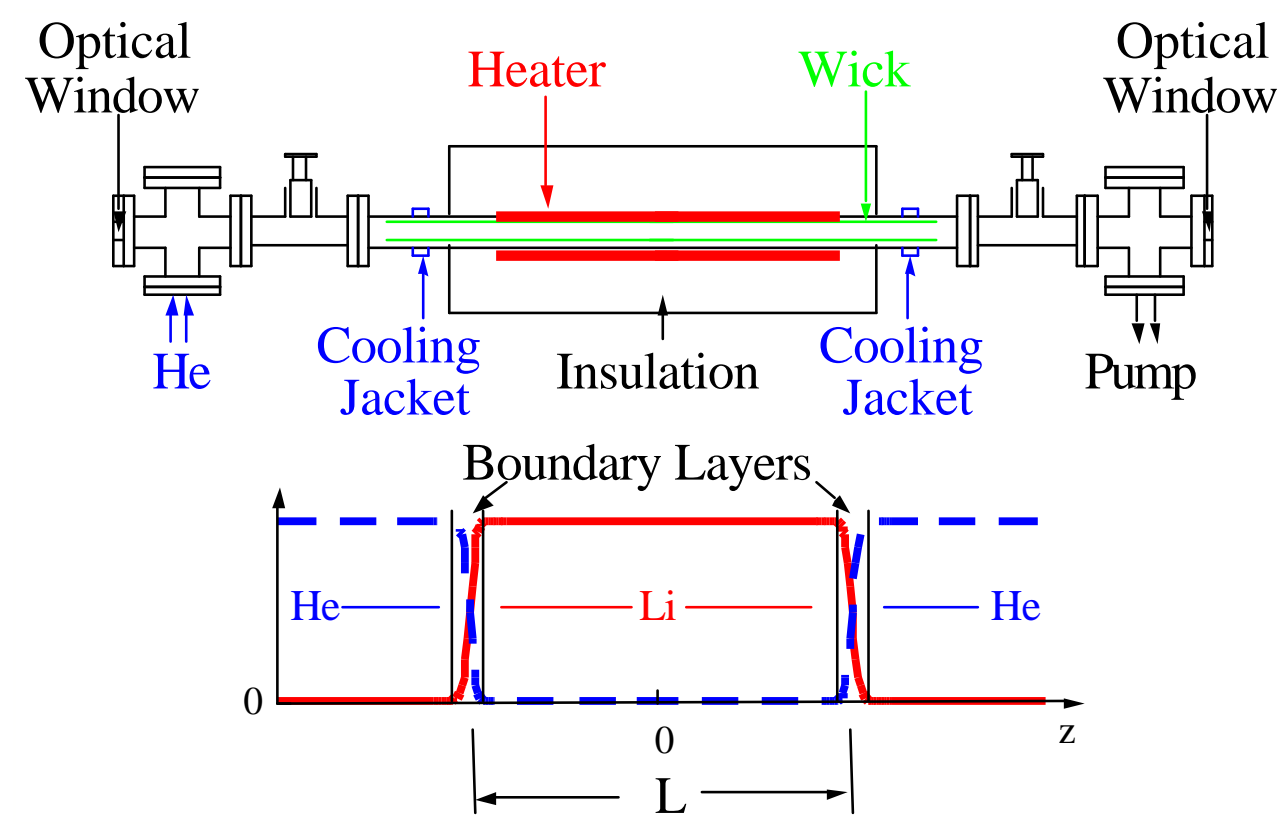

Figure 3.3.1 Diagram of Lithium Oven Setup and Depiction of Lithium Column

\subsection{Optical Transition Radiators (OTR)}

Two OTR diagnostics are employed in the E157 experiment, one $1 \mathrm{~m}$ before the plasma and one $1 \mathrm{~m}$ after the plasma. These diagnostics give us a single shot timeintegrated picture of the beam that allow us to measure the beam's spot size in both the $\mathrm{x}$ and $\mathrm{y}$ planes. The setup consists of a thin titanium foil placed at a $45^{\circ}$ angle in the beam line. An AF Micro-Nikkor $105 \mathrm{~mm}$ f/2.8D lens is used to image the OTR 
from the foil on to a 12-bit Photometrics Sensys CCD camera. The CCD has a pixel size of $9 \mu \mathrm{m} \times 9 \mu \mathrm{m}$ with an array size of $768 \times 512$. The spatial resolution of the setup is approximately $20 \mu \mathrm{m}$. A computer is used to read out the images and it can acquire date at $1 \mathrm{~Hz}$.

OTR is one mechanism by which a charged particle can emit radiation. The radiation is emitted when a charged particle passes from one medium into another. For our case, the electron beam is propagating in a vacuum and then it enters a titanium foil. When the beam is in vacuum it has certain field characteristics, and when it is inside the titanium foil it has different field characteristics. As the beam makes the transition into the foil, the fields must reorganize themselves. In the process of reorganization, some of the field is "shed" off. Optical transition radiation is this "shed" field [26].

OTR has been used extensively on low energy (MeV) beams, but people thought that it would not be a viable diagnostic for high energy $(\mathrm{GeV})$ beams. The OTR has a peak at angles ?=1/?, which is small for $30 \mathrm{GeV}$ beams $(?=60000)$. Because diffraction limited resolution goes as ?/?, the assumption was that high energy beams could not be resolved. The misconception was that ? is the numerical aperture of the lens, not the radiation source, and that there is significant radiation in the wings of the OTR distribution profile. The E157 collaboration has proved this to be correct by measuring spot sizes on the order of $30 \mu \mathrm{m}$. Figures 3.3.1 shows typical upstream and downstream OTR images. The graininess of the downstream OTR 
image is not from the beam, rather it results from the grain structure of the titanium foil. Figure 3.3.2 shows how the image analysis routines in Matlab extract spot size information from the images.

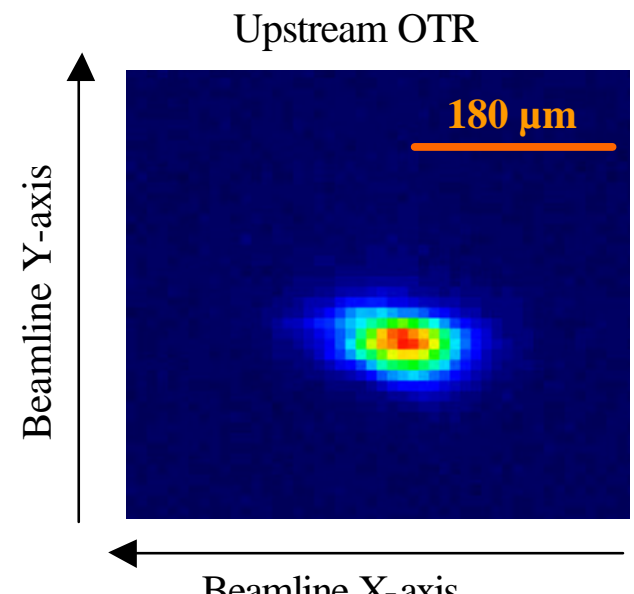

Beamline $\mathrm{X}$-axis

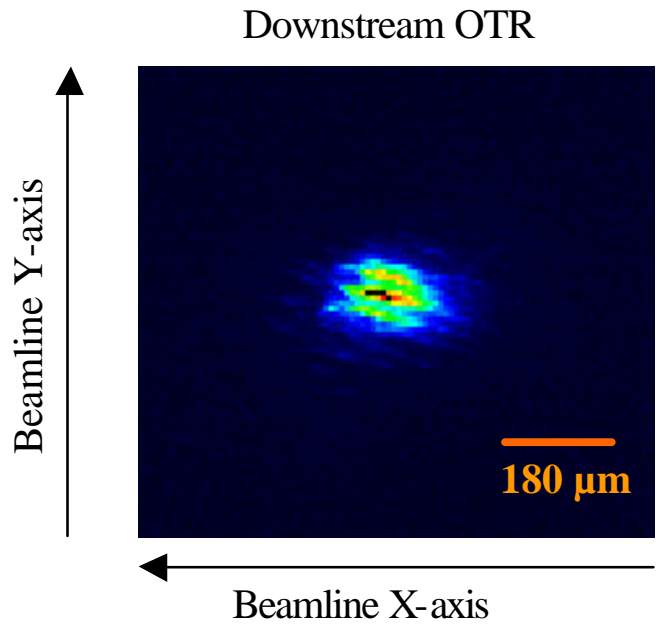

Figure 3.4.1 Upstream and Downstream OTR Images 


\section{Upstream OTR Image}

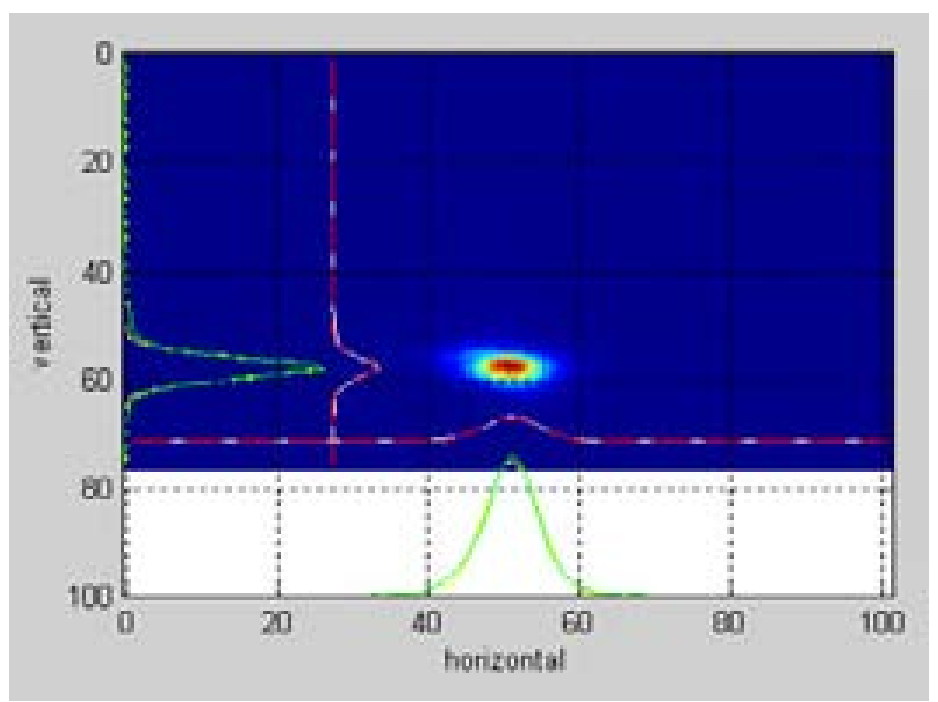

Downstream OTR Image

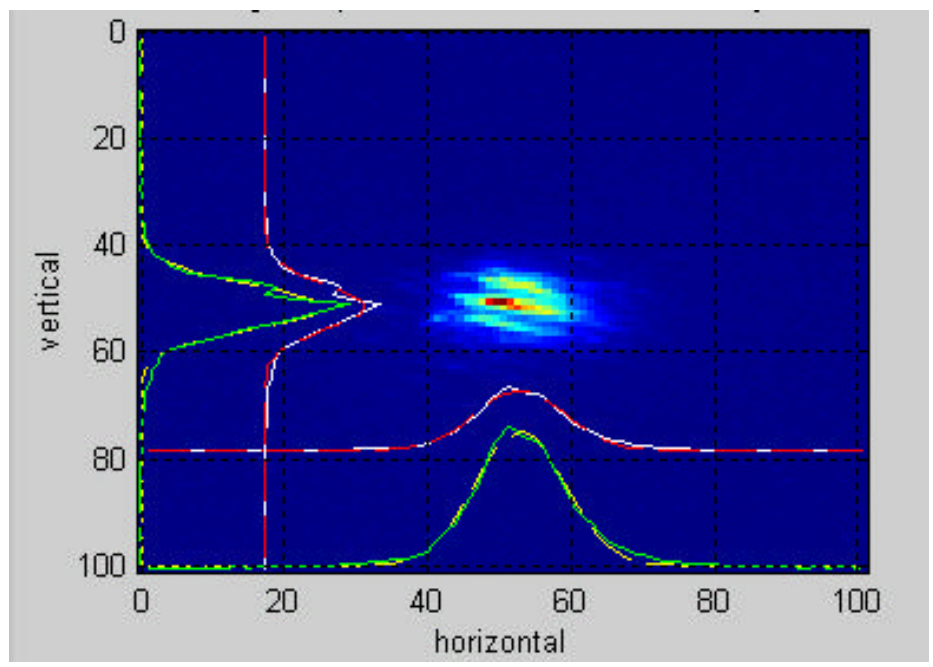

Figure 3.4.2 Image analysis of upstream and downstream OTR images. The white dashed line is the background level. The red line is a gaussian fit to the background level. The dashed yellow line is either a summation of the columns (for the vertical plane) or a summation of the rows (for the horizontal plane). The green lines are gaussian fits to the summation. The upstream image has a vertical spot size of 33.3 $\mu \mathrm{m}$ ( 3.7 pixels) and a horizontal spot size of $18 \mu \mathrm{m}$ ( 2 pixels). The downstream image has a vertical spot size of $37.8 \mu \mathrm{m}$ (4.2 pixels) and a horizontal spot size of $55.8 \mu \mathrm{m}$ (6.2 pixels). 


\subsection{Beam Position Monitors (BPMs)}

BPMs measure the beam's position by coupling to the beams electromagnetic field. They use four stripline antennas mounted in quadrature inside the beam pipe as shown in figure 3.5.1.

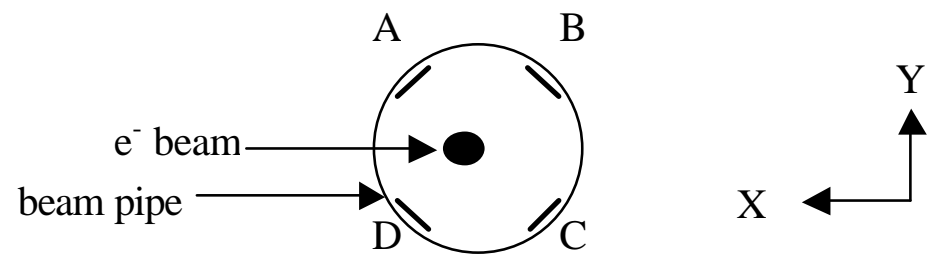

Figure 3.5.1. Typical Stripline BPM

The most common measurement is called the "difference over sum" method. In this method, the radio frequency signal is peak rectified and stretched. The output is then fed into circuitry that calculates positions from

$$
\begin{aligned}
& X=\frac{(A+D)-(B+C)}{A+B+C+D} \frac{1}{S_{x}} \\
& Y=\frac{(A+B)-(C+D)}{A+B+C+D} \frac{1}{S_{y}}
\end{aligned}
$$

where

$$
S_{x}(0,0)=S_{y}(0,0)=\left\{\begin{array}{l}
\frac{\sqrt{2}}{r} ; @ 45^{\circ} \\
\frac{1}{r} ; @ 0^{\circ}
\end{array}\right.
$$


In addition to calculating position, the BPMs can also be used to measure the beam current.

BPMs are positioned all along the beam line in the FFTB. Several are upstream of the plasma chamber and are used to align the beam with quadrupole magnets and our plasma chamber. Five BPMs located downstream of the plasma chamber are used to measure the trajectory of the beam as it exists the plasma.

\subsection{Aerogel Cherenkov Radiator}

Cherenkov radiation is emitted whenever charged particles pass through a medium with a velocity that exceeds the velocity of light in that medium.

$$
\mathrm{v}>\mathrm{v}_{\mathrm{t}}=\mathrm{c} / \mathrm{n}
$$

where $\mathrm{v}$ is the particle's velocity (in our case $\mathrm{v}=\mathrm{c}$ ), $\mathrm{v}$ is the threshold velocity, and $\mathrm{n}$ is the index of refraction of the medium. One can see that Cherenkov radiation will be emitted anytime a relativistic beam passes through any medium $(n>1)$. The light is emitted at a constant angle with respect to the particle's trajectory.

$$
\cos (\delta)=\frac{v_{t}}{v}=\frac{c}{v n}=\frac{1}{n \beta}=\frac{1}{n}(\text { for } v=c)
$$

This emission is similar to the bow shock created during supersonic flight.

In our experiment, we use the Cherenkov light as part of our energy gain diagnostic. The electron beam exists the plasma and drifts through an energy dispersive magnet. The beam then passes through the aerogel and emits Cherenkov 
radiation. The light is transported approximately $15 \mathrm{~m}$ to an optical table outside of the FFTB. The light passes through a beam splitter and a small fraction of the light is sent to a $\mathrm{CCD}$ camera for time-integrated images of the beam figure 3.6.1 (similar to the OTR images). The remaining light passes through a beam splitter again and one arm is rotated 90 degrees. The two paths are recombined and sent to the streak camera. The 90 degree rotation allows us to streak both the energy dispersive plane (y-axis) and the non-dispersive plane (x-axis) simultaneously figure 3.6.2. Figure 3.6.3 shows the layout of the optical table. The streak camera has a temporal resolution of one picosecond and a spatial resolution of $\sim 100 \mu \mathrm{m}$. My analysis will use the nondispersive plane on the streak camera in order to resolve head-tail offsets.

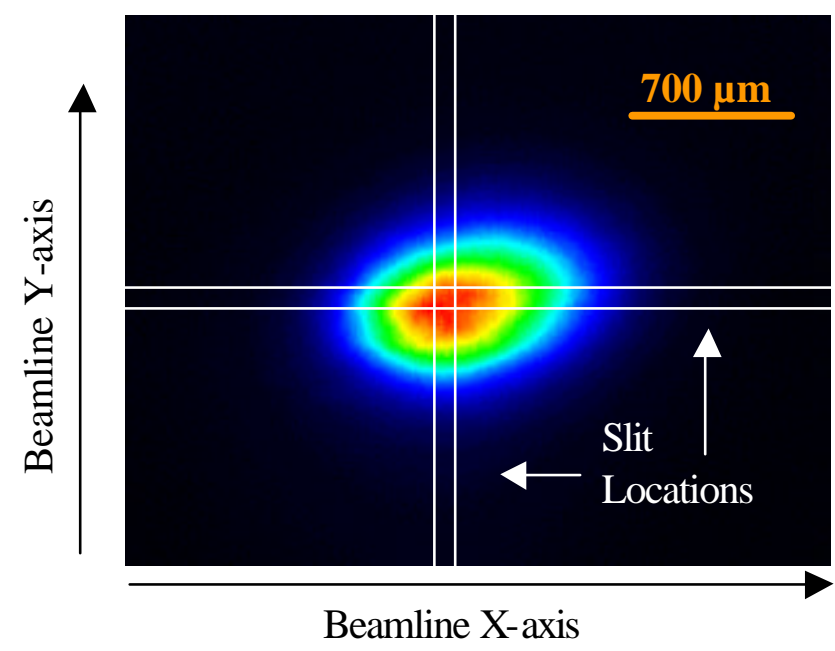

Figure 3.6.1 Time Integrated Cherenkov Image depicting the parts of the beam seen by the horizontal and vertical slits on the streak camera 


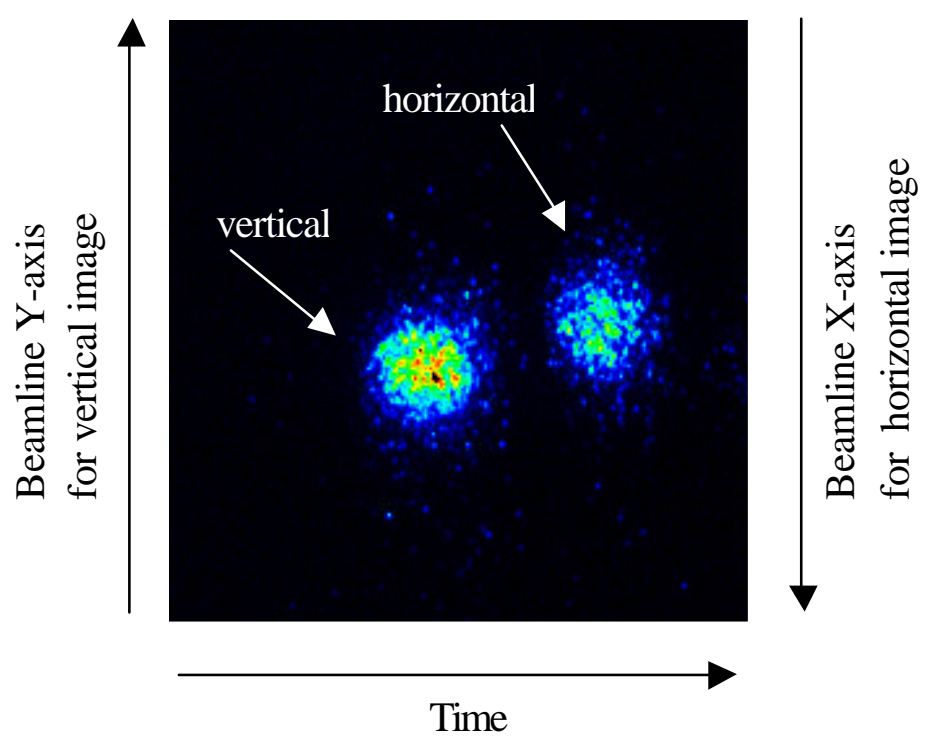

Figure 3.6.2 Typical Streak Camera image showing both the horizontal and vertical streaks 

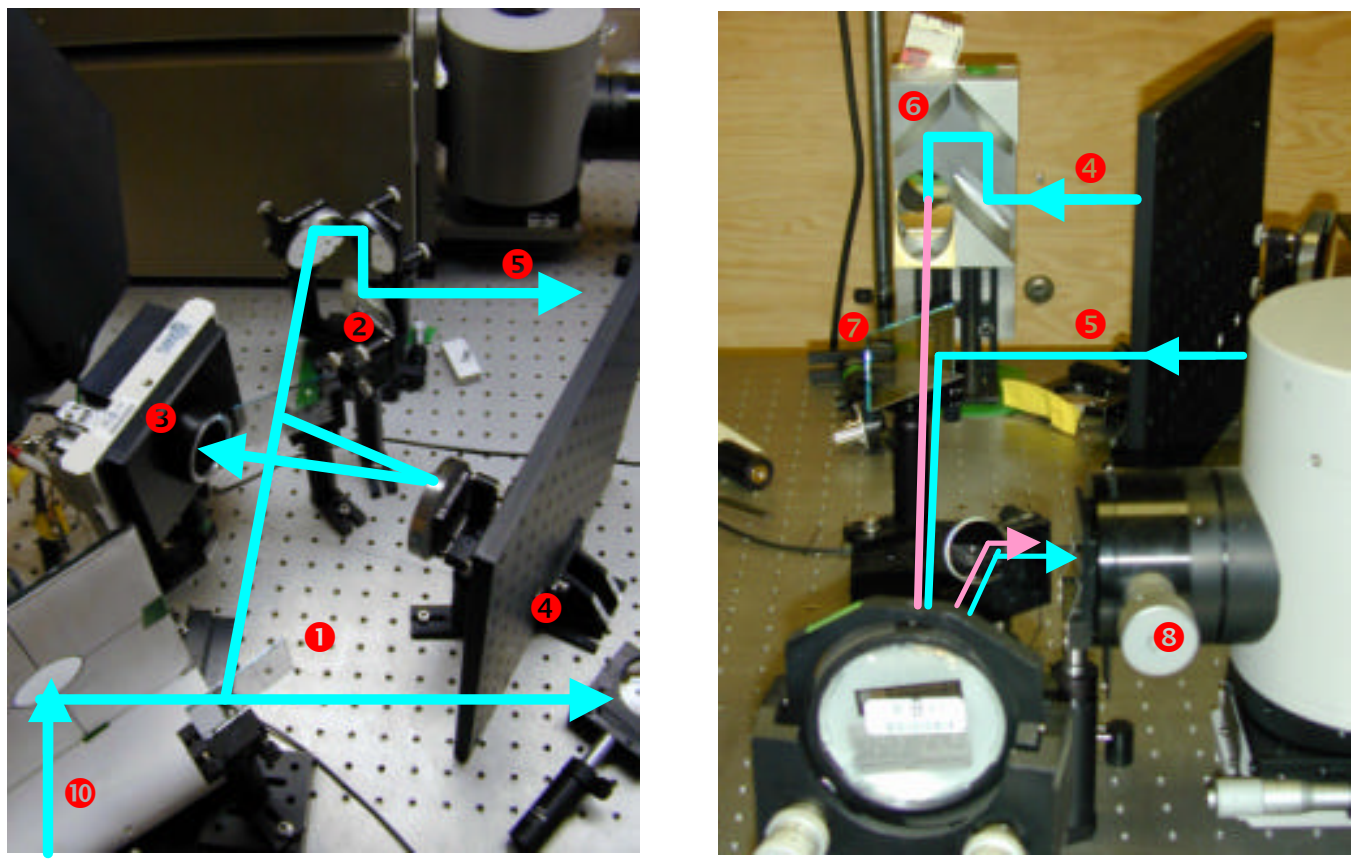

Figure 3.6.3 Diagram of Cherenkov radiation detector setup. The light radiated from the aerogel enters the setup at $\mathbb{1 0}$. It is reflected off of a mirror towards beam splitter (1. Part of the light is sent to the image rotator 6 via path 4 while the other part is directed towards beam splitter 2. Part of light off of beam splitter 2 is sent to a CCD camera 3 for time integrated Cherenkov images while the rest is directed towards beam splitter 8 via path $\boldsymbol{5}$. The image on path 4 passes through an image rotator $\boldsymbol{6}$ where it is rotated 90 degrees (the unrotated path is shown in light blue while the rotated image path is shown in purple). Both the unrotated and rotated images are combined at beam splitter $\boldsymbol{\nabla}$. The combined images (slightly delayed in time from each other) are then sent to the streak camera 8 for time-resolved analysis. 


\section{Results}

\subsection{Initial Beam Condition}

One of the major highlights of the E157 experiment was the advance made in beam diagnostics. These diagnostics allowed the accelerator physicists to see details of the beam dynamics they had not seen before. The lowest order concern for the experiment was delivering a beam to our plasma with a specified energy and spot size. These requirements were readily attainable since the energy was determined by the linac settings and the spot size was determined by adjusting the beam optics to focus the beam at the plasma entrance. With the knowledge that the electron hosing instability might be a problem for our experiment, we were also concerned about a longitudinal transverse correlation on the beam.

With the available E157 diagnostics, a beam tilt could be quantified by time resolving laser off (i.e. no plasma) data. Because the streak camera is located after the energy (or vertical plane) dispersive bend magnet, we will look at streak data from the horizontal plane (no dispersion). The first step is to slice up the streaked image in 1ps bins. Next, analyze each slice to find its mean position. Finally, a tilt implies that the mean position of each slice will change in time. Figure 4.1.1 shows a streak camera image, where the horizontal streak image has been selected. The lines on the image illustrate how the data analysis software slices up the time-resolved image. 
Horizontal Laser Off Streak Data

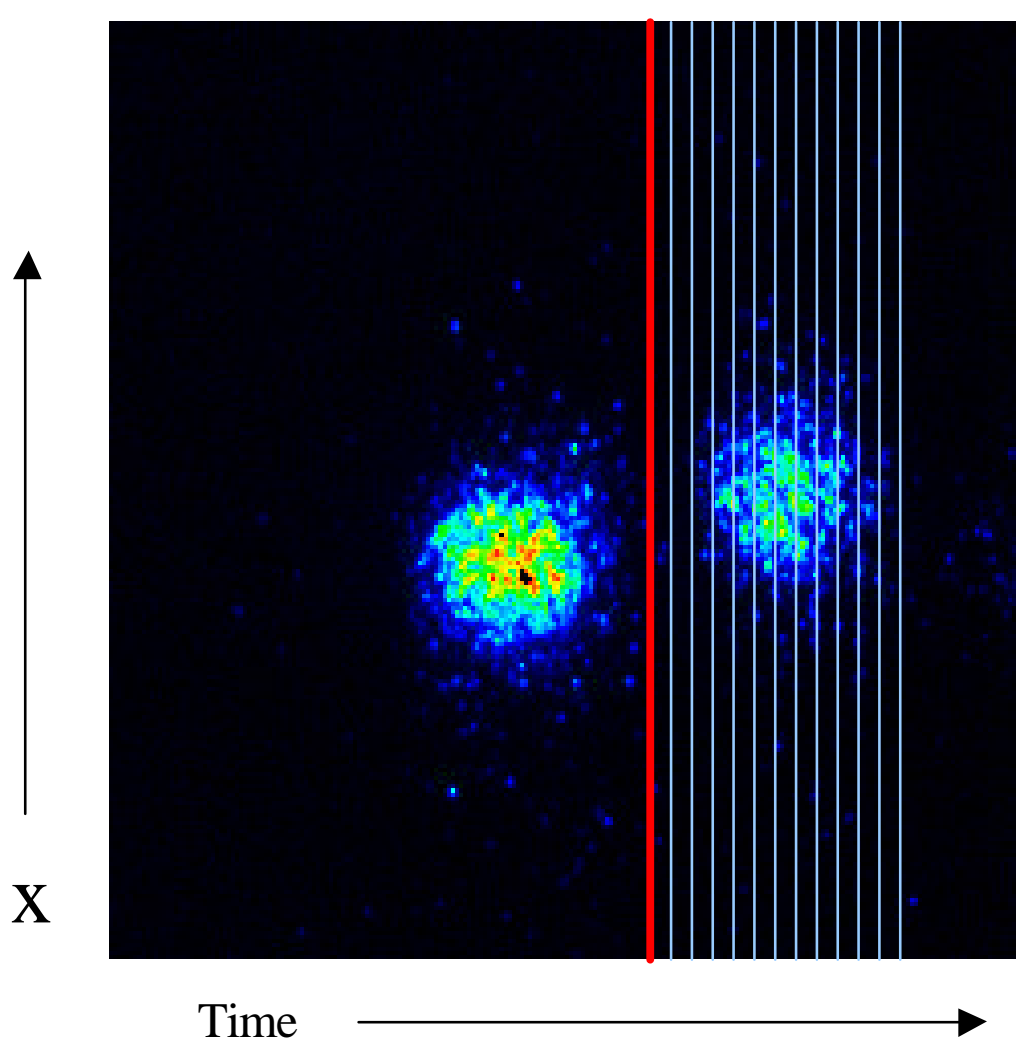

Cut beam up into slices

Analyze each slice to find $\langle x\rangle$ $>$ A tilt implies $\langle x\rangle$ will move in time

Figure 4.1.1 Streak Camera Data

The results of the analysis for two single events are plotted with the xaxis being time and the yaxis being mean position. $-4 \mathrm{ps}$ is the head of the beam, and $+5 \mathrm{ps}$ is the tail end of the beam. To help visualize the beam tilt, imagine that you are flying down the 
beam line looking down at the electron bunch. You will see the back of the bunch is not in line with the head of the bunch, rather it is displaced to the side.

\section{Analysis of Single Shots}

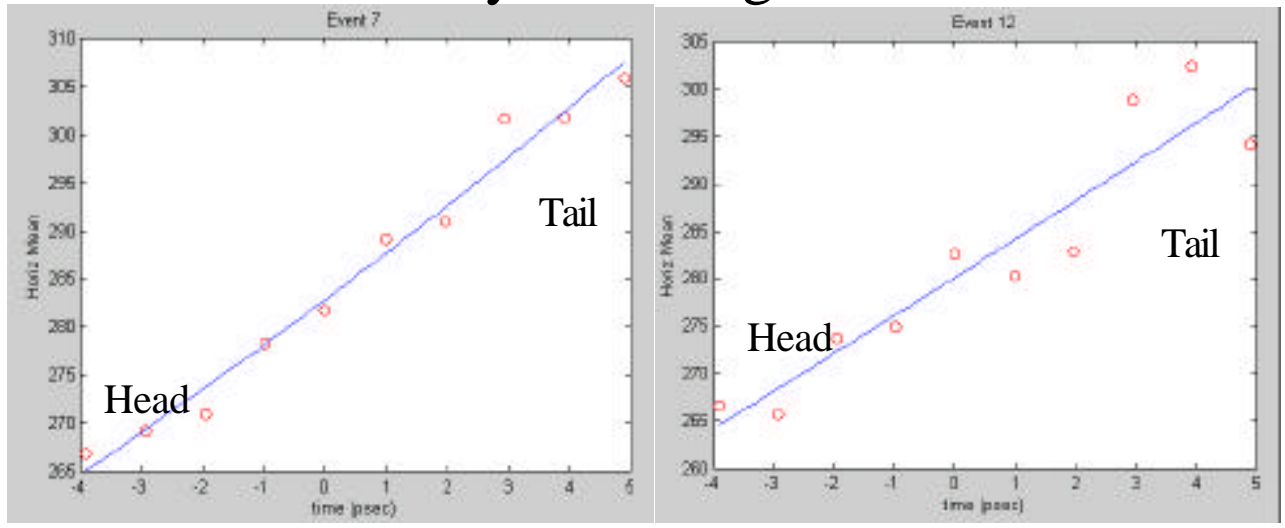

Figure 4.1.2 Tilted Beams $\left(\mathrm{N}=2 \times 10^{10} \mathrm{e}^{-} ; \mathrm{s}_{\mathrm{xDN}}=110 \mu \mathrm{m}\right)$

The total results for a single run of 25 consecutive shots are given in figure 4.1.3. Every data point is plotted for each picosecond bin. The solid line drawn through the points represent the mean of all 25 shots for each time step. The two dashed lines represent the mean \pm rms for each time step.

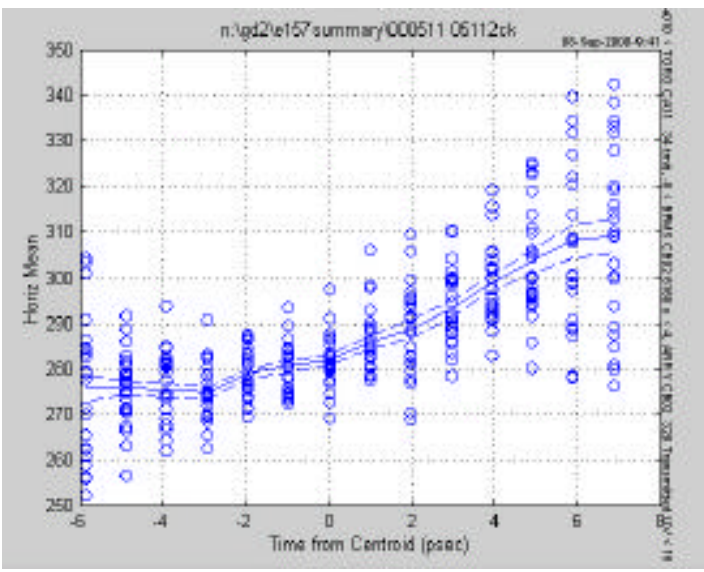

Figure 4.1.3 Average of 25 laser off shots $\left(\mathrm{N}=2 \times 10^{10} \mathrm{e}^{-} ; \mathrm{s}_{\mathrm{xDN}}=110 \mu \mathrm{m}\right)$ 
The bunch length corresponds to a $s_{z}$ of about 2 ps. This gives a beam tilt of $84 \mu \mathrm{m} / \mathrm{s}$ or $197 \mu \mathrm{m}$ at FWHM. The beam spot size, measured from the time integrated Cherenkov image, was $\mathrm{s}_{\mathrm{x}}=560 \mu \mathrm{m}$ or $\mathrm{FWHM}=1316 \mu \mathrm{m}$. The conclusion we draw from this is that the beam is tilted.

The measured tilt corresponds to the beam parameters at the aerogel that is 12 meters downstream from the plasma chamber. What we are interested in is the beam tilt at the entrance of the plasma. Ideally we would move the aerogel and the streak camera to the plasma entrance. This was not possible, so we approximate the beam tilt at the plasma entrance. We do this by fitting the beam envelope equation

$$
\begin{aligned}
& \frac{\partial^{2} \sigma(z)}{\partial z^{2}}+\left[K^{2}-\frac{\varepsilon_{N}^{2}}{\gamma^{2} \sigma^{4}(z)}\right] \sigma(z)=0 \\
& K= \begin{cases}0 & \text { no plasma } \\
\frac{\sigma_{p}}{c \sqrt{2 \gamma}} & \text { in a plasma }\end{cases} \\
& \varepsilon_{N} \quad \text { Normalized emittance }
\end{aligned}
$$

to the three known spot sizes (upstream OTR, downstream OTR, and time-integrated Cherenkov) in order to find the spot size at the plasma entrance. From the fitting procedure, the minimum spot size turns out to be $32 \mu \mathrm{m}$. The location of the beam waist is approximately $20 \mathrm{~cm}$ from the plasma entrance. This is shown in figure 4.1.4. 


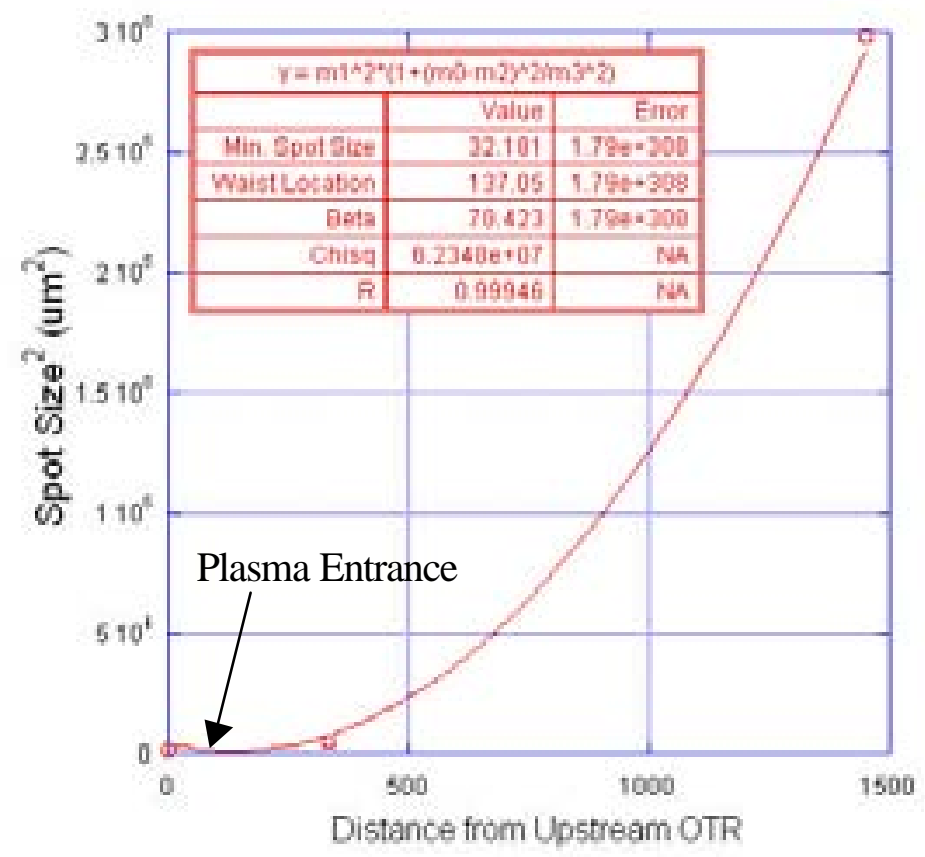

Figure 4.1.4 Fit to envelope equation

After determining the spot size at the plasma entrance, we determine the tilt at the plasma entrance, $R_{0}$, by scaling the tilt at the aerogel, $R_{1}$, by the ratio of the two beam spot sizes.

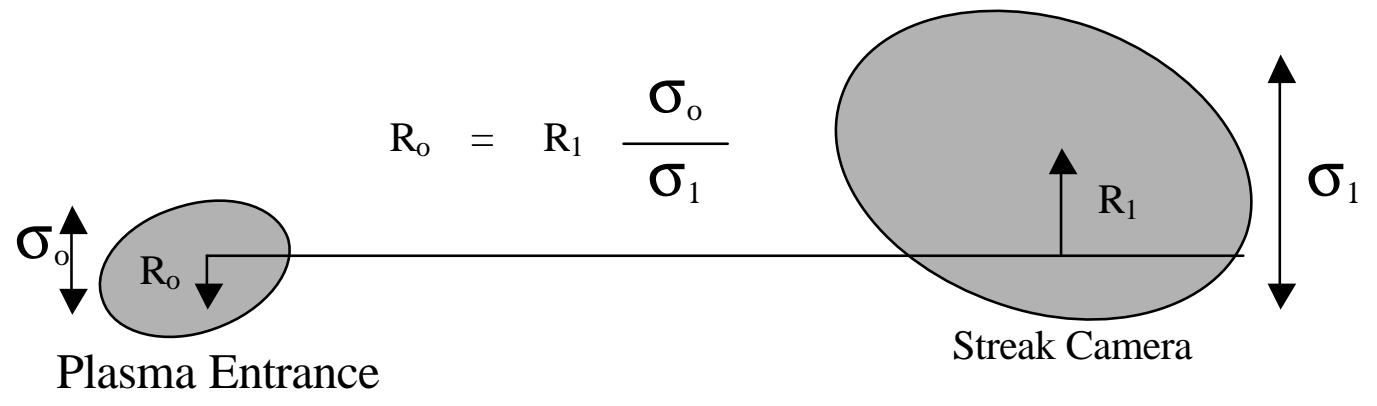

Figure 4.1.5 Scaling of Beam Tilt 
The values for the offset at the plasma entrance for the 25 shots are

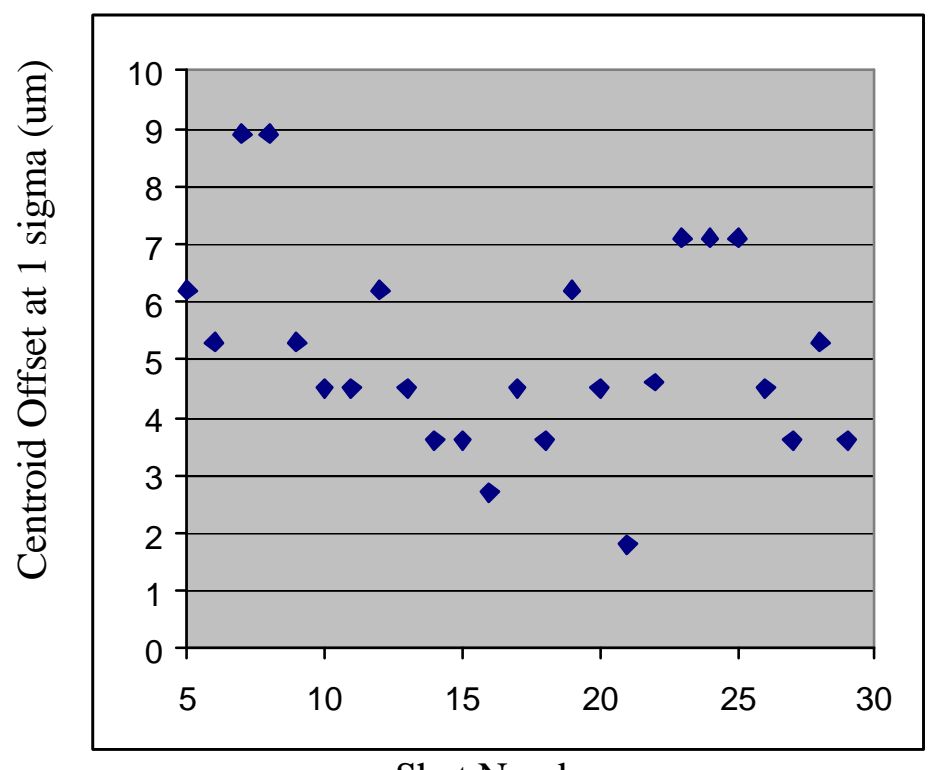

Shot Number

Figure 4.1.6 Centroid offset at plasma entrance $\left(\mathrm{N}=2 \times 10^{10} \mathrm{e}^{-} ; \mathrm{s}_{\mathrm{xUP}}=36 \mu \mathrm{m}\right)$

\subsection{Centroid Oscillations}

Although our analysis is limited to the horizontal plane (due to the dispersive bending magnet), a beam tail generally lies in both the vertical and horizontal planes. Figure 4.2.1 shows two upstream OTR images of beams with large tails. With the knowledge that the beam coming into the plasma is tilted, we would expect the trajectory of the exiting beam centroid to oscillate (equation 2.1.17). The data in figures 4.2.2 and 4.2.3 was energy scan data. The plasma density (x-axis) was varied by varying the ionizing laser energy. Figure 4.2.2 shows centroid oscillations in both the horizontal and vertical planes just after the plasma on BPM 6130. Figure 4.2.3 
shows the horizontal deviation of the beam centroid from the laser off position on the downstream OTR (DNOTR). In general, neither the spot size nor the centroid oscillations in both the horizontal (x) nor the vertical (y) planes were in exact phase with respect to each other because of the large difference in the beam emittance in these two planes.

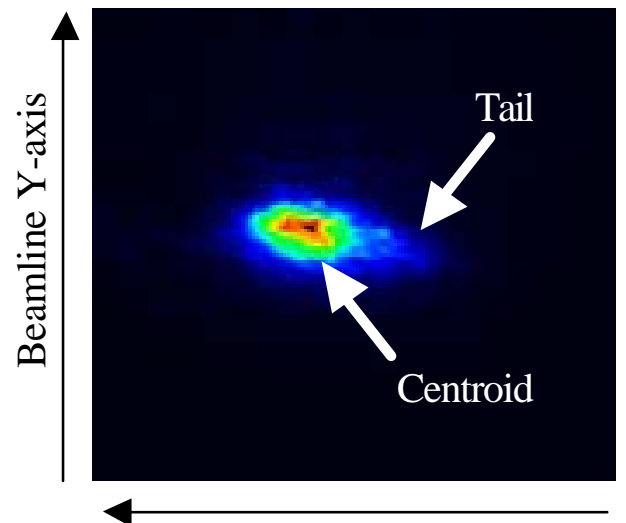

Beamline $\mathrm{x}$-axis

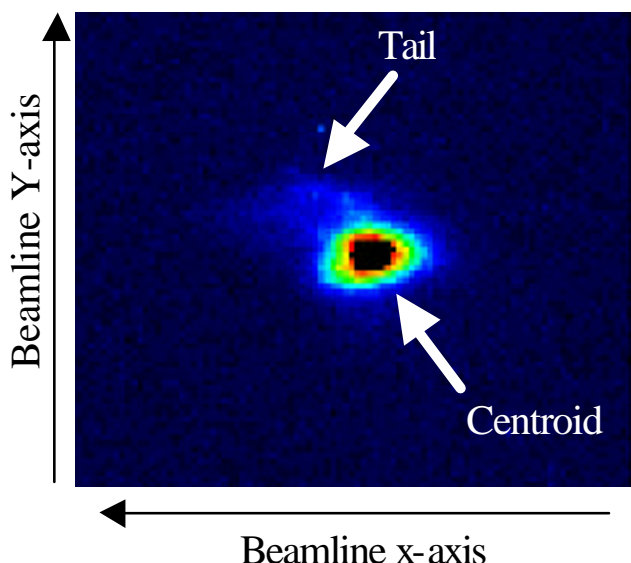

Beamline $\mathrm{x}$-axis

Figure 4.2.1 Upstream OTR images showing a tail that lies in both the horizontal and vertical plane (with respect to the beam centroid).
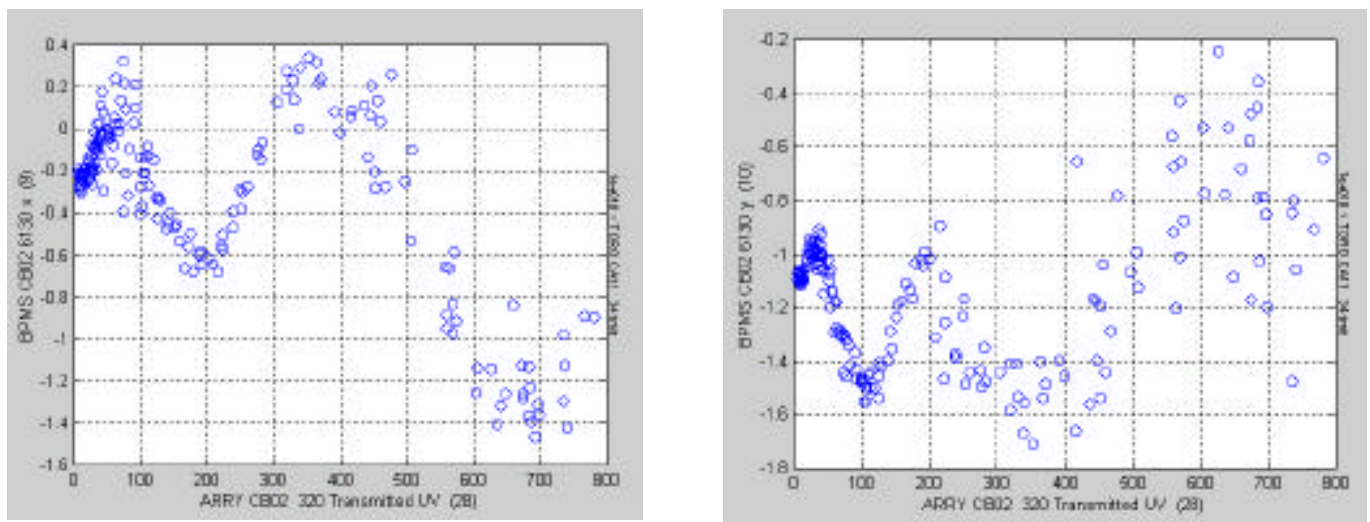

Figure 4.2.2 Centroid Oscillations on BPM 6130 for both the horizontal and vertical planes. The $\mathrm{x}$-axis is the laser energy (plasma density) and the $\mathrm{y}$ axis is the position of the beam centroid measured by the BPM. 


\section{Centroid Displacement at DNOTR}

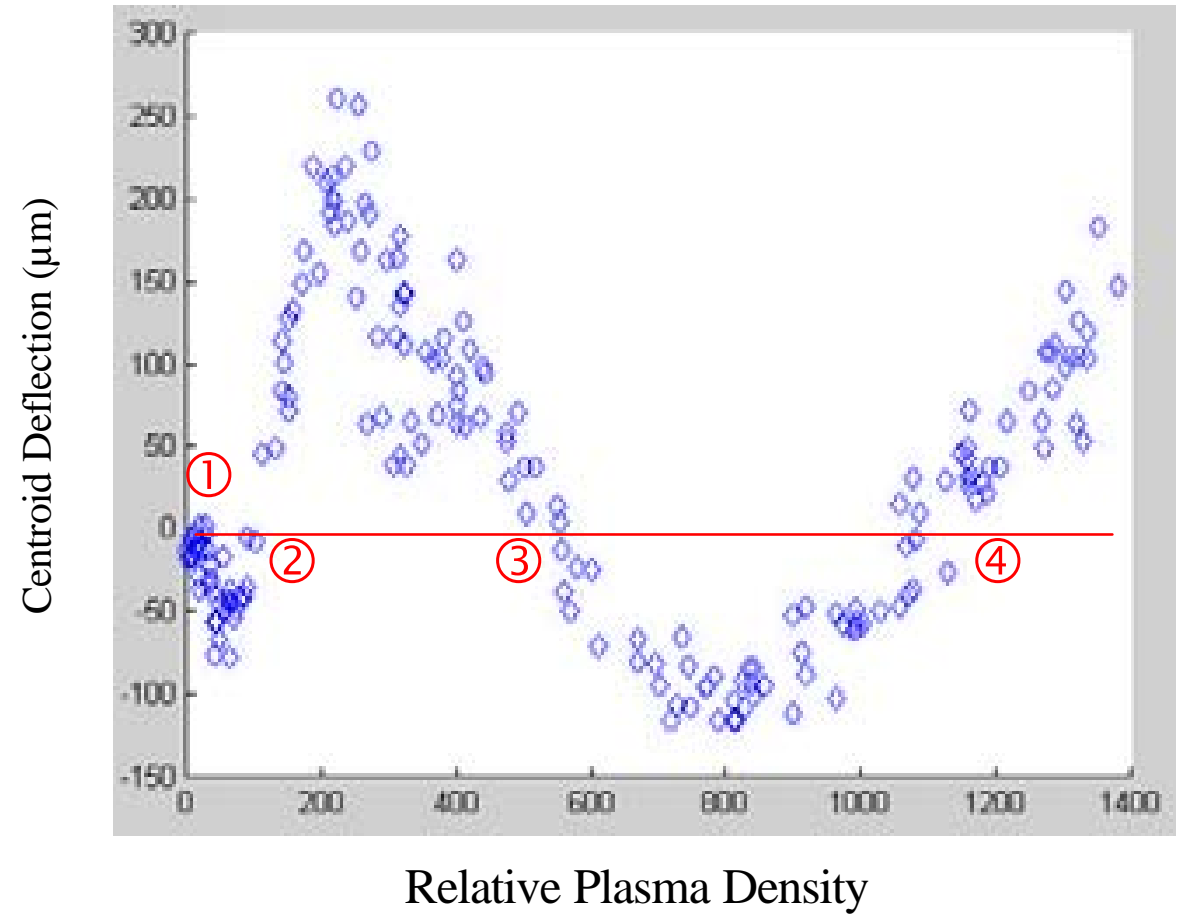

Figure 4.2.3 Centroid Oscillations $\left(\mathrm{N}=2.1 \times 10^{10} \mathrm{e}^{-} ; \mathrm{s}_{\mathrm{xUP}}=55 \mu \mathrm{m}\right)$

The centroid starts out in the same position as laser off, and then it goes through oscillations as the plasma density is increased. The four locations numbered 1-4 correspond to the zero crossings of the centroid oscillation.

In addition to the centroid oscillations, the beam experiences a transverse focusing force called betatron oscillations [27]. The plasma column acts as a thick lens whose focusing force is a function of plasma density. In the energy scan data, the beam underwent multiple betatron oscillations as can be seen from figure 4.2.4. 


\section{Spot Size at DNOTR vs. Plasma Density}

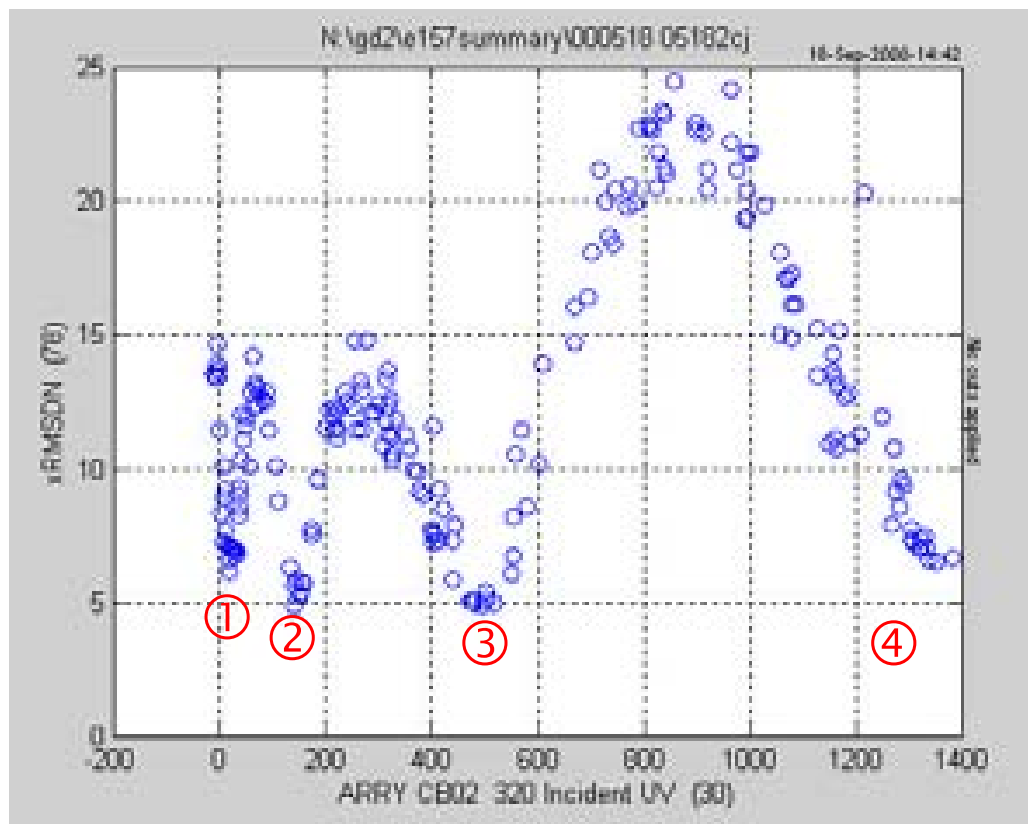

Figure 4.2.4 Betatron oscillations $\left(\mathrm{N}=2.1 \times 10^{10} \mathrm{e}^{-} ; \mathrm{s}_{\mathrm{xUP}}=55 \mu \mathrm{m}\right)$

The $\mathrm{x}$-axis is plasma density and the $\mathrm{y}$-axis is the beam's spot size $1 \mathrm{~m}$ after the plasma. As the plasma density is increased, the beam goes through multiple focii that are numbered 1-4. You can see that the pinch (or focus) densities shown in figure 4.2.4 correspond to the zero crossings of the centroid oscillations shown in figure 4.2.3. This is as expected since the betatron oscillations are at twice the frequency of the offset centroid oscillations. The cartoon in figure 4.2 .5 will describe why these two phenomena are related. 


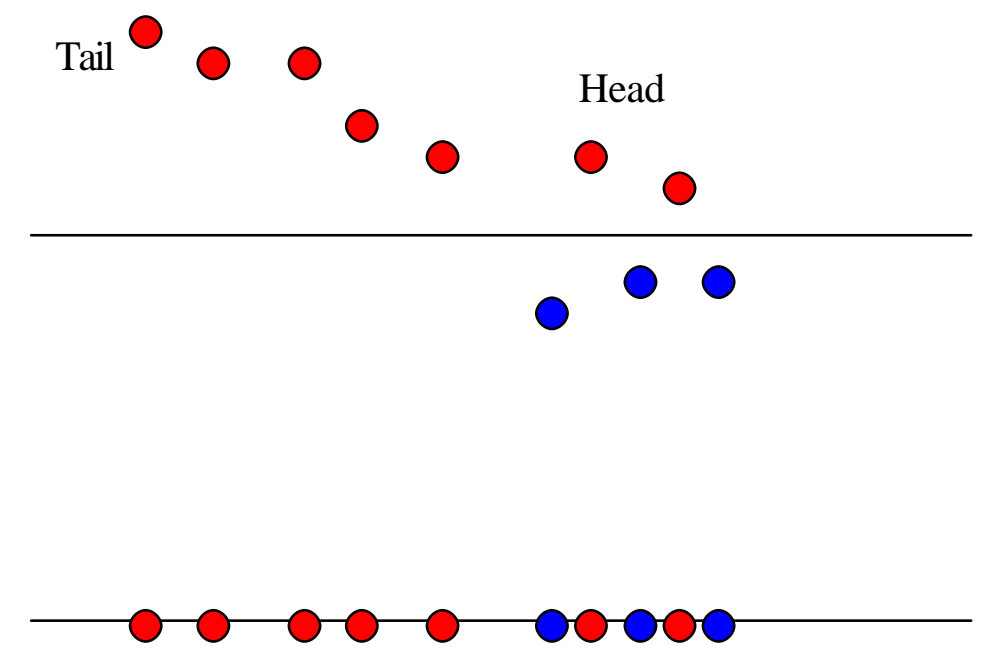

1) Initial Beam Condition at the plasma exit. Note red electrons start off above the axis and blue ones below

2) At the first pinch - all electrons moved towards the axis. Notice the zero crossing of the centroid oscillation. The electrons have a maximum transverse or perpendicular momentum. This beam will defocus at the Cherenkov detector

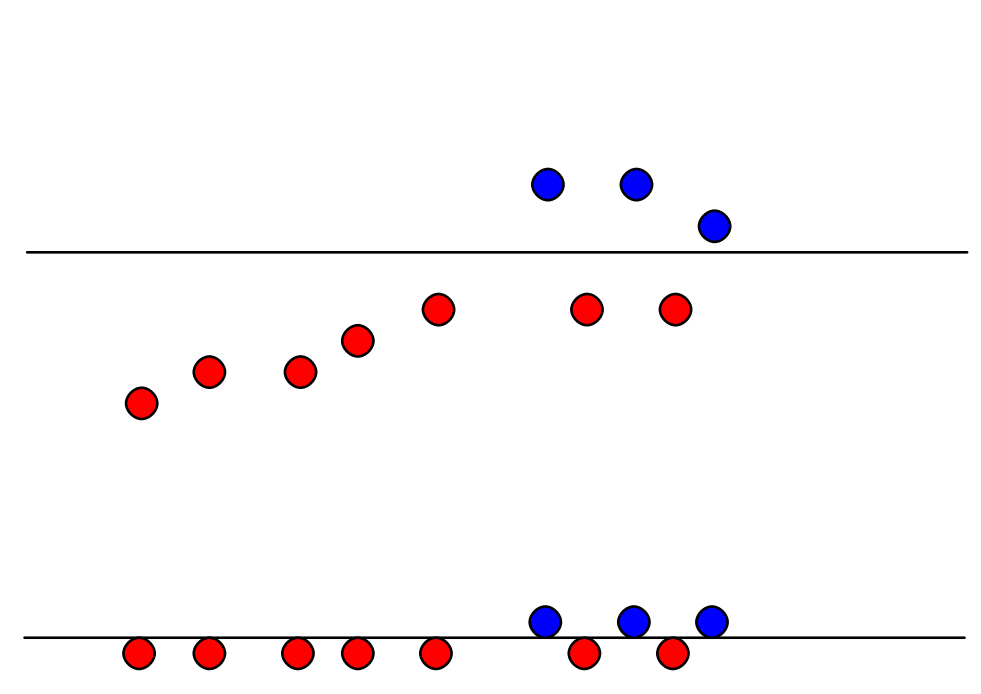

3) Moving away from the pinch. The beam has blown back up to its original size (one oscillation) and the centroid (tail) has made half an oscillation. This is the "so called" $p$ phase advance condition. The tail is now below the axis and the electrons have a minimum transverse or perpendicular momentum.

4) The beam goes through a second pinch and the centroid goes through a second zero crossing. Again the electrons have a large perpendicular momentum at the plasma exit.

Figure 4.2.5 Single electron motion within the beam at the plasma exit

As one can see, all electrons are oscillating across the axis. The difference is that when there are electrons on both sides of the axis, the beam appears to be focusing and defocusing whereas the tail electrons only appear to oscillate across the axis. 


\subsection{Tail Flipping}

Following the above discussion, the dynamics of the beam can be studied by temporally resolving the beam near a pinch at the Cherenkov detector. This corresponds to a multiple of $\mathrm{p}$ phase advance for the beam envelope as it goes through the plasma since a well focused beam at the Cherenkov is only obtained when the beam exits the plasma more-or-less as it entered. As the beam passes through a pinch, the tail should flip from one side of the head to the other. Data was analyzed from both the $4^{\text {th }}$ pinch and the $3^{\text {rd }}$ pinch. The data was divided into bins according to proximity to the pinch. In each bin, the shots were analyzed to determine the horizontal mean position vs. position in the beam (head, tail, centroid, etc.) The data

shows the tail moving from one side of the head to the other at the $3^{\text {rd }}$ pinch. At the $4^{\text {th }}$ pinch, the tail goes back to its original side.

Figures 4.3.1 and 4.3.2 show how the data was binned for analysis. The bottom red line corresponding to xRMS of 36 denotes the laser off spot size. Shots with spot sizes below this line are considered to be at the transparency condition. The upper horizontal red line is an arbitrarily chosen criteria to say that any shots with a spot size above this line are defocusing. The vertical lines separate defocused shot into group that are either coming into or exiting the pinch. Figure 4.3.3 gives justification for the $3 \mathrm{rd}$ and $4^{\text {th }}$ pinch locations. Figures 4.3 .5 and 4.3.6 show the tail flipping at the different pinches. 


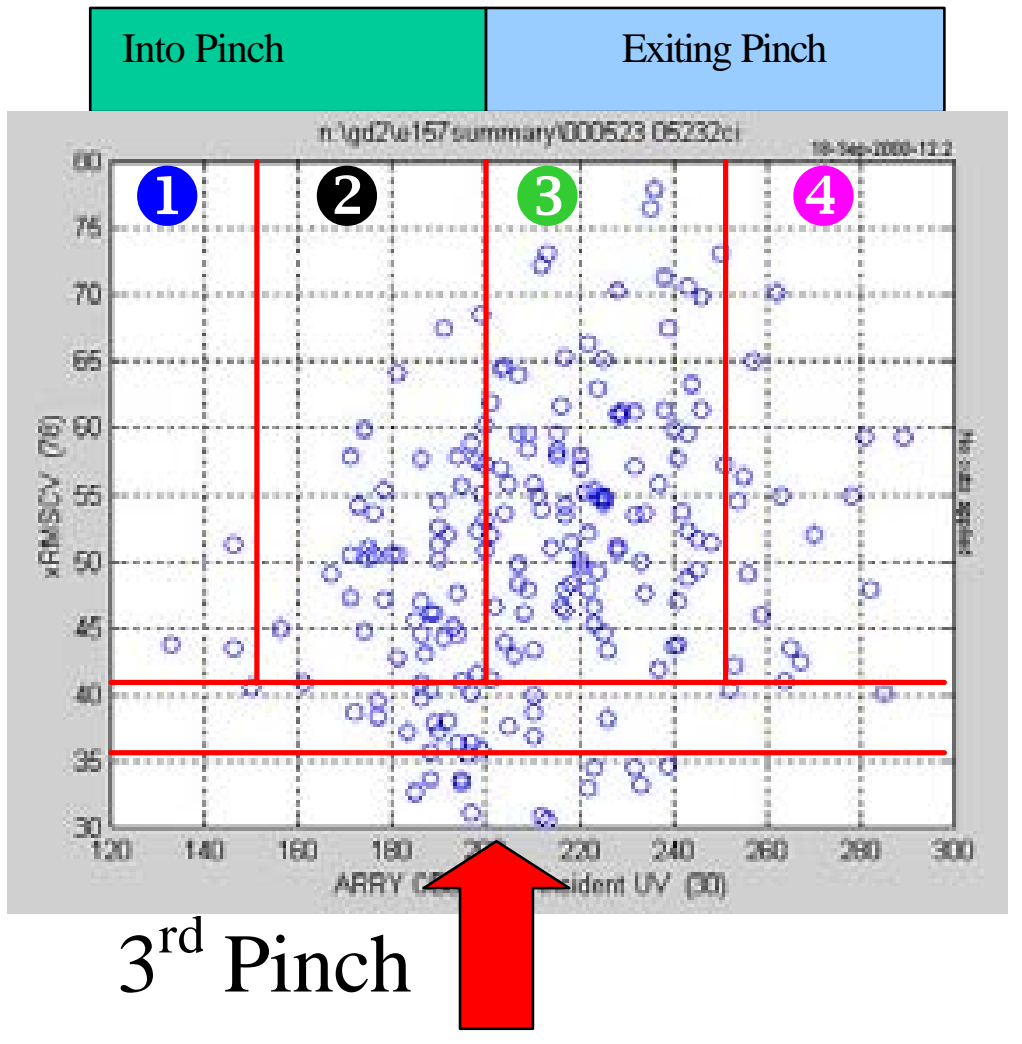

Figure 4.3.1 Spot size data from the $3^{\text {rd }}$ pinch showing the bins numbered 1-4. These bins are used in figure 4.3.5 $\left(\mathrm{N}=1.95 \times 10^{10} \mathrm{e}^{-} ; \mathrm{s}_{\mathrm{xUP}}=56 \mu \mathrm{m}\right)$ Nominal plasma density for the $3^{\text {rd }}$ pinch is about $1 \times 10^{14} \mathrm{~cm}^{-3}$. 


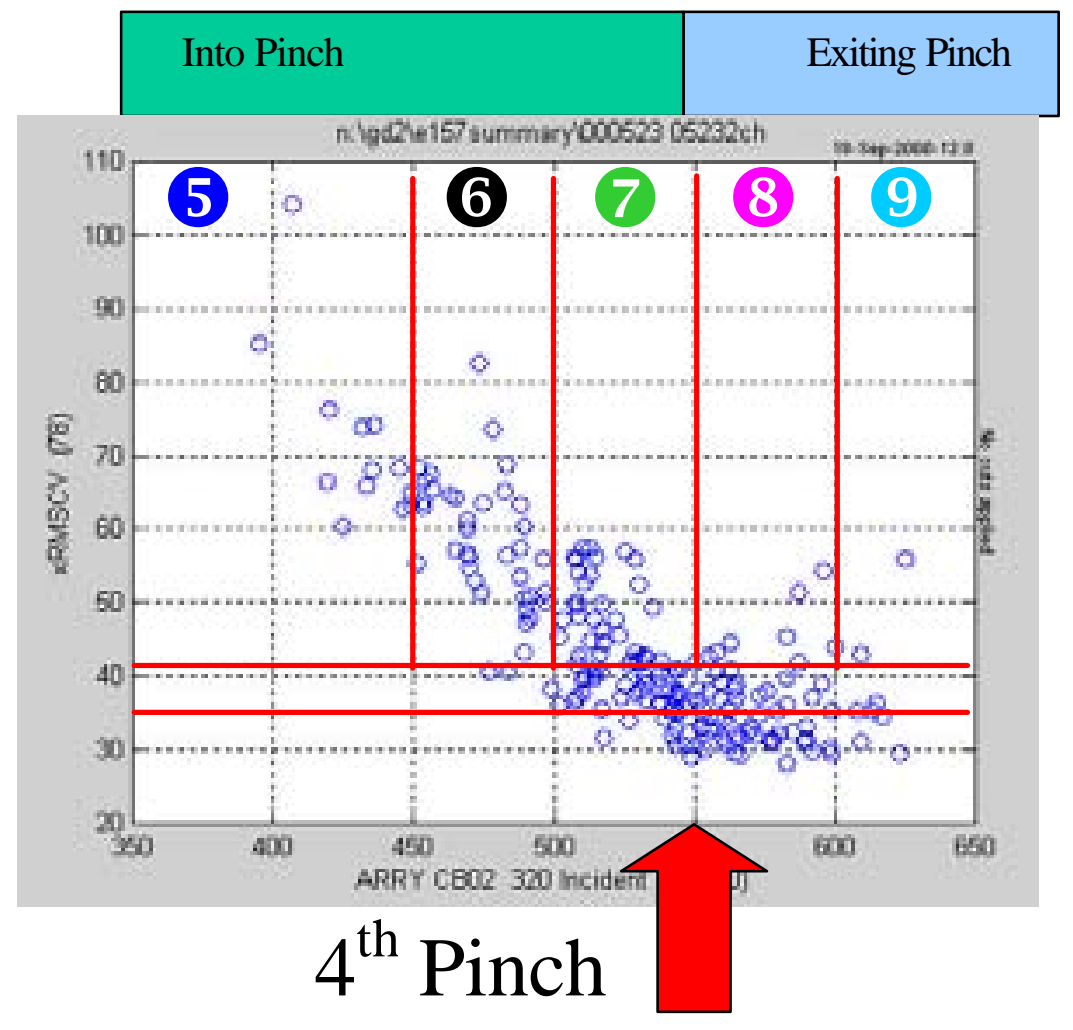

Figure 4.3.2 Spot size data from the $4^{\text {th }}$ pinch showing the bins numbered 5-9. These bins are used in figure 4.3.6. $\left(\mathrm{N}=1.95 \times 10^{10} \mathrm{e}^{-} ; \mathrm{s}_{\mathrm{xUP}}=54 \mu \mathrm{m}\right)$ Nominal plasma density for the $4^{\text {th }}$ pinch is about $2 \times 10^{14} \mathrm{~cm}^{-3}$. 

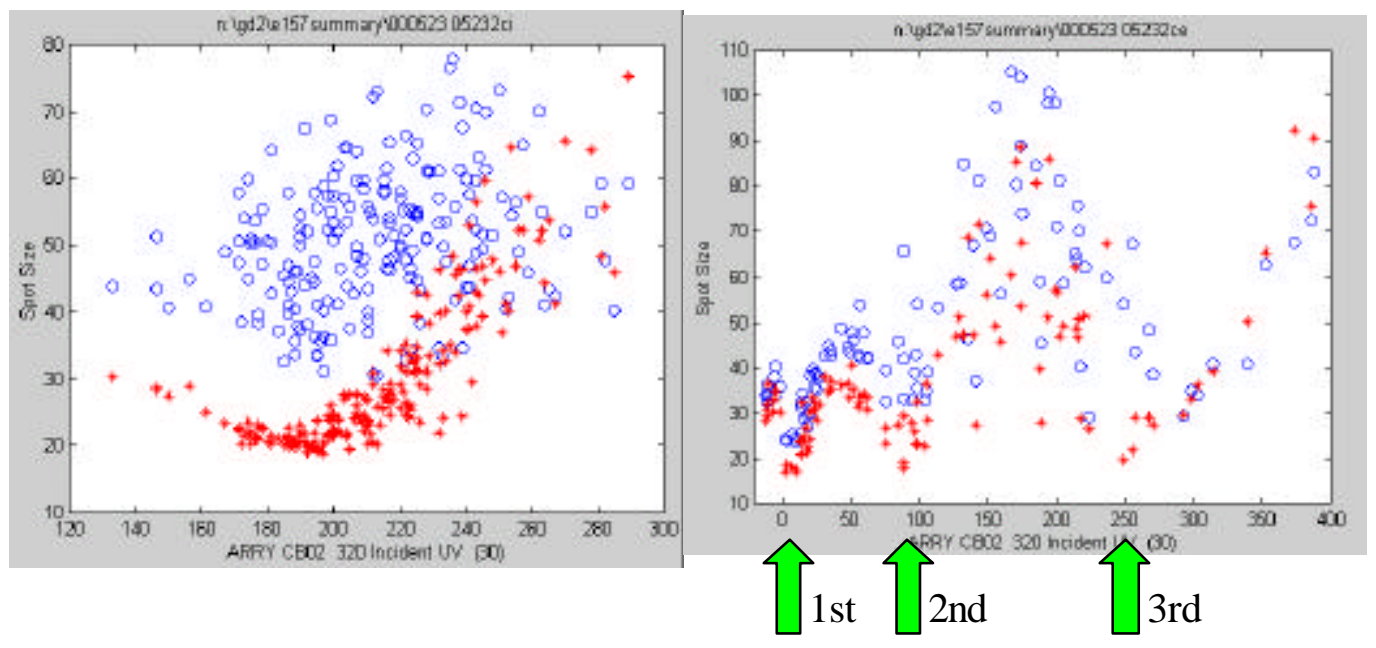

Figure 4.3.3 Justification of pinch location. The both images show the xRMS spot size (blue) and the yRMS spot size (red) at the aerogel vs. incident UV laser energy. The figure on the right shows that the $3^{\text {rd }}$ pinch is located around a GADC reading of 250 and that the pinch location is nearly the same for both the horizontal and vertical planes. The left image is figure 4.3.1 with the yRMS data superposed to show the pinch location.

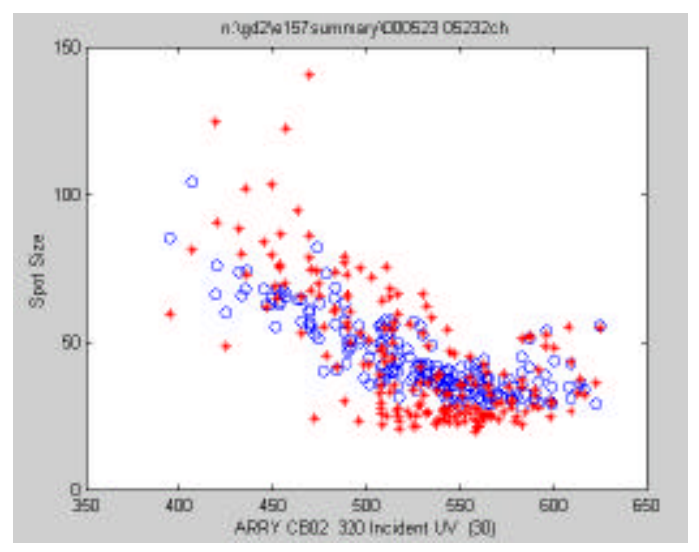

Figure 4.3.4 Fourth pinch data showing the location of the horizontal (blue) and vertical (red) pinches correspond to the same laser energy. 


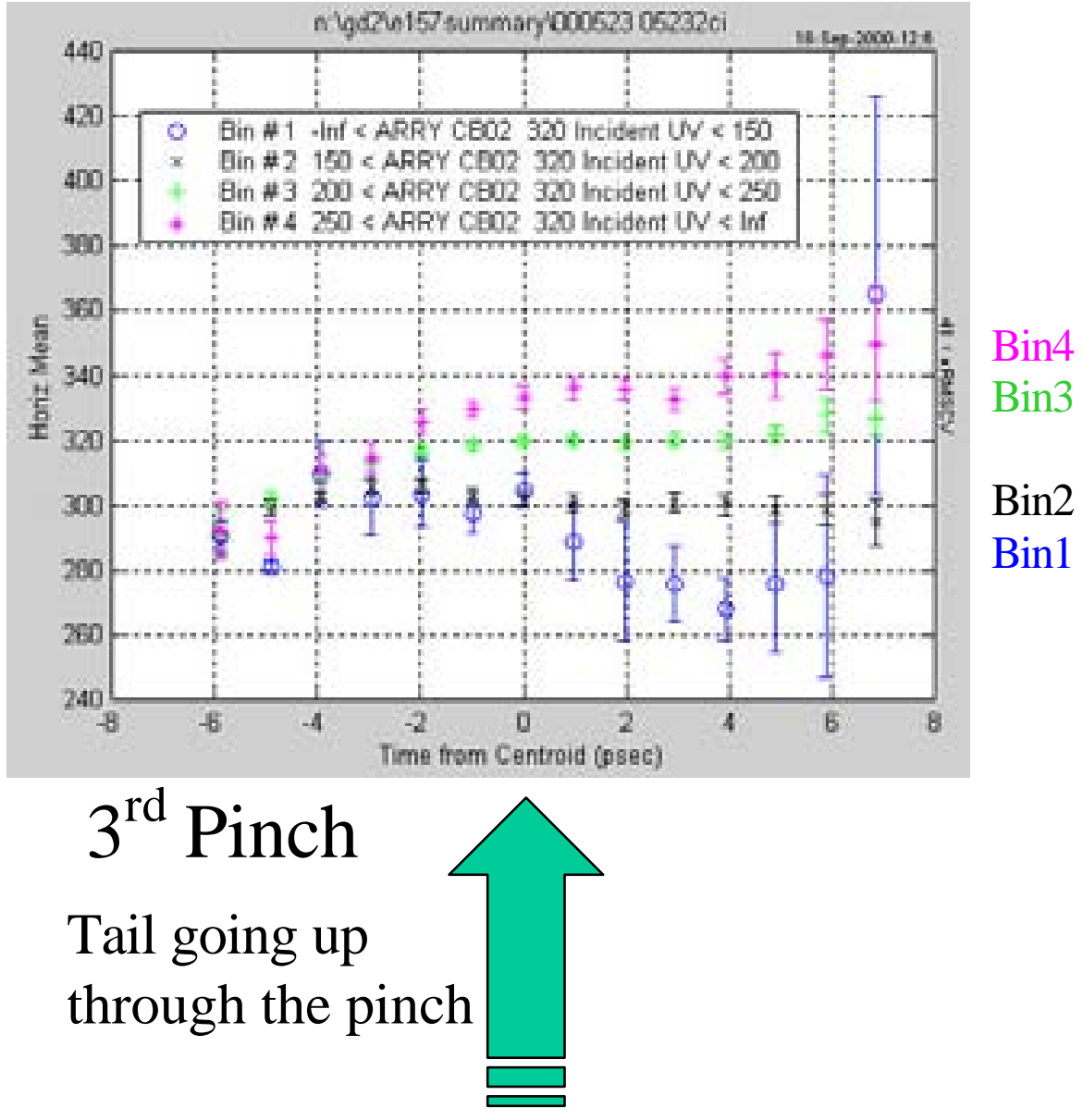

Figure 4.3.5 Tail motion at the $3^{\text {rd }}$ pinch with the pinch located between bins 2 and 3. $\left(\mathrm{N}=1.95 \times 10^{10} \mathrm{e}^{-} ; \mathrm{s}_{\mathrm{xUP}}=56 \mu \mathrm{m}\right)$

Nominal plasma density for the $3^{\text {rd }}$ pinch is about $1 \times 10^{14} \mathrm{~cm}^{-3}$. 


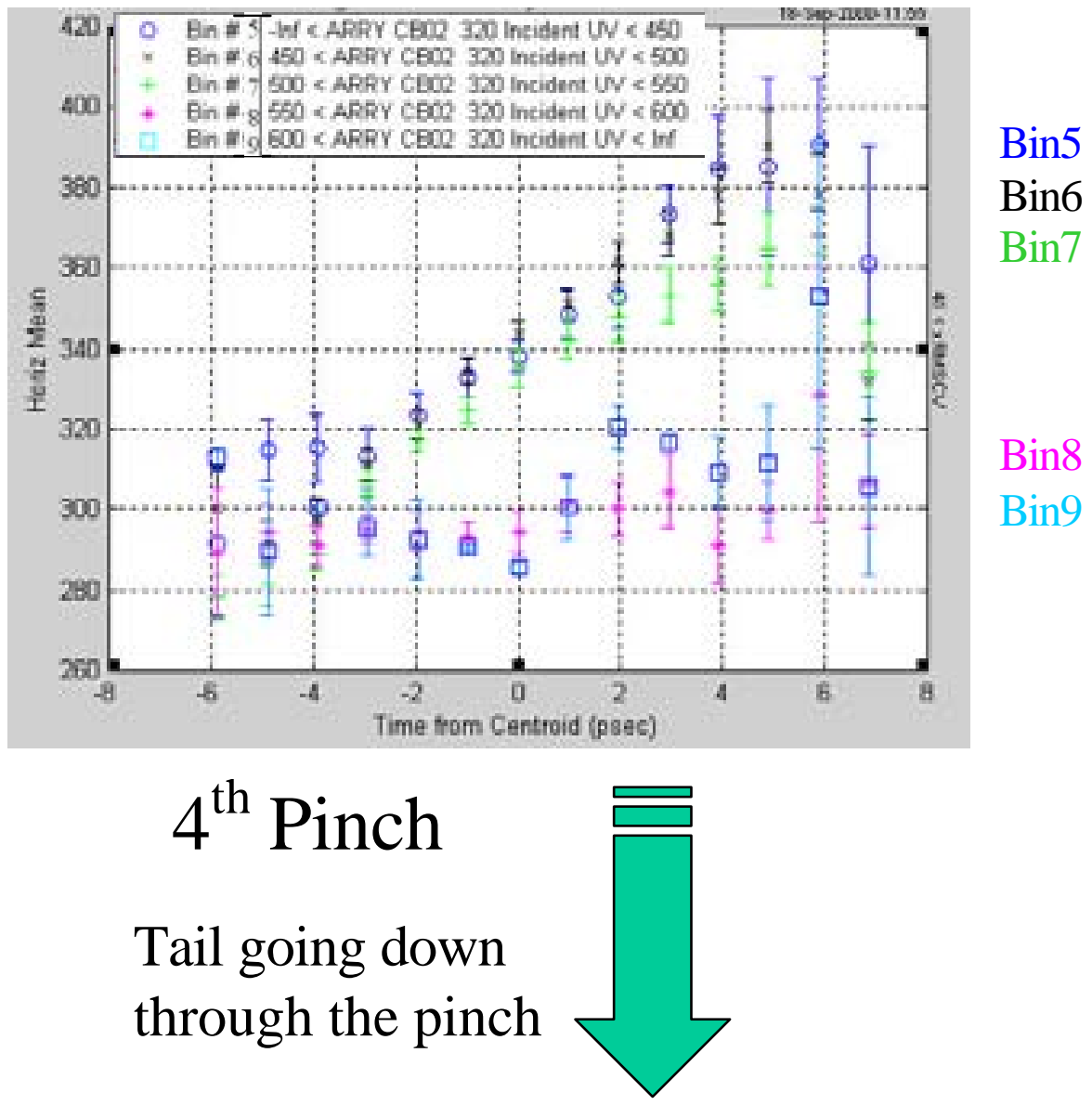

Figure 4.3.6 Tail motion at the $4^{\text {th }}$ pinch with the pinch located between bins 7 and 8. $\left(\mathrm{N}=1.95 \times 10^{10} \mathrm{e}^{-} ; \mathrm{s}_{\mathrm{xUP}}=56 \mu \mathrm{m}\right)$

Nominal plasma density for the $4^{\text {th }}$ pinch is about $2 \times 10^{14} \mathrm{~cm}^{-3}$. 


\subsection{Tail Growth on Streak Camera Diagnostic}

After showing that the beam is tilted and that this tilt results in tail flipping, we now want to determine if we are experiencing a hosing instability. We will do this by looking at the streak camera data for three cases. The first case will be laser off data. This will determine the initial tilt on the beam. The other two cases are at two different plasma densities; the second and fourth pinches at the Cherenkov detector. The second and fourth pinches were chosen because they are both an integer number of betatron oscillations and an integer number of tail oscillations. Only data that satisfied a transparency condition, where the beam spot size was equal to or slightly smaller than the laser off spot size, was chosen. The laser off spot size was $\sim 36$ pixels and the data cut at the $4^{\text {th }}$ pinch can be seen as the lower red bar in figure 4.3.2. The hypothesis is if an instability exists, then the magnitude of the head tail offset should rapidly grow with increasing plasma density (i.e. laser off ? $2^{\text {nd }}$ pinch ? $4^{\text {th }}$ pinch). Figures 4.4.1 shows the time slice analysis of the beam at the laser off, $2^{\text {nd }}$, and $4^{\text {th }}$ pinches respectively. The results show that there are not signs of significant growth up to the fourth pinch, the operating point of the experiment. 

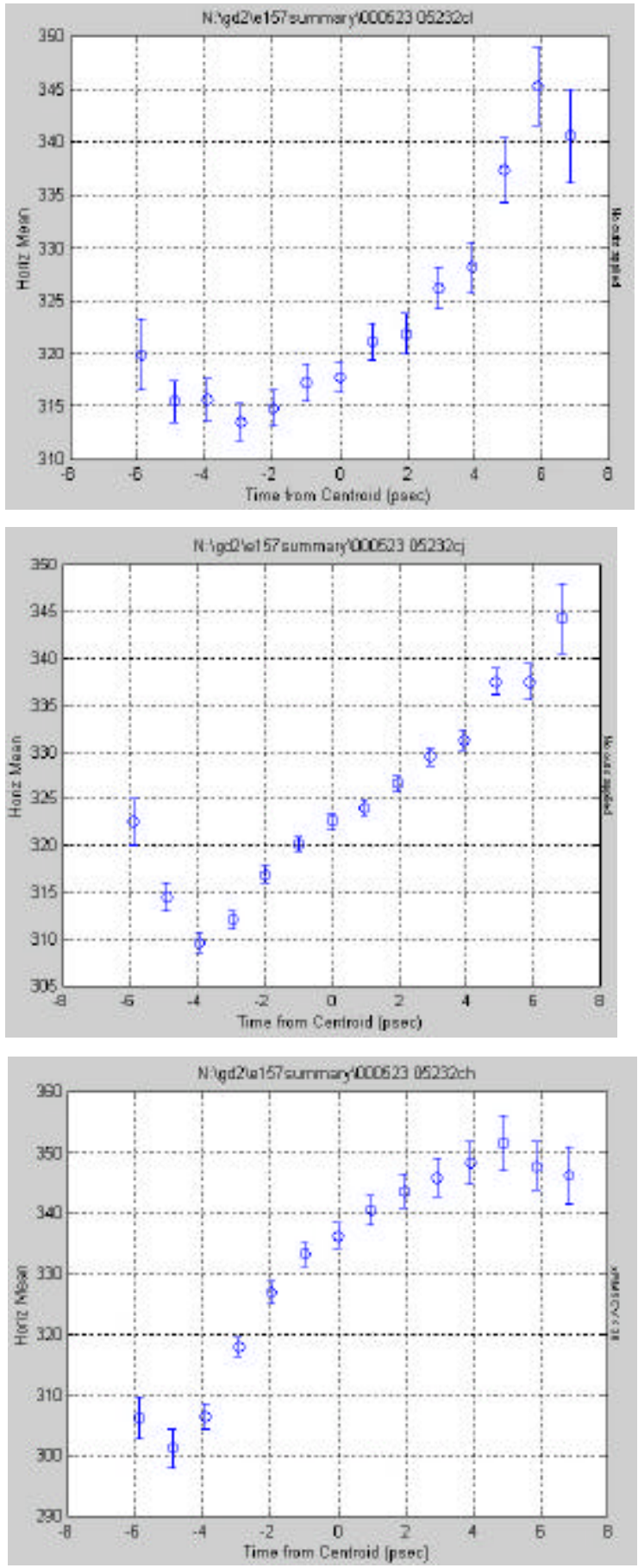

Figure 4.4.1 Time slice analysis at laser off, second, and fourth pinch
Laser Off

Second Pinch

$\mathrm{n}_{\mathrm{e}} \sim 0.3 \times 10^{14} \mathrm{~cm}^{-3}$.

Fourth Pinch

$\mathrm{n}_{\mathrm{e}} \sim 2 \times 10^{14} \mathrm{~cm}^{-3}$. 
Figure 4.4.2 shows the measured growth of the offset normalized to the initial offset (laser off) at the $4^{\text {th }}$ pinch. Both the initial and final offsets are measured with respect to the blow out time. In our case the blow out time was chosen to be 1 and 2 ps before the centroid. For the $2 \mathrm{ps}$ case, the mean position of the $-2 \mathrm{ps}$ slice in the fourth pinch data in figure 4.4.1 is 315 . The mean position of the slice $4 p s$ after the centroid is 328 . This implies the measured offset is 13 (328-315). The offset is calculated for each slice after blowout and plotted vs. a theoretical curve of a $140 \mathrm{~cm}$ long, $2 \times 10^{14} \mathrm{~cm}^{-3}$ plasma.

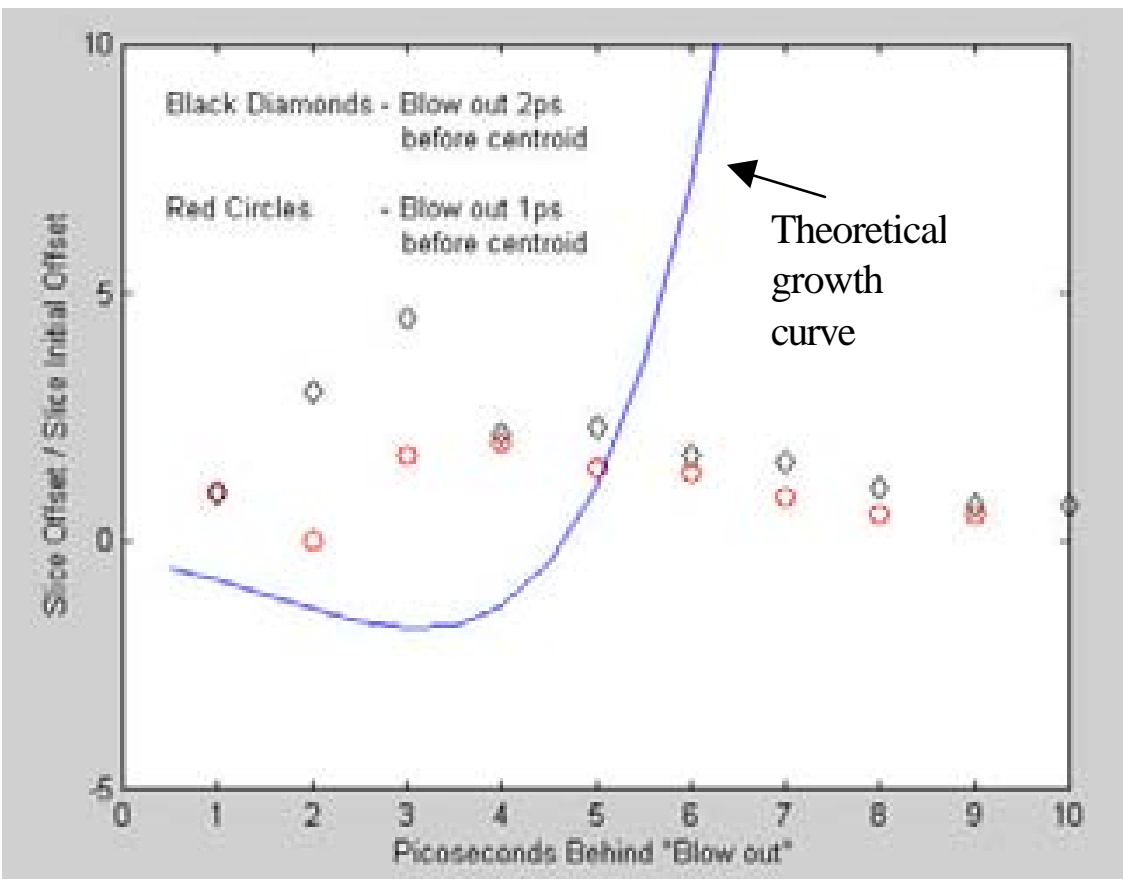

Figure 4.4.2 Growth of normalized slice offsets vs. theoretical hosing curve. $\left(\mathrm{N}=1.95 \times 10^{10} \mathrm{e}^{-} ; \mathrm{s}_{\mathrm{xUP}}=56 \mu \mathrm{m}\right)$ 


\subsection{BPM Data for a High Density Run}

One data run in the E157 experiment achieved a density higher than any other run. Whereas most runs could only achieve a density of $2 \times 10^{14} \mathrm{~cm}^{-3}$, one run achieved a density of approximately $6 \times 10^{14} \mathrm{~cm}^{-3}$. This is important because the hosing growth rate increases rapidly with increasing density. Because this run was energy scan data, the centroid motion experienced large displacements. These kicks were large enough to steer the beam off of the streak camera slit, rendering the streak camera data unusable. This mandated the use of BPMs to look for the hosing instability.

To analyze this data, we follow the oscillations of the beam exiting the plasma as measured by the downstream OTR and the beam position monitors 6130, 6160, 6167, and 6170 (figure 4.5.1). For each shot, the deviation from laser off position is calculated and this deviation is plotted versus the BPM's (or downstream OTR) position relative to the exit of the plasma. Figure 4.5.2 shows typical beam trajectory leaving the plasma. A straight line fit is made to the five points (a straight line indicates that upon leaving the plasma, the beam centroid travels ballistically), and the slope of the exit trajectory is found. Figure 4.5.3 shows a plot of the slope of the beam's exit trajectory versus incident laser energy for all 200 shots. 

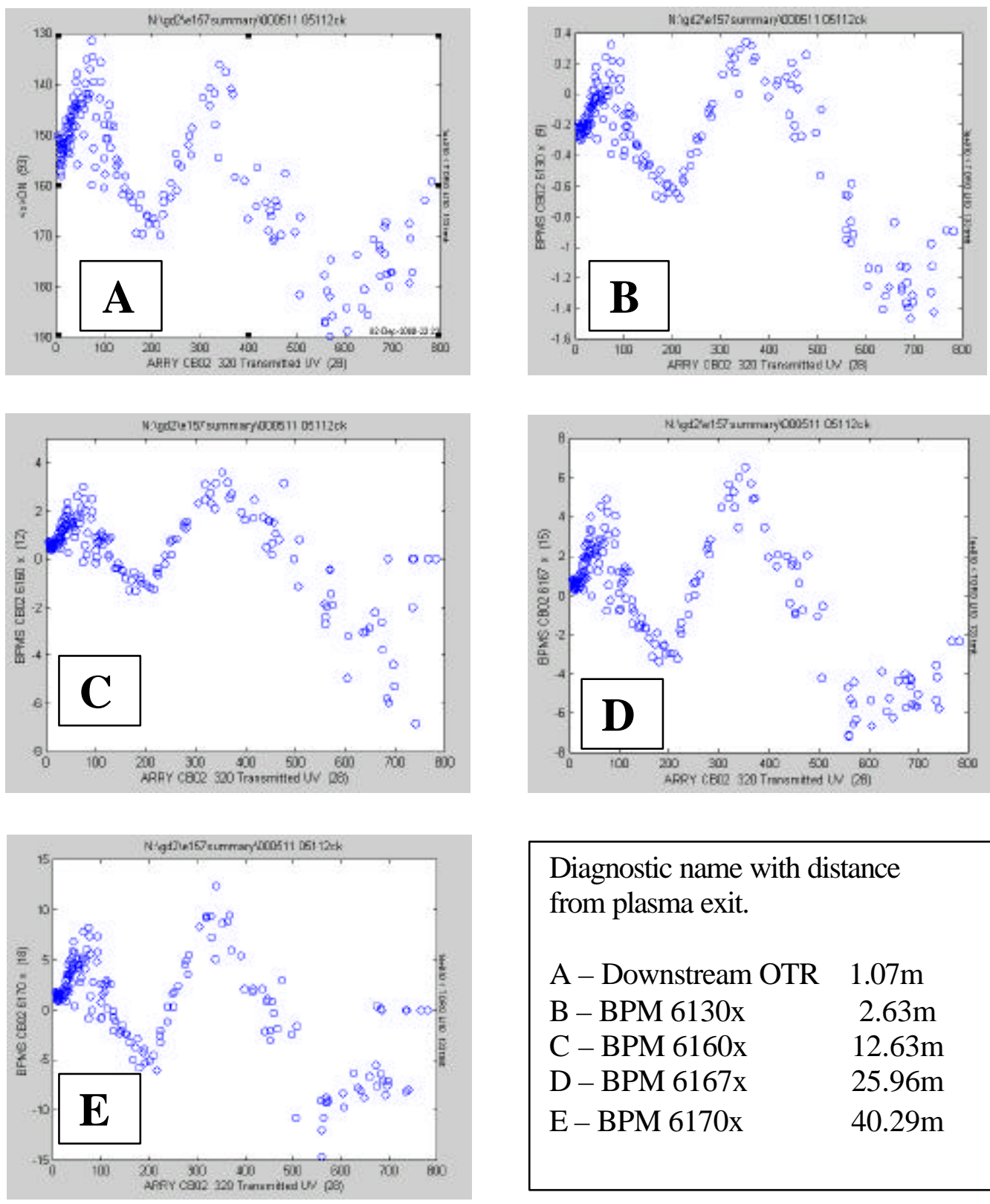

Diagnostic name with distance from plasma exit.

$\begin{array}{lc}\text { A - Downstream OTR } & 1.07 \mathrm{~m} \\ \text { B - BPM 6130x } & 2.63 \mathrm{~m} \\ \text { C - BPM 6160x } & 12.63 \mathrm{~m} \\ \text { D - BPM 6167x } & 25.96 \mathrm{~m} \\ \text { E-BPM 6170x } & 40.29 \mathrm{~m}\end{array}$

Figure 4.5.1 Oscillations of beam centroid after plasma exit as measured on downstream OTR and on BPMs 6130, 6160, 6167, and 6170. The x-axis is the incident UV laser energy and the $y$-axis is the transverse position of the beam centroid. $\left(\mathrm{N}=2 \times 10^{10} \mathrm{e}^{-} ; \mathrm{s}_{\mathrm{xUP}}=36 \mu \mathrm{m}\right)$ 


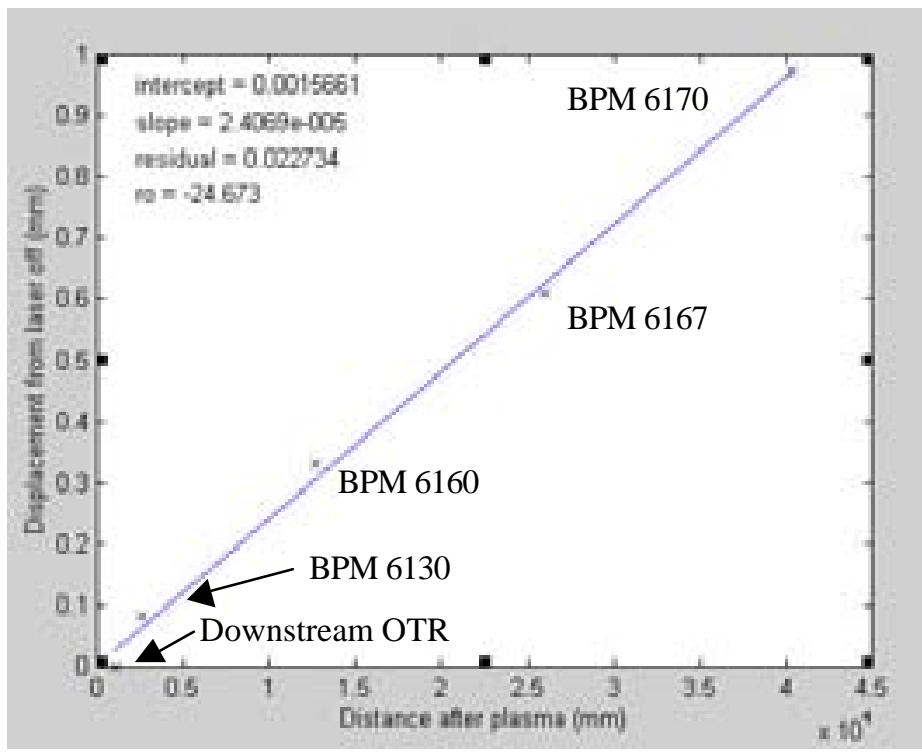

Figure 4.5.2 Beam trajectory exiting plasma

$$
\left(\mathrm{N}=2 \times 10^{10} \mathrm{e}^{-} ; \mathrm{s}_{\mathrm{xUP}}=36 \mu \mathrm{m}\right)
$$

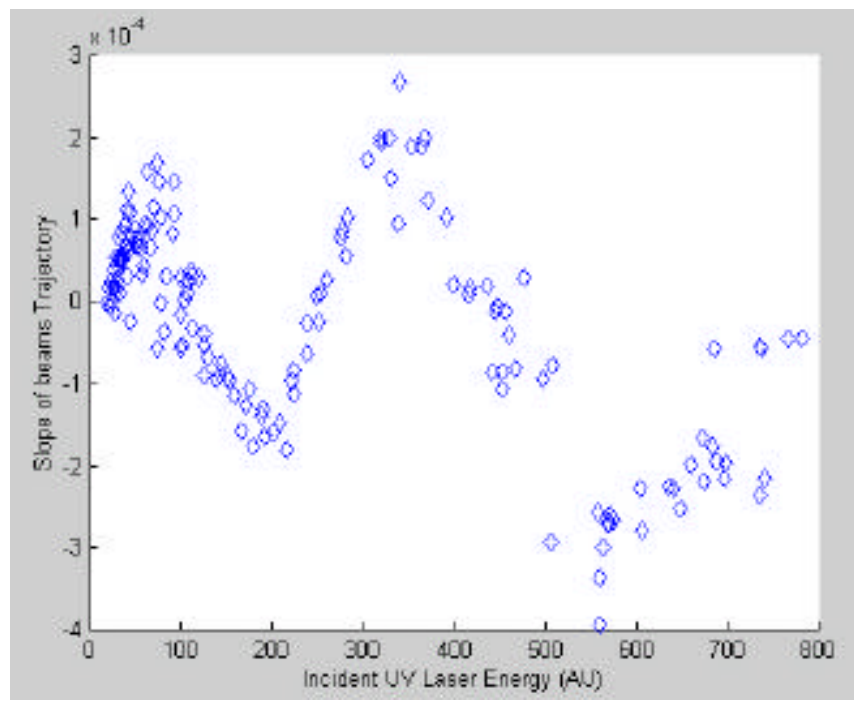

Figure 4.5.3 Slope of the beams exit trajectory as a function of laser energy (plasma density) $\left(\mathrm{N}=2 \times 10^{10} \mathrm{e}^{-} ; \mathrm{s}_{\mathrm{xUP}}=36 \mu \mathrm{m}\right)$ 
Figure 4.5.3 show the slope versus laser energy, but it is more insightful to convert the incident laser energy into plasma density. This is done by fitting the beam envelope equation (4.1.1) to the betatron oscillation data (i.e. spot size fluctuations) as shown in figure 4.5.4.

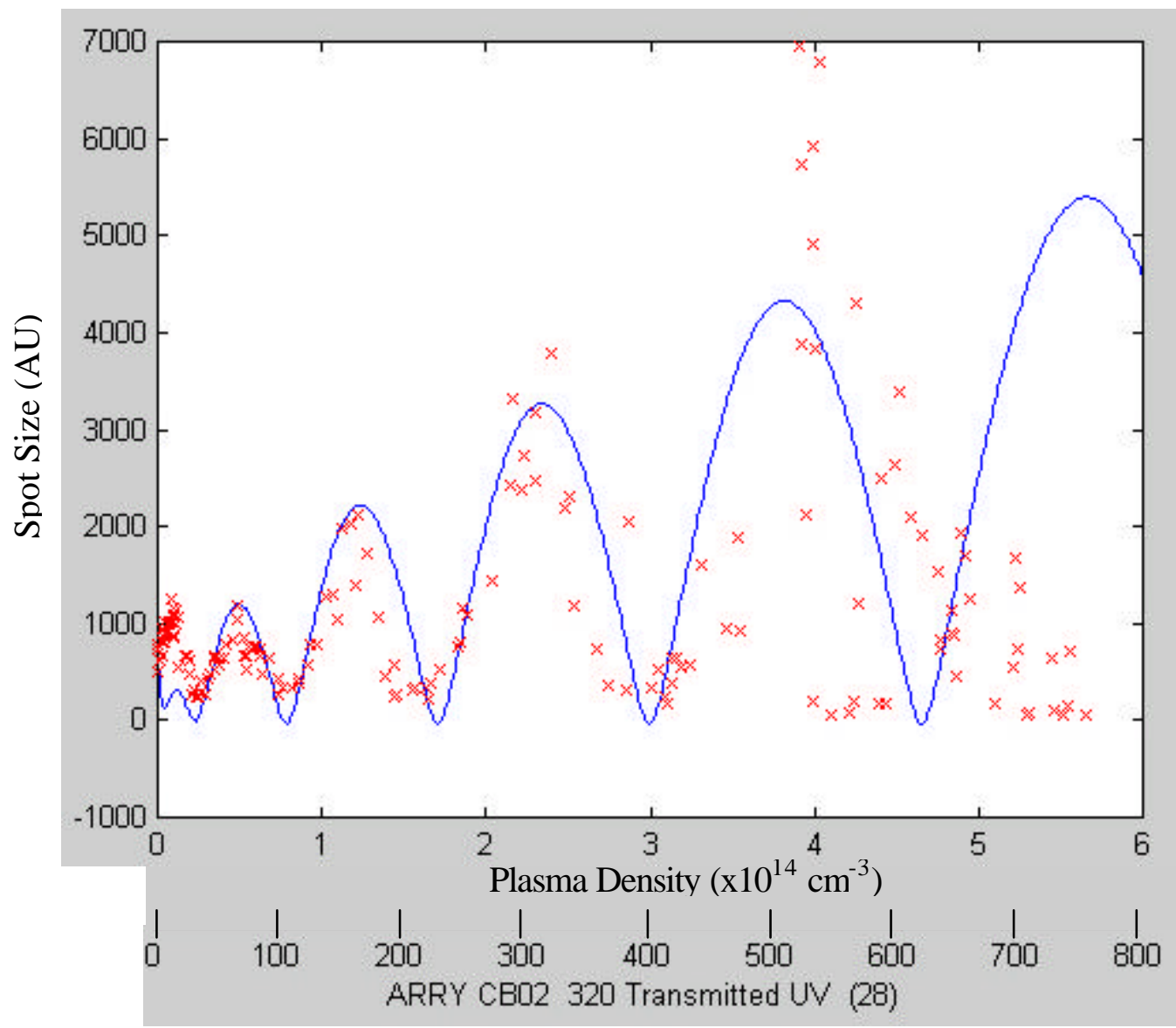

Figure 4.5.4 Plot of envelope equation (shown in blue) versus plasma density superimposed on a plot of the beams spot size (red diamonds) versus incident UV laser energy. This fit is used to convert the incident UV laser energy into plasma density. 
The equation for the exit trajectories slope in the absence of hosing (2.1.17) has four variables: the slope, $\mathrm{r}$; the initial offset, $\mathrm{r}_{\mathrm{o}}$; the plasma density, $\mathrm{k}_{\beta}$; and the plasma column length, s. The only unknown is the initial offset. By applying the formula for centroid oscillations with no growth (2.1.17) and solving for $r_{o}$ at low densities, we find that the initial offset is about $24 \mu \mathrm{m}$.

The slope is the ratio of the beams transverse energy to its longitudinal energy.

$$
r^{\prime}=\frac{m_{e} c^{2} \gamma_{\perp}}{m_{e} c^{2} \gamma_{\|}}=\frac{\gamma_{\perp}}{\gamma_{\|}}
$$

Figure 4.5.2 shows the calculated perpendicular energy of the beam (found from the slope trajectory) plotted vs. plasma density. The blue diamonds represent calculated values and the black x's represents a theoretical envelope (neglecting the sinusoidal term for clarity) curve of perpendicular energy in absence of hosing .

$$
E_{\perp}=r_{o} k_{\beta} m c^{2}
$$

The red circles represent a theoretical envelope (neglecting the sinusoidal term for clarity) curve for perpendicular energy with hosing.

$$
E_{\perp}=0.341 \frac{e^{A}}{A^{3 / 2}} r_{o} k_{\beta} m c^{2}
$$

The slice index $\mathrm{t}$ was chosen to be $1.25 \mathrm{ps}$ so that the theoretical curve matched a data point at the plasma density of $4 * 10^{14} \mathrm{~cm}^{-3}$. A small value of $\mathrm{t}$ is expected since "blow out" occurs near the centroid and BPMs measure the position of the centroid. 


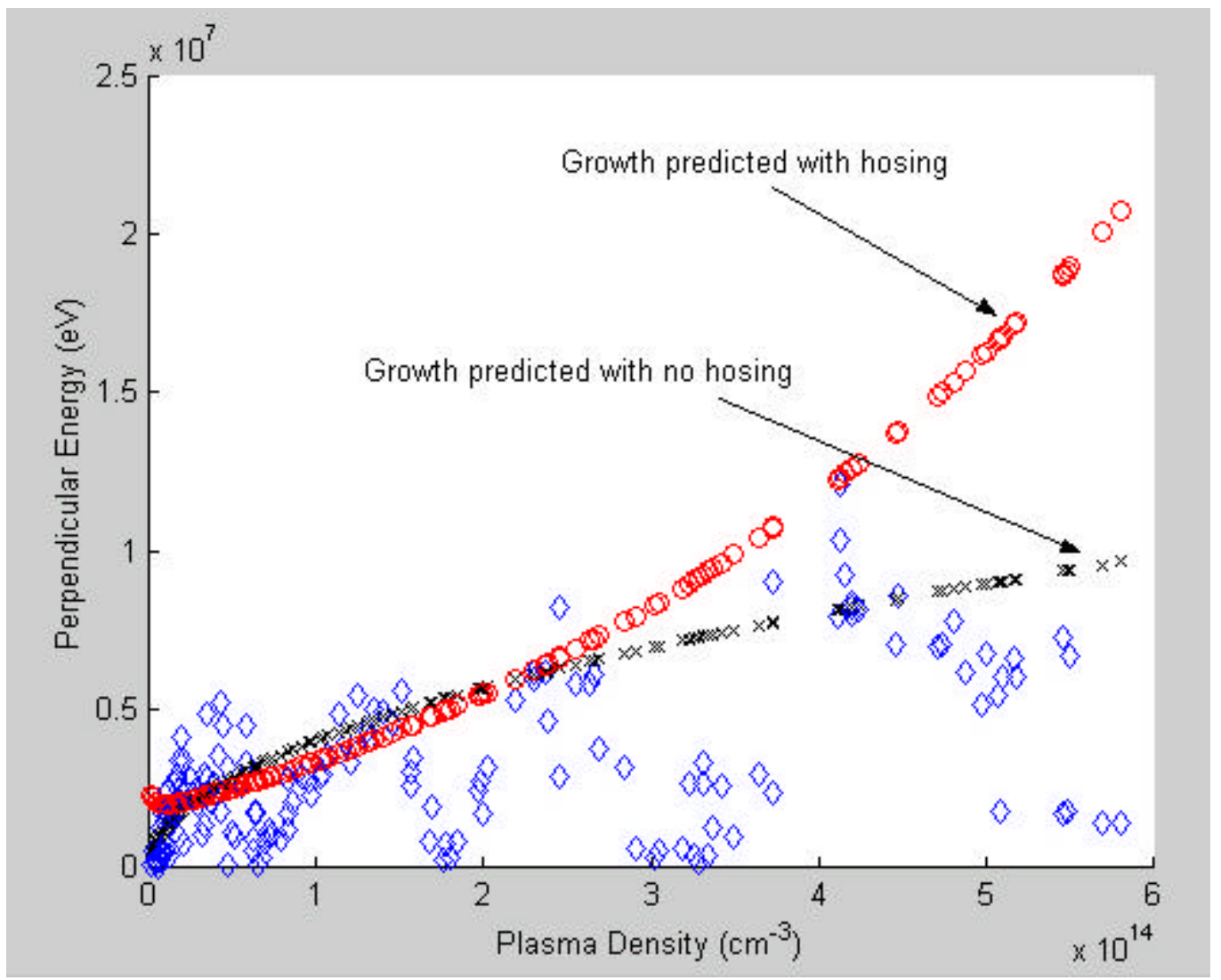

Figure 4.5.2 Perpendicular Energy of Beam in a High Density Plasma $\left(\mathrm{N}=2 \times 10^{10} \mathrm{e}^{-} ; \mathrm{s}_{\mathrm{xUP}}=36 \mu \mathrm{m}\right)$

The large discrepancy at the low plasma density is due to beam jitter and a divide by zero numerical error. We also know from the time slice analysis that hosing is not present at these low densities. On the other hand, a few of the data points around $4 \times 10^{14} \mathrm{~cm}^{-3}$ show evidence of growth on the order of $1.5 \mathrm{x}$. This value is consistent with the growth factor predicted by the tilted beam hosing equation 2.2 .5 for a $\mathrm{t}=$ $1.25 \mathrm{ps}$ 


\section{Conclusion}

\subsection{Summary}

A subset of the transverse dynamics of an ultra-relativistic electron beam propagating through a meter long plasma has been experimentally measured. The electron hosing instability arises when electrons are offset in an ion column. In a single bunch PWFA experiment, a beam tilt will result in offset electrons. Time-slice analysis of the electron bunch has quantified a head-tail offset (i.e. tilt). Further analysis has shown the tail off the beam oscillating inside the plasma. Finally, the magnitude of the oscillation was not observed to significantly grow up to the operating plasma density of the E157 experiment. In other words, there is no evidence of the electron hosing instability at the operating parameters of the E157 experiment. Recent simulations by E. Dodd corroborate the above statement [28]. There is possible evidence of the hosing instability in a single high-density plasma run, but further data is needed at such a high density to be conclusive.

\subsection{Future Directions}

An upcoming experiment at SLAC will provide the opportunity to expand on these results [29]. First, an imaging spectrometer will be constructed that will image the beam from the output of the plasma onto the aerogel. This will allow us to analyze the beam at the exit of the plasma as opposed to analyzing it after 12 meters of drift. 
A second benefit will be the analysis of positron beams. In an analogous situation to ion channel formation with electron beams, positron beams will create a channel where electrons rush in. The transverse dynamics of a positron beam will be rich with physics that will be both challenging and rewarding to analyze. 


\section{Appendix A FFTB beamline}

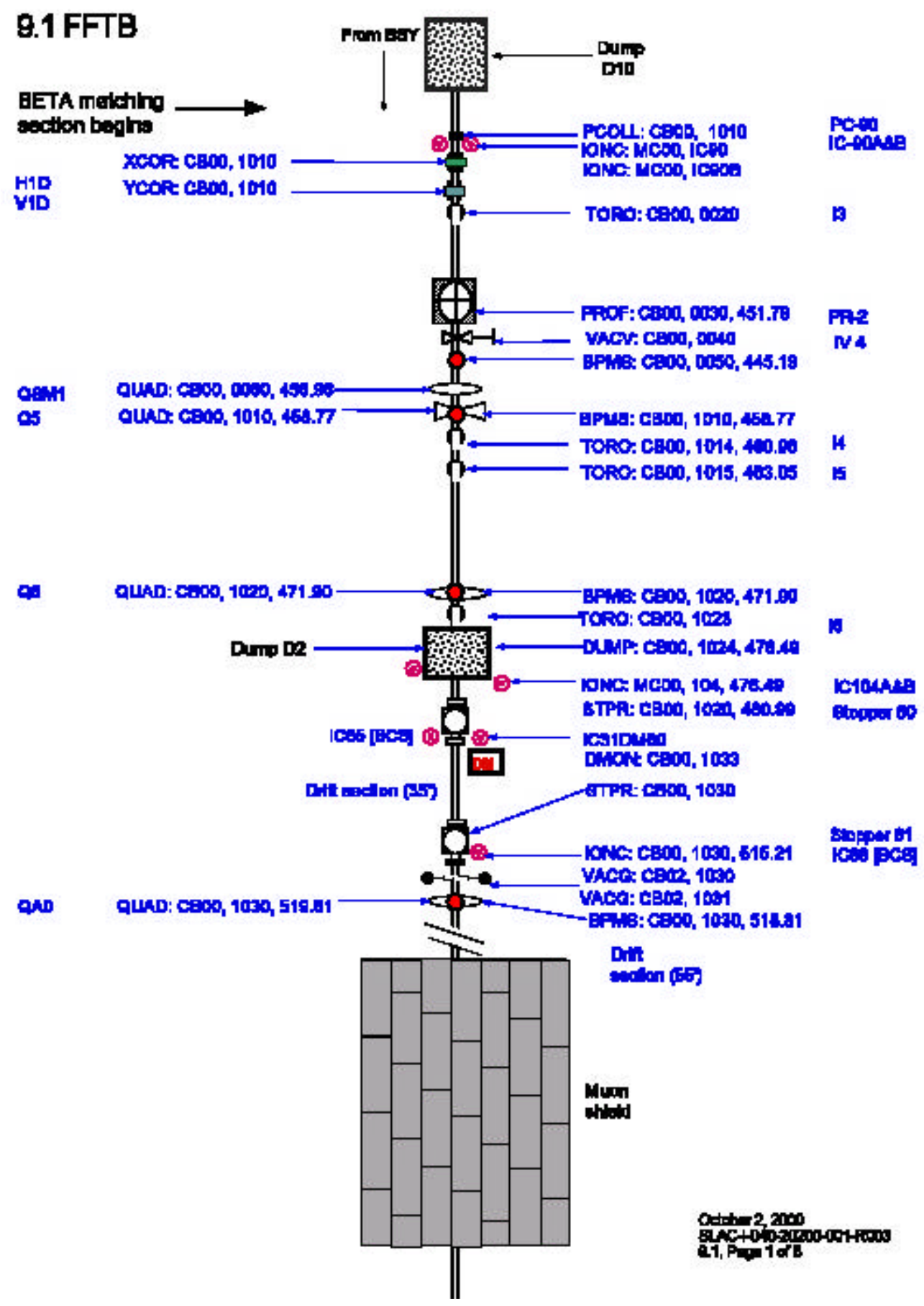




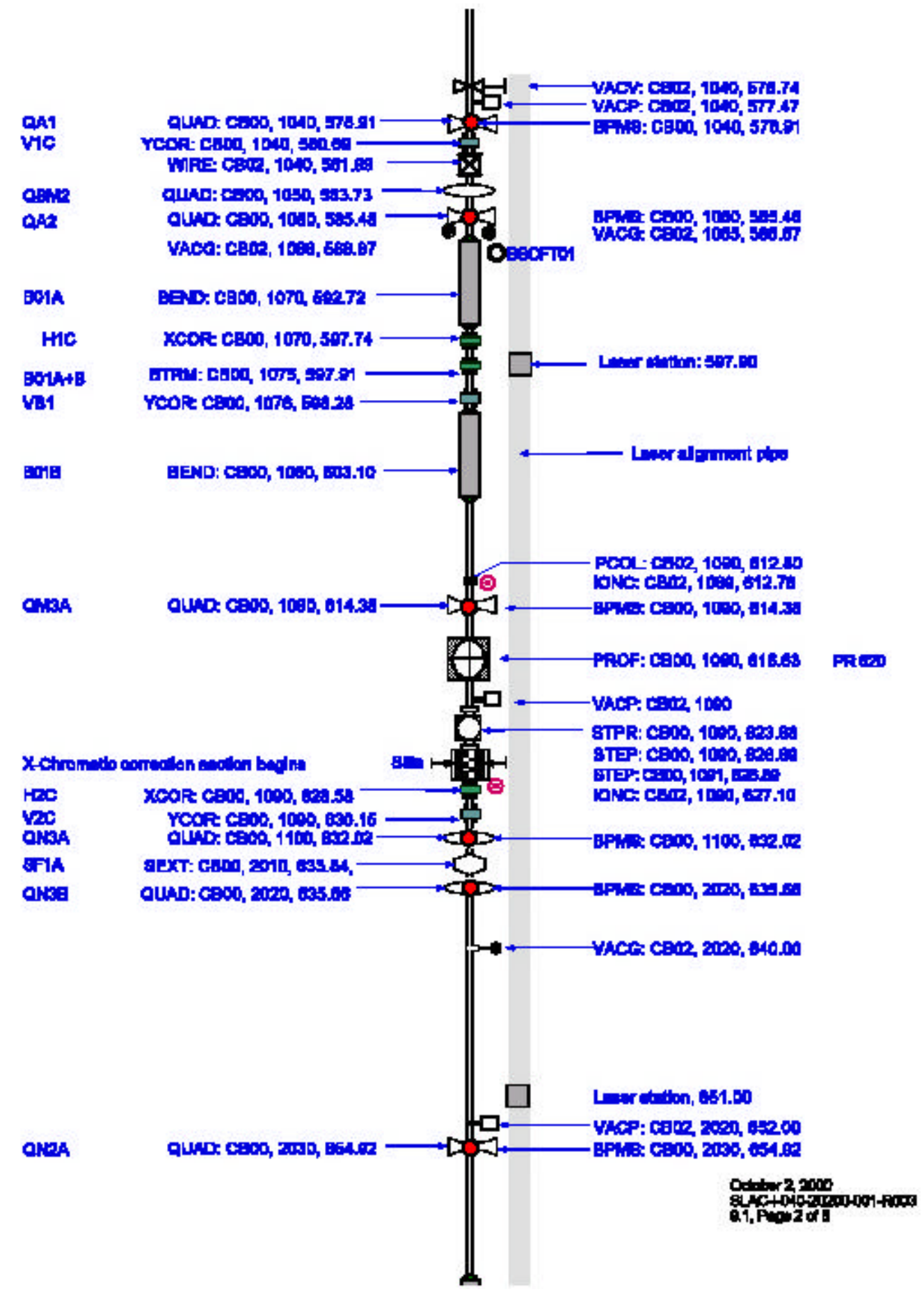




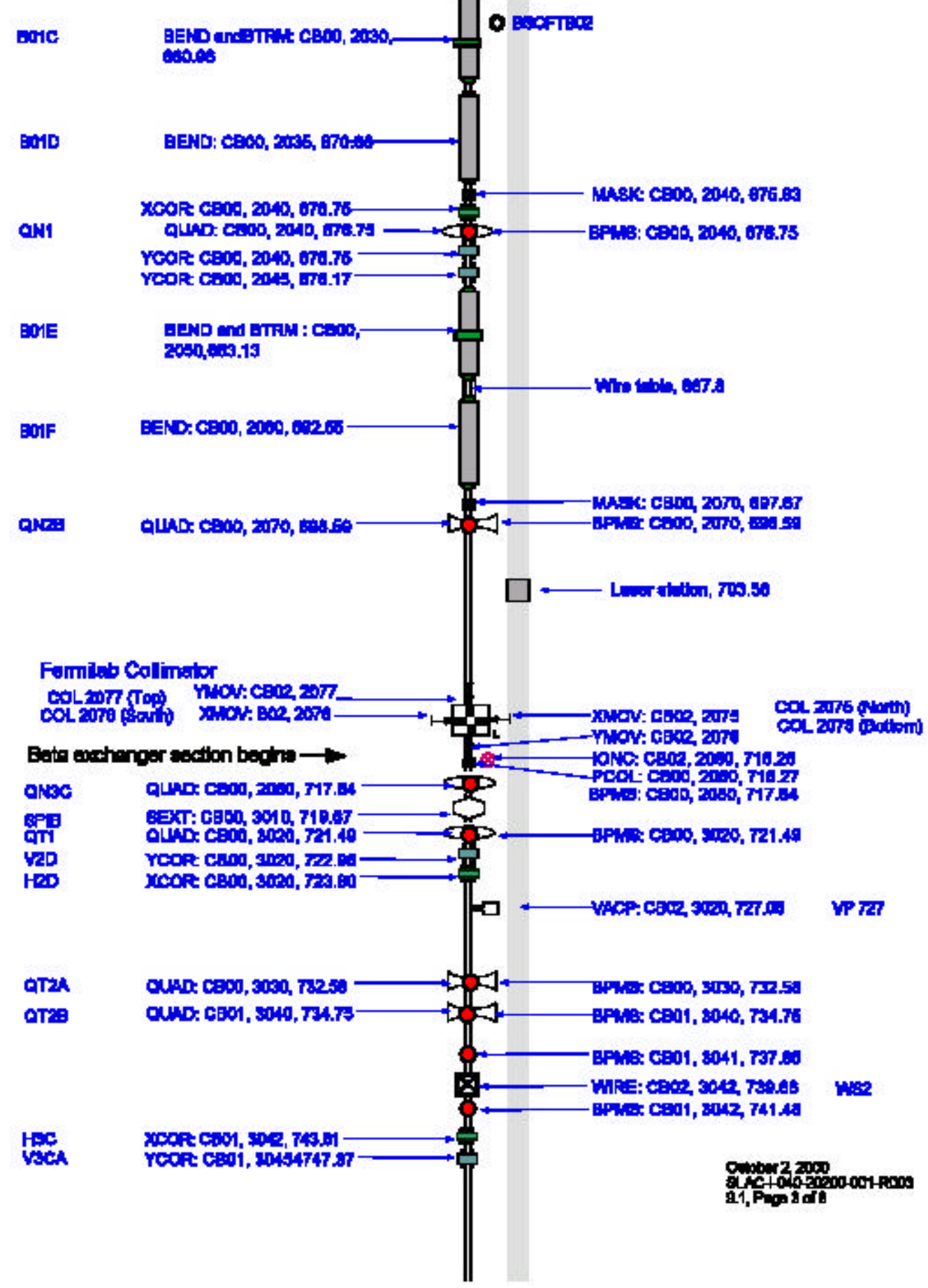




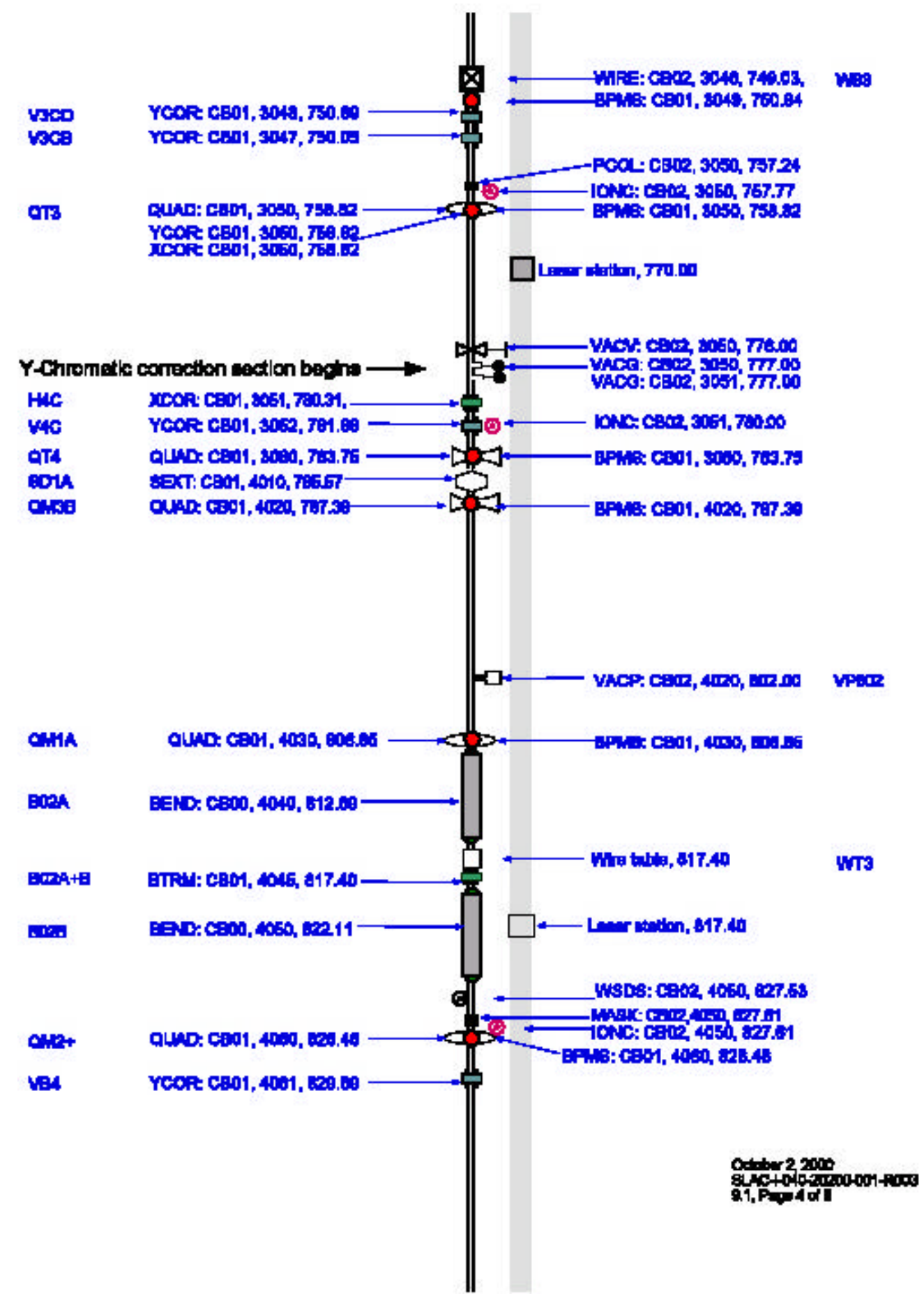




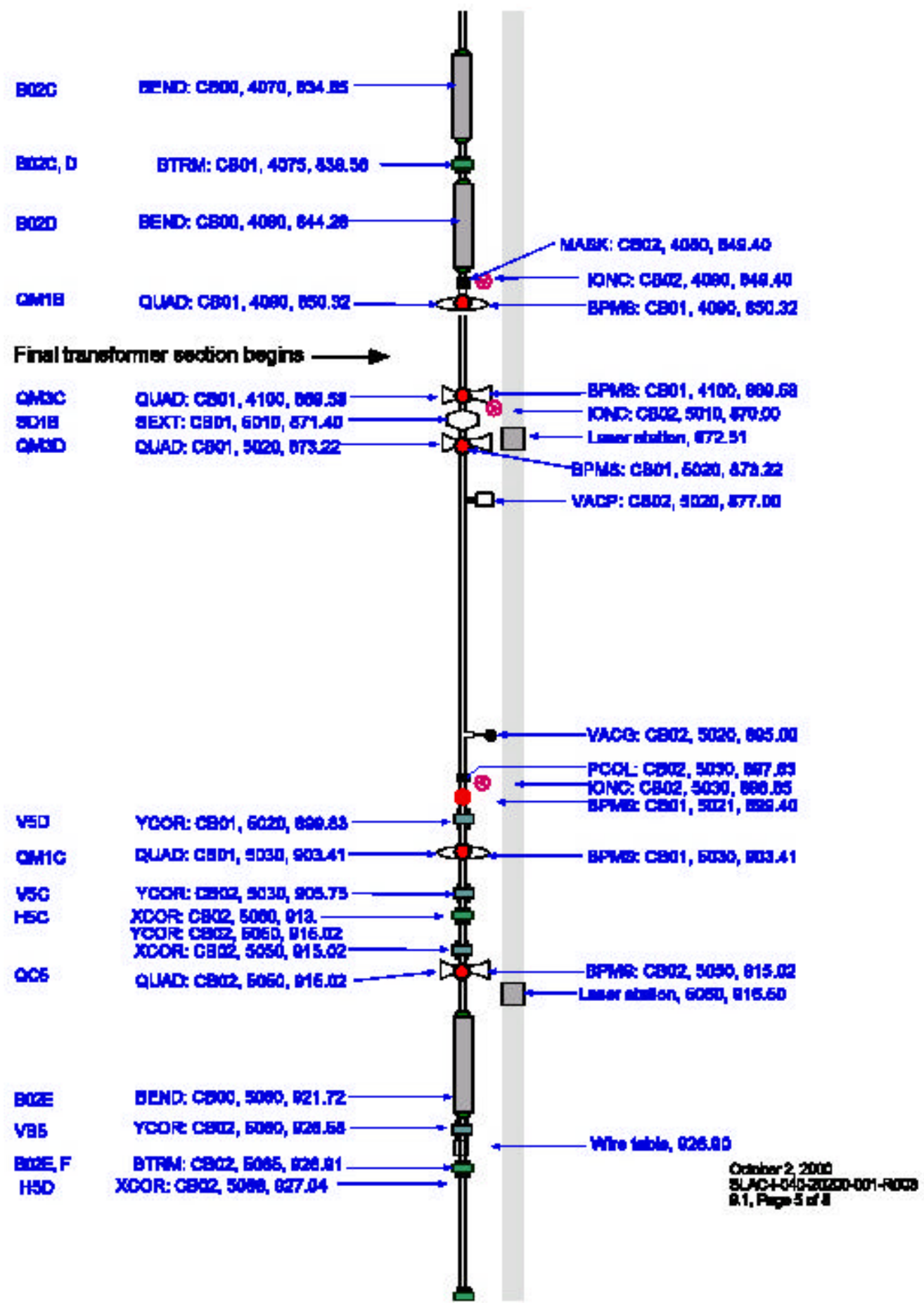




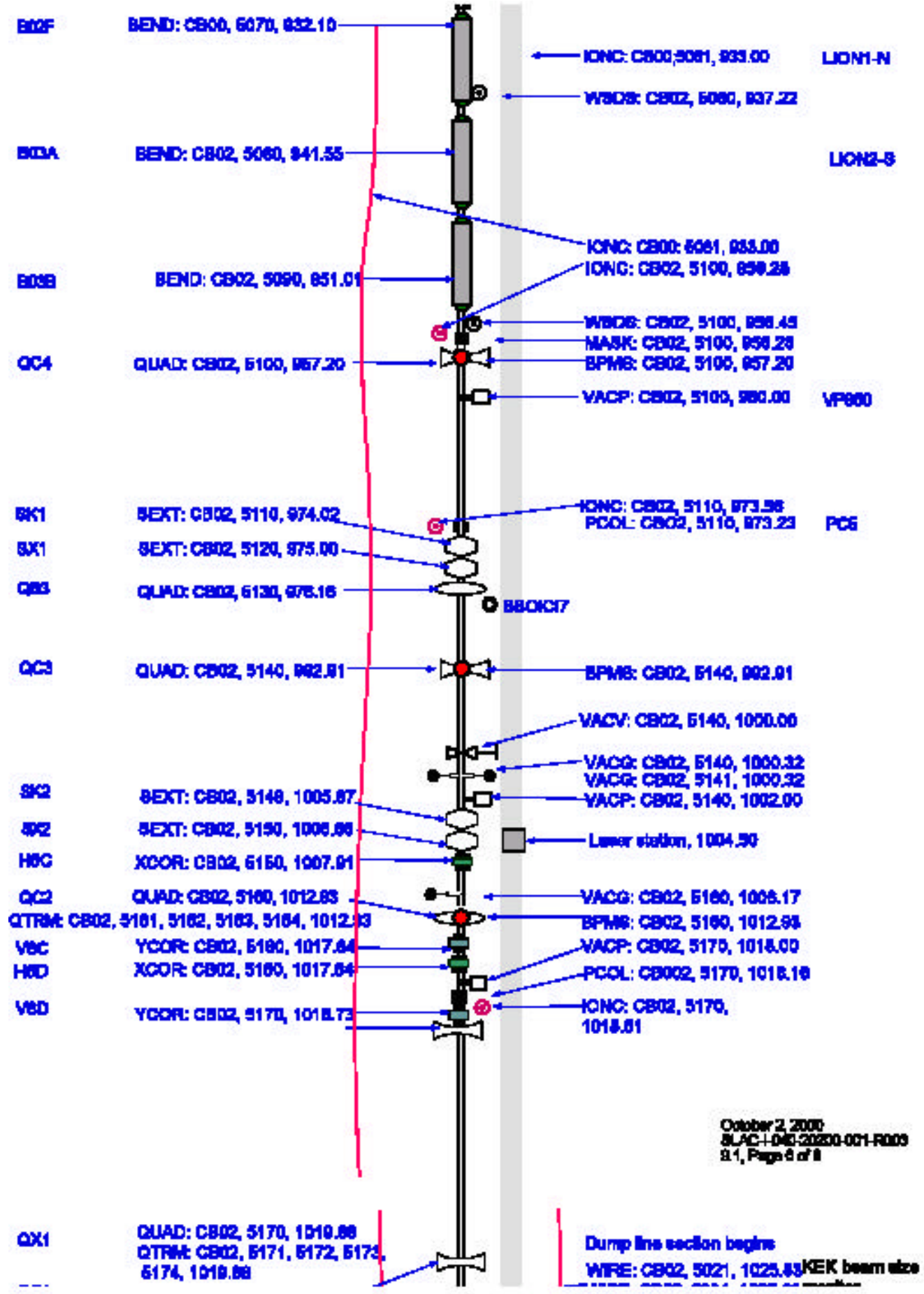




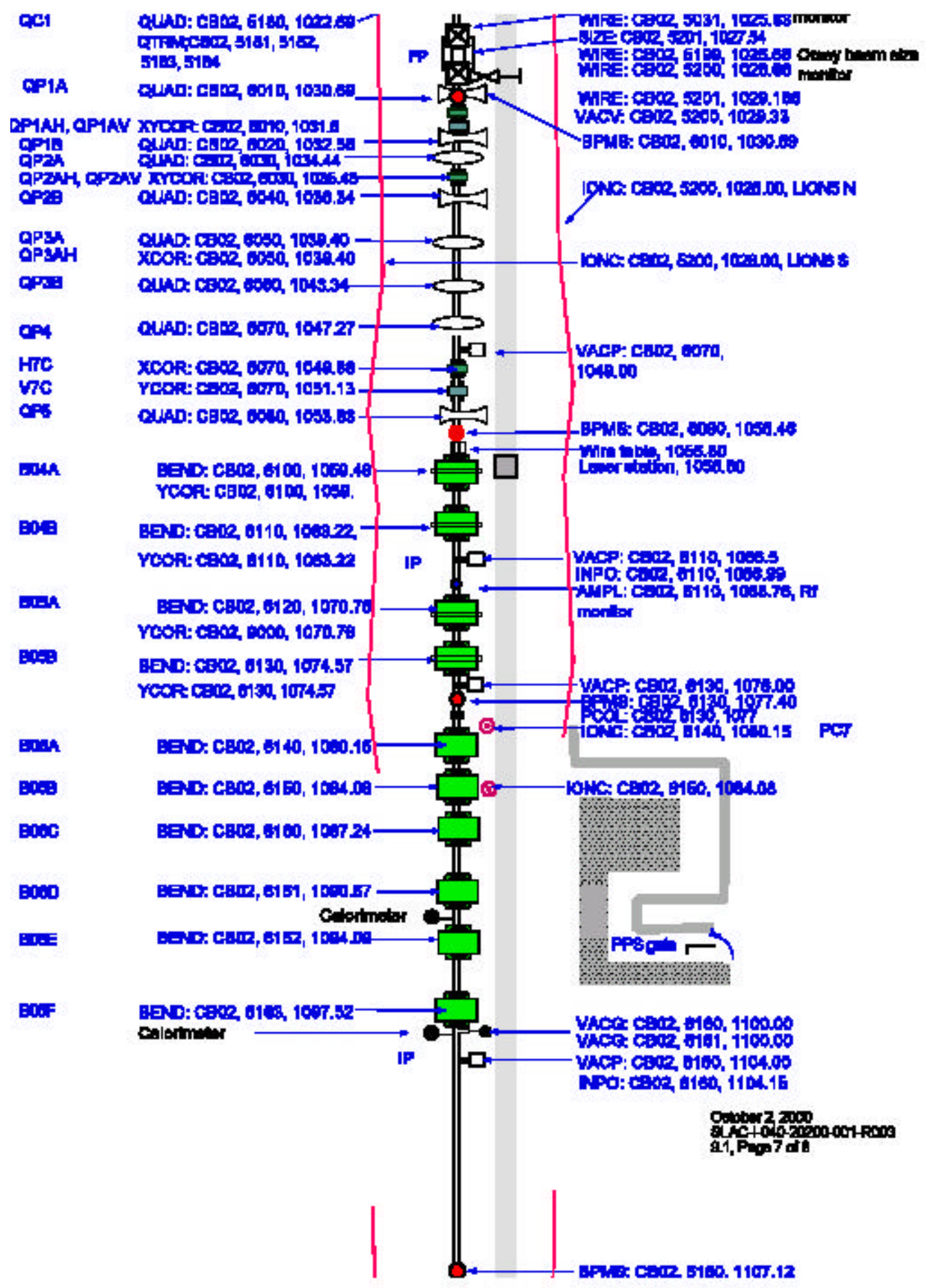




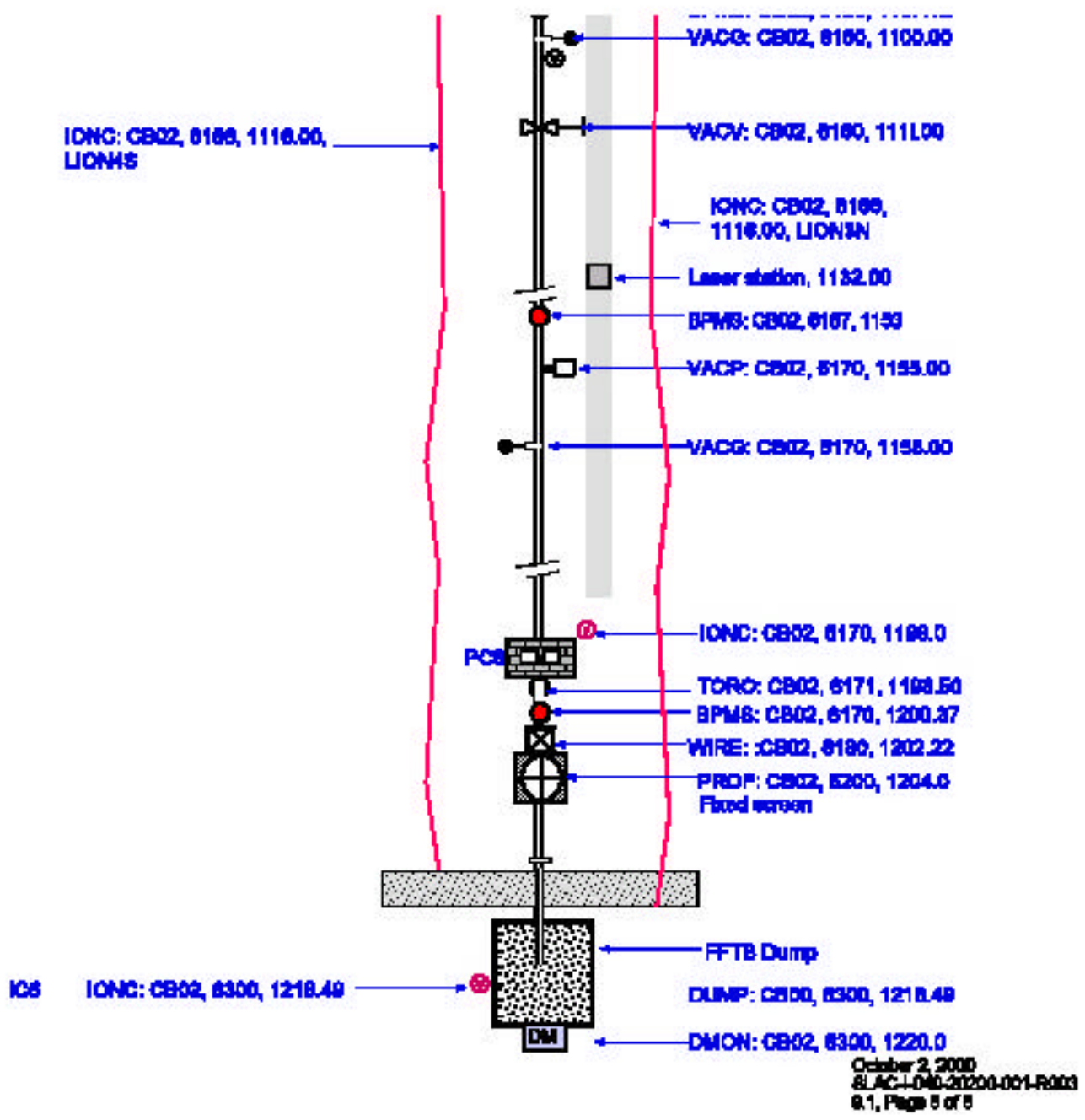




\section{Appendix B Typical Electron Beam Parameters}

Number of electrons per bunch

Bunch Energy

Gamma

Bunch Radius

Bunch Length

Normalized Emittance x

Normalized Emittance y
$1.8-2.0 \times 10^{10}$

$28.5 \mathrm{GeV}$

55773

$50 \mu \mathrm{m}$

$0.7 \mathrm{~mm}$

$2.3 \mathrm{ps}$

$5 \times 10^{-5} \mathrm{~m} \mathrm{rad}$

$0.5 \times 10^{-5} \mathrm{~m} \mathrm{rad}$ 


\section{Bibliography}

[1] J.M. Dawson, "Nonlinear electron oscillations in a cold plasma," Phys. Rev., vol. 133, pp. 383-387, 1959.

[2] E. Esarey, P. Sprangle, J. Krall et al, "Overview of plasma based accelerator concepts,” IEEE Transactions on Plasma Science, 24, 252 (1996).

[3] C.E. Clayton, K.A. Marsh, A. Dyson, M. Everett, A. Lal, W.P. Leemans, R. Williams, and C. Joshi, "Ultrahigh-gradient acceleration on injected electrons by laser-excited relativistic electron plasma waves," Phys. Rev. Lett. vol. 70, pp. 37-40, 1993.

[4] W. B. Mori, "Computer simulations on the acceleration of electrons by fast large-amplitude plasma waves driven by laser beams," Master's Thesis, Univ. California Los Angeles, 1984.

[5] K. Nakajima, D. Fisher, T. Kawakubo, H. Nakanishi, A. Ogata, Y. Kato, Y. Kitagawa, R. Kodama, K. Mima, H. Shiraga, K. Suzuki, K. Yamakawa, T. Zhang, Y. Sakawa, T. Shoji, Y. Nishida, N. Yugami, M. Downer, and T. Tajima, "Observation of ultrahigh gradient electron acceleration by a selfmodulated intense short laser pulse,” Phys. Rev. Lett. vol. 74, pp. 4428-4431, 1995.

[6] Katsouleas, T.; Lee, S.; Chattopadhyay, S.; Leemans, W.; Assmann, R.; Chen, P.; Decker, F.J.; Iverson, R.; Kotseroglou, T.; Raimondi, P.; Raubenheimer, T.; Eokni, S.; Siemann, R.H.; Walz, D.; Whittum, D.; Clayton, C.; Joshi, C.; Marsh, K.; Mori, W.; Wang, G., " A proposal for a $1 \mathrm{GeV}$ plasma-wakefield acceleration experiment at SLAC," Proceedings of the 1997 Particle Accelerator Conference, Vancouver, BC, Canada, 12-16 May 1997. Piscataway, NJ, USA: IEEE, 1998. p.687-9 vol.1

[7] T.O. Raubenheimer et al, "Zeroth order design report for the next linear collider,” SLAC-Report-474 May 1996

[8] J.J. Su, T. Katsouleas, J.M. Dawson, and R. Fedele, "Plasma lenses for focusing particle beams," Phys. Rev. A., vol. 41. pp. 3321, 1990.

[9] P. Chen, K. Oide, A.M. Sessler, and S.S. Yu, "Plasma based adiabatic focuser," Phys. Rev. Lett., vol. 64, pp. 1231, 1990. 
[10] W.A. Barletta, "Linear emittance damper with megagauss fields," Proceedings of the Workshop on New Developments in Particle Acceleration Techniques, (CERN Service d'Information, Orsay), pp. 544, 1987.

[11] K. Takayama, S. Hiramatsu, "Ion-channel guiding in a steady-state freeelectron laser," Phys. Rev. A, vol. 37, pp. 173, 1988.

[12] S. Hiramatsu et al, "Proposal for an X-band single-stage FEL," Nucl. Instrum. Methods Phys. Res., Sec. A., vol. 285, pp. 83, 1989

[13] K.J. O'Brien, "Theory of the ion-hose instability," Journal of Applied Physics, vol.65, (no.1), 1 Jan. p.9-16, 1989.

[14] K.T. Nguyen et al, "Transverse instability of an electron beam in beam induced ion channel," Applied Physics Letters, vol.50, (no.5), 2 Feb.. pp. 239-41, 1987.

[15] K.J. O'Brien et al, "Experimental observation of ion hose instability," Phys. Rev. Lett., vol. 60, pp. 1278, 1988.

[16] D.H. Whittum et al, "Electron-hose instability in the ion-focused regime," Phys. Rev. Lett., vol. 67, pp. 991, 1991.

[17] A.A. Geraci, D.H. Wittum, "Transverse dynamics of a relativistic electron beam in an underdense plasma channel," Phys. Plasmas, vol. 7, pp. 3431, 2000.

[18] D.H. Whittum et al, "Flute instability of an ion-focused slab electron beam in a broad plasma," Phys. Rev. A, vol. 46, pp. 6684, 1992.

[19] S. Lee, private communication, 2000.

[20] P. Muggli et al, "Photo-ionized Lithium source for plasma accelerator applications," IEEE Transactions on Plasma Science, vol. 27, pp. 791, 1999.

[21] V.A. Lebedev, "Diffraction-limited resolution of the optical transition monitor,” Nucl. Instr. And Meth. A, vol. 372, pp. 344, 1996.

[22] M. Castellano and V.A. Verzilov, "Spatial resolution in optical transition beam diagnostics," Phys. Rev. ST Accel. Beams, vol 1, 062801, 1998. 
[23] S. Smith, P. Tenenbaum, S.H. Williams, "Performance of the beam position monitor system of the Final Focus Test Beam," Nuclear Instruments \& Methods in Physics Research, Section A, vol.431, pp.9, 1999.

[24] M.J. Hogan et al, "E-157: A 1.4-meter-long plasma wakefield acceleration experiment using a 30-GeV electron beam from the Stanford Linear Accelerator Center linac," SLAC-PUB-8352, Apr 2000.

[25] P. Muggli et al, "Lithium plasma source for acceleration and focusing of ultrarelativistic electron beams," Proceedings of the 1999 Particle Accelerator Conference, pp. 3651, 1999.

[26] J.D. Jackson, Classical Electrodynamics, $3^{\text {rd }}$ Ed. pp. 646-654, New York, John Wiley \& Sons, Inc., 1998.

[27] C. Clayton et al, in preparation.

[28] E. Dodd, submitted for publication.

[29] M.J. Hogan et at, "E162: Positron and electron dynamics in a plasma wakefield accelerator," SLAC-ARDB-242, Oct. 2000. 